\title{
Identification and characterization of molecular
}

mechanisms driving the functional specification of motor neurons

\section{The Delta like homolog 1 protein}

\author{
Dissertation \\ for the award of the degree \\ "Doctor of Philosophy” (Ph.D.) \\ Division of Mathematics and Natural Sciences \\ of the Georg-August-Universität Göttingen \\ submitted by
}

Daniel Müller

from

Heilbronn

Göttingen 2011 
Prof. Dr. Tomas Pieler (Reviewer)

Department for Developmental Biochemistry University of Göttingen

Prof. Dr. Andreas Wodarz (Reviewer)

Department of Stem Cell Biology

University of Göttingen

Dr. Till Marquardt (Supervisor)

Developmental Neurobiology

European Neuroscience Institute Göttingen (ENI-G)

Date of the oral examination: to be determined 


\section{Index of contents}

1. Abstract

2. Introduction 2

2.1 Motor neurons 2

2.1.1 Motor neuron development

2.2 Organization pattern of spinal motor neurons 4

2.2.1 Motor columns

2.2.2 The motor pools and their muscles

2.2.3 The motor neuron classes

2.2.4 Muscle fibers and their functional motor neuron types

2.2.4.1 Muscle fiber diversity

2.2.4.2 The functional motor neuron types 9

2.3 Motor neuron pathogenesis 11

2.3.1 Amyotrophic lateral sclerosis 12

2.4 The Delta-like homolog 1 protein (Dlk1) 13

2.4.1 The genomic localization the Dlkl gene 13

2.4.2 Dlk1 Protein structure 13

$\begin{array}{ll}\text { 2.4.3 Polar overdominance and imprinting } & 14\end{array}$

2.4.4 Putative Dlk1 functions in health and disease $\quad 15$

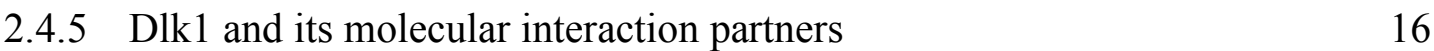

2.4.6 Dlk1 in the central nervous system 18

2.5 Aims of this work 19

3. Materials 20

3.1 Consumables and plastic ware 20

3.2 Antibodies

3.2.1 Primary antibodies 20

3.2.2 Secondary Antibodies 20

3.3. Mouse lines $\quad 20$

3.3.1 Hb9-GFP transgenic mice $\quad 20$

3.3.2 Dlkl knockout mice 21

$\begin{array}{lll}\text { 3.3.3 CD-1 Mice } & 21\end{array}$

3.4 Chemicals \& Reagents 21

3.5 Enzymes 23

3.6 Solutions 23

3.7 Kits 24

3.8 Software 24

3.9 Vectors $\quad 24$

4. Methods

4.1 Animal experiments in mice $\quad 25$

4.1.1 Mouse genotyping 25

4.1.1.1 DNA extraction from mouse tissue $\quad 25$

4.1.1.2 Genotyping of Hb9-GFP animals 26

4.1.1.3 Genotyping of $D l k l$ knockout animals $\quad 27$

4.1.2 Retrograde labeling experiments $\quad 27$

4.1.2.1 Retrograde tracing of motor neuron with cholera toxin B 27 
4.1.2.2 Isolation of retrogradely traced motor pools $\quad 27$

$\begin{array}{lll}\text { 4.1.3 Mouse behavioral experiments } & 27\end{array}$

$\begin{array}{ll}\text { 4.1.3.1 Grip strength assay } & 27\end{array}$

4.1.3.2 Running endurance test 28

4.1.3.3 Clasping assay 28

4.2 Quantitative expression analysis 29

4.2.1 RNA purification from microdissected motor pools 29

$\begin{array}{ll}\text { 4.2.2 cDNA synthesis for real-time experiments } & 29\end{array}$

$\begin{array}{ll}\text { 4.2.3 Quantitative real-time PCR } & 30\end{array}$

4.2.4 Synthesis of biotin-labeled aRNA for microarray hybridization 30

$\begin{array}{lll}\text { 4.2.5 Microarray hybridization } & 31\end{array}$

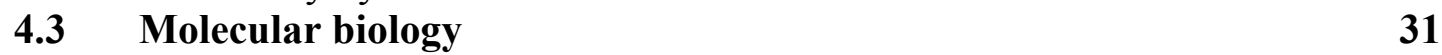

4.3.1 Preparation of chemically competent E.coli cells 31

4.3.2 Transformation of chemically competent E.coli 31

4.3.3 Cryopreservation and recovery of E.coli clones 31

$\begin{array}{lll}4.3 .4 & \text { Plasmid isolation } & 32\end{array}$

$\begin{array}{lll}\text { 4.3.5 DNA restriction digest } & 32 \\ \text { 4.3.6 } & \text { Alkatine phosphatase treanent }\end{array}$

4.3.6 Alkaline phosphatase treatment $\quad 32$

$\begin{array}{lll}\text { 4.3.7 } & \text { Agarose gel electrophoresis } & 32\end{array}$

4.3.8 Purification of DNA from agarose gels 33

4.3.9 Determination of nucleic acid concentration 33

4.3.10 DNA ligation 33

$\begin{array}{lll}4.4 & \text { Immunohistochemistry } & 33\end{array}$

4.4.1 Cryosectioning of mouse spinal cords and embryos 33

$\begin{array}{ll}\text { 4.4.2 Cryosectioning of chick embryo spinal cords } & 34\end{array}$

$\begin{array}{lll}\text { 4.4.3 Immunohistological staining procedure } & 34\end{array}$

$\begin{array}{lll}\text { 4.5 In-situ hybridization } & \mathbf{3 4}\end{array}$

4.5.1 In-situ probe generation 34

4.5.2 Sample preparation for in-situ analysis of postnatal mouse tissue 35

4.5.3 Sample preparation for in-situ analysis of prenatal mouse tissue 35

4.5.4 In-situ probe hybridization and detection for postnatal mouse tissue $\quad 35$

4.5.5 In-situ probe hybridization and detection for prenatal mouse tissue $\quad 36$

$\begin{array}{lll}4.6 & \text { Imaging and image processing } & 37\end{array}$

$\begin{array}{lll}\text { 4.6.1 Confocal imaging } & 37\end{array}$

$\begin{array}{ll}\text { 4.6.2 Brightfield and fluorescence imaging } & 37\end{array}$

$\begin{array}{ll}\text { 4.6.3 Motor neuron size determination } & 37\end{array}$

$\begin{array}{lll}4.7 & \text { Chick embryo electroporation } & 38\end{array}$

4.8 The long-term expression vector system 38

$\begin{array}{lll}4.9 & \text { Electrophysiology } & 40\end{array}$

$\begin{array}{ll}4.10 & \text { Statistical analysis } \\ & 40\end{array}$

5. Results 41

5.1 Screening FMNT populations 41

5.2 Quantitative Expression analysis of FMNT populations 45

$\begin{array}{lll}\text { 5.2.1 Microarray analysis } & 45\end{array}$

5.2.2 Data verification by quantitative real-time PCR 47

$\begin{array}{lll}\text { 5.3 Qualitative Dlk1 expression analysis } & \mathbf{4 8}\end{array}$

5.3.1 Presence of Dlk1 expression at different rostrocaudal levels 48

$\begin{array}{ll}\text { 5.3.2 Dlk1 expression in different motor columns } & 50\end{array}$

5.3.3 Analysis of Dlk1 expression in alpha and gamma motor neurons 50 
5.3.4 Characterization of Dlk1 expression in different motor pools 52

5.4 Cell soma size as a marker for different motor neuron types $\mathbf{5 5}$

5.4.1 Size determination of alpha and gamma motor neurons at P4 55

5.4.2 Determination of cell size in different motor pools 57

5.4.3 Cell soma size distribution of Dlk1 expressing cells 59

5.4.4 Cell soma size distribution of Dlk1 expressing cells in

$\begin{array}{lll}5.5 & \text { Dlk1 expression during development } & 63\end{array}$

5.5.1 Dlk1 expression in the developing mouse embryo 64

5.5.2 Correlation of embryonic Dlk1 expression to the motor neuron markers 66

5.5.3 Expression of Dlk1 in different postnatal stages 68

5.6 Analysis of Dlk1 knockout mice

5.6.1 Genotyping and verification of the Dlkl knockout mice 69

5.6.2 Characterization of Dlkl knockout animals 69

5.6.2.1 The clasping phenotype in Dlkl knockout mice 70

$\begin{array}{ll}\text { 5.6.2.2 Running endurance test } & 72\end{array}$

$\begin{array}{ll}\text { 5.6.2.3 Grip strength assay } & 73\end{array}$

5.6.3 Effects of Dlkl loss in motor neuron gene signature $\quad 74$

$\begin{array}{ll}\text { 5.6.3.1 Gene expression analysis of } D l k l^{-/} \text {motor neurons } & 75\end{array}$

$\begin{array}{ll}\text { 5.6.3.2 Notch pathway gene expression } & 81\end{array}$

5.7 Long-term transgene expression in the chick embryo spinal cord 82

5.8 Effects of Dlk1 on the electrophysiological properties of chicken motor neurons $\quad 85$

$\begin{array}{lr}\text { 6. Discussion } & 88\end{array}$

6.1 General considerations regarding the differential
analysis of FMNT populations

6.2 Towards the identification of further functional motor
neuron type specific genes

6.3 The expression analysis of the Dlk1 protein in motor
neurons and other tissues

6.4 The role of Dlk1 during motor neuron development 93

6.5 Behavioral observations in the Dlk1 knockout mouse model 95

6.6 The effects and mechanisms of Dlk1 at a molecular level 97

6.7 Effects of Dlk1 on electrophysiological motor neuron properties 99

$\begin{array}{ll}\text { 6.8 A possible role of Dlk1 in motor neuronal pathology } & 100\end{array}$

$\begin{array}{lll}6.9 & \text { Outlooks } & 100\end{array}$

$\begin{array}{lr}\text { 7. Summary } & 103\end{array}$

$\begin{array}{lr}\text { 8. Literature } & 104\end{array}$

$\begin{array}{lr}\text { 9. Publications } & 128\end{array}$

$\begin{array}{lr}\text { 10. Acknowledgements } & 129\end{array}$

$\begin{array}{ll}\text { 11. Résumé } & 130\end{array}$

$\begin{array}{ll}\text { 12. Declaration } & 131\end{array}$ 


\section{List of figures}

$\begin{array}{lll}\text { Figure } & \text { Page }\end{array}$

\section{Introduction}

2.1 ChAT Immunostaining of an adult mouse spinal cord 2

2.2. Neuronal cell fate in the ventral spinal cord is determined by Shh 3

2.3 Scheme of motor neuron organization 4

2.4 Spinal motor neurons are organized in four major motor columns 5

2.5 Position of motor columns at different rotrocaudal levels 5

2.6 Motor columns are formed by individual motor pools innervating distinct target muscles

2.7 Motor pools generally are consisting of motor neurons from all three different classes

2.8 The alpha motor neuron class can be subdivided into four functional motor neurons types (FMNT) $\quad 8$

$\begin{array}{ll}2.9 & \text { A generic motor pool with its associated muscle. } \\ 2.11\end{array}$

$\begin{array}{lll}2.10 & \text { Dlk1 protein structure } & 14\end{array}$

2.11 Putative molecular interaction pathways for Dlk1 function 17

\section{Materials}

3.1 Dlk1 knockout strategy 21

\section{Methods}

4.1 The long-term stable expression system 39

\section{Results}

5.1 Schematic of the Soleus and the Rectus femoris muscle and motor pool

5.2 Retrograde traced quadriceps motor pools 43

5.3 Screen of fast and slow FMNT populations 44

5.4 Expression analysis of slow and fast FMNT enriched motor pools 45

5.5 Comparision of expression levels of selected genes 46

5.6 Quantitative real-time PCR analysis of Neurotensin and Dlk1 expression

5.7 In-situ analysis of Dlk1 expression at different rostrocaudal levels at $\mathrm{P} 4$

5.8 Immunohistological analysis of Dlk1 expression at different rostrocaudal levels in $\mathrm{Hb}$ 9-GFP transgenic mice at P4 49

5.9 Dlk1 expression in LMC and MMC $\quad 50$

5.10 Dlk1 expression in alpha motor neurons at P4 51

5.11 Dlk1 expression in different motor pools 53

5.12 Percentage of Dlk1 positive retrogradely labeled cells in different
motor pools at P4

5.13 Percentage of Dlk1 positive retrogradely labeled cells in Soleus

5.14 Cell size distribution and average cell size of alpha and gamma motor neurons at P4 56

5.15 Comparison of cell soma sizes of different motor pools at P4 58

5.16 Cell size quantification of different motor neuron populations at $\mathrm{P} 4 \quad 60$ 
5.17 Size distribution of Dlk1 positive motor neurons in different motor pools at $\mathrm{P} 4$

5.18 Size distribution of Dlk1 positive motor neurons in the Soleus and Rectus femoris motor pool at P10

5.19 In-situ analysis of Dlk1 expression in the embryonic mouse spinal cord at different stages

5.20 In-situ hybridization for Dlk1 combined with anti-Isl1 antibody staining at E10.5 and E15.5

5.21 Dlk1 immunostaining at mouse spinals cord from different postnatal stages

5.22 Genotyping of Dlk1 knockout mice and verification of Dlk1 loss 69

5.23 The clasping phenotype in Dlkl knockout mice 70

5.24 Running endurance of $D l k l$ knockout and wild-type mice $\quad 72$

5.25 Grip strength measurement of Dlkl knockout and wild-type mice $\quad 74$

5.26 Heatmap of the genes upregulated in Dlkl wild-type Quadriceps MNs genes $\quad 76$

5.27 Heatmap of the genes upregulated in Dlkl knockout Quadriceps MNs $\quad 77$

5.28 Gene expression changes putatively linked to a systemic loss of Dlk1 function

5.29 Fast FMNT specific expression changes in $D l k 1^{-/-}$mice 79

5.30 Genes with switching expression in between Dlk1 wild type and knockout MNs

5.31 Expression analysis of Notch pathway genes in Dlk1 wild $\begin{array}{ll}\text { type and knockout MNs } & 81\end{array}$

5.32 GFP expression in motor neurons in chicken embryos at E18 83

5.33 Cell type specific long-term transgene expression GFP 84

5.34 Electrophysiological analysis of chick MNs overexpressing Dlk1 86

\section{Discussion}

6.1 Workflow for the identification of FMNT specific genes 90

6.2 The known molecular markers of motor neuron classes and types 92

6.3 The expression of Dlk1 in different stages of motor neuron development 95

$\begin{array}{ll}\text { 6.4 The bistable behavior of MNs is altered in Dlk1 knockout mice } & 101\end{array}$

6.5 Further studies to elucidate the role of Dlk1 in motor neuron development and energy metabolism 


\section{Table of symbols and abbreviations}

$\mu$

$\%$

A

e.g.

$\mathrm{F}$

ALS

aRNA

BSA

cDNA

ChAT

CNS

DEPC

DIG

Dlk1

DMSO

DNA

DSL

EGFP

Fig.

FMNT

$\mathrm{g}$

GFP

$\mathrm{h}$

HMC

Isl1

1

LMC

$\mathrm{LMCl}$

$\mathrm{LMCm}$

$\mathrm{m}$

M
Micro

Percent

Ampere

for example

Farad

Amyotrophic lateral sclerosis

Amplified RNA

Bovine serum albumin

Complementary DNA

Choline acetlytransferase

Central nervous system

Diethylpyrocarbonate

Digoxigenin

Delta like homolog 1

Dimethyl sulfoxid

Deoxyribonucleic acid

Delta/Serrate/lag-2

Enhanced green fluorescent protein

Figure

Functional motor neuron type

Gram

Green fluorescent protein

Hour

Hypaxial motor column

Islet 1

Liter

Lateral motor column

Lateral LMC

Medial LMC

Milli

Molar 


\begin{tabular}{|c|c|}
\hline $\min$ & Minute \\
\hline MMC & Medial motor column \\
\hline $\mathrm{MN}$ & Motor neuron \\
\hline mRNA & Messenger RNA \\
\hline NeuN & Neuronal nuclei \\
\hline NLS & Nuclear localization signal \\
\hline NMJ & Neuromuscular junction \\
\hline Nts & Neurotensin \\
\hline OCT & Optimal Cutting Temperature \\
\hline o.n. & Over night \\
\hline $\mathrm{p}$ & Pico \\
\hline PBS & Phosphate buffered saline \\
\hline PCR & Polymerase chain reaction \\
\hline PGC & Pregenglionic chain \\
\hline $\mathrm{pMN}$ & Motor neuron progenitors \\
\hline RNA & Ribonucleic acid \\
\hline RF & Rectus femoris \\
\hline S & Soleus \\
\hline $\sec$ & Second \\
\hline Shh & Sonic hedgehog \\
\hline TB & Triceps brachii \\
\hline $\mathrm{TC}$ & Tibialis cranialis \\
\hline vAChT & Vesicular acetylcholine transporter \\
\hline $\mathrm{w} / \mathrm{v}$ & Weight per volume \\
\hline $\mathrm{x} g$ & gravitational acceleration $(9.81 \mathrm{~m} / \mathrm{s})$ \\
\hline
\end{tabular}





\section{Abstract}

\section{Abstract}

Spinal Motor neurons are located in the grey matter of the ventrolateral spinal cord. They are playing a decisive role in body posture and locomotion by controlling skeletal muscle contraction via their motor axon termini. During embryonic development motor neurons arise from neural progenitor cells, move to their appropriate position and differentiate in order to innervate their proper muscle fibres. During this process, all motor neurons controlling a certain muscle organize in one distinct cluster termed 'motor pool'.

Some of the mechanisms responsible for the initial stages of motor neuron development, like motor column and motor pool formation have already been understood during the recent years (Dalla Torre di Sanguinetto 2008, Dasen et al. 2008). However, the molecular mechanisms underlying the final steps of motor neuron specification remained unknown so far. During these steps, motor neurons are specified into different functional motor neuron types (FMNT) in order to match their physiological properties with that of the different muscle fibre types they innervate. The different FMNTs furthermore exhibit varying vulnerability towards the neurodegenerative disease amyotrophic lateral sclerosis (ALS).

The aim of this work was to identify molecular markers for the different fast or slow motor neuron types and to elucidate their role in the formation and function of different FMNT populations. Therefore, a screen based on two motor neuron populations that were either almost entirely consisting of fast or slow motor neurons was designed and a microarray screen was performed in order to analyze the differential gene expression. As part of this screen the fast motor neuron specific gene Dlk1 was identified as a marker gene for fast motor neurons. The expression of Dlk1 throughout different motor neuron populations and developmental stages was verified. In order to reveal the functional role of Dlk1, knockout animals were behaviorally characterized and defined motor neuron populations of knockout and wild type were screened for differential gene expression by microarray analysis. 


\section{Introduction}

\section{Introduction}

\subsection{Motor neurons (MN)}

In order to conduct the complex voluntary and involuntary muscle contractions permanently required for proper locomotion and body posture, the muscle fibers rely on precisely orchestrated inputs from the central nervous system. These inputs are conducted by a specialized population of large soma size cells located in the ventral grey matter of the vertebrate spinal cord, termed spinal, or lower motor neurons $(\mathrm{MN})$. The motor neuron's function is to transmit electrical signals from the central nervous system (CNS) towards its associated muscle fiber. These signals are conducted as action potentials along the motor axons to the neuromuscular junction (NMJ), where they evoke release of synaptic vesicles containing the neurotransmitter acetylcholine, thus initiating muscle fiber contraction. The motor axons exit the spinal cord bundled in the ventral root, forming the peripheral nerves together with afferent sensory axons from the dorsal root ganglia (Honig et al. 1998). Amongst the signals motor neurons receive are efferent supraspinal and intraspinal inputs as well as 1a afferent inputs from sensory neurons. At the molecular level spinal motor neurons can be identified based on the expression of marker genes like the homeobox gene $\mathrm{Hb} 9$ (Hb9), the choline acetyltransferase (ChAT) gene or the vesicular acetylcholine transporter gene (vAChT) (Pfaff et al. 1996).
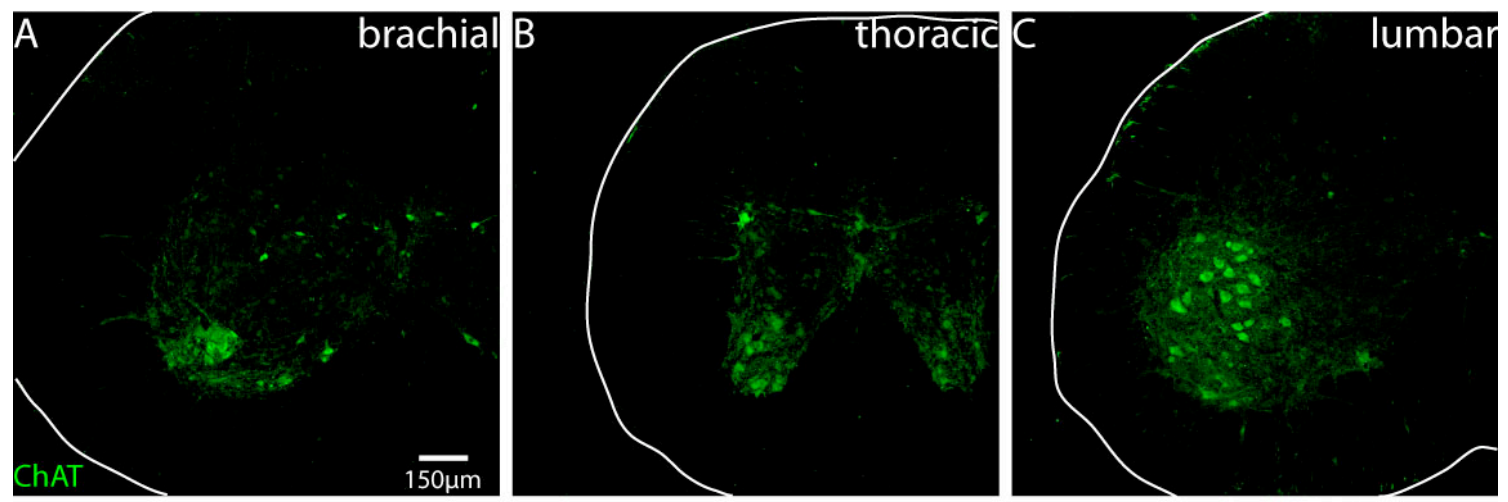

Fig. 2.1 ChAT Immunostaining of an adult mouse spinal cord. Expression of the choline acetyltransferase gene marks motor neurons and cholinergic interneurons at different rostrocaudal levels (brachial (A), thoracic (B) and lumbar (C)). The white line indicates the boundaries of the spinal cord. 


\section{Introduction}

\subsubsection{Motor neuron development}

During neurogenesis spinal neuron populations acquire different cell fates. These fates are determined as a response to exogenous signals. The most decisive signal in the development of ventral spinal cord neuronal populations is the sonic hedgehog gradient (Martì et al. 1995, Ericson et al. 1997). The sonic hedgehog protein is expressed in the notochord and the floor plate. After secretion it generates a ventral to dorsal concentration gradient. In response to that signal a certain population of neural tube progenitors termed motor neuron progenitors ( $\mathrm{pMNs}$ ) begin to express a subset of transcriptional regulators essential for the acquisition of the motor neuron cell fate (Briscoe \& Ericson 2001). The combinatorial action of these transcriptional regulators like Nkx 6.1, Nkx 6.2 and Pax6 (Briscoe et al. 2000, Cai et al. 2000, Vallstedt et al. 2001) promote the expression of the initial key determinant of $\mathrm{MN}$ fate, the Olig2 gene that is exclusively restricted to the pMNs (Novitch et al. 2000).

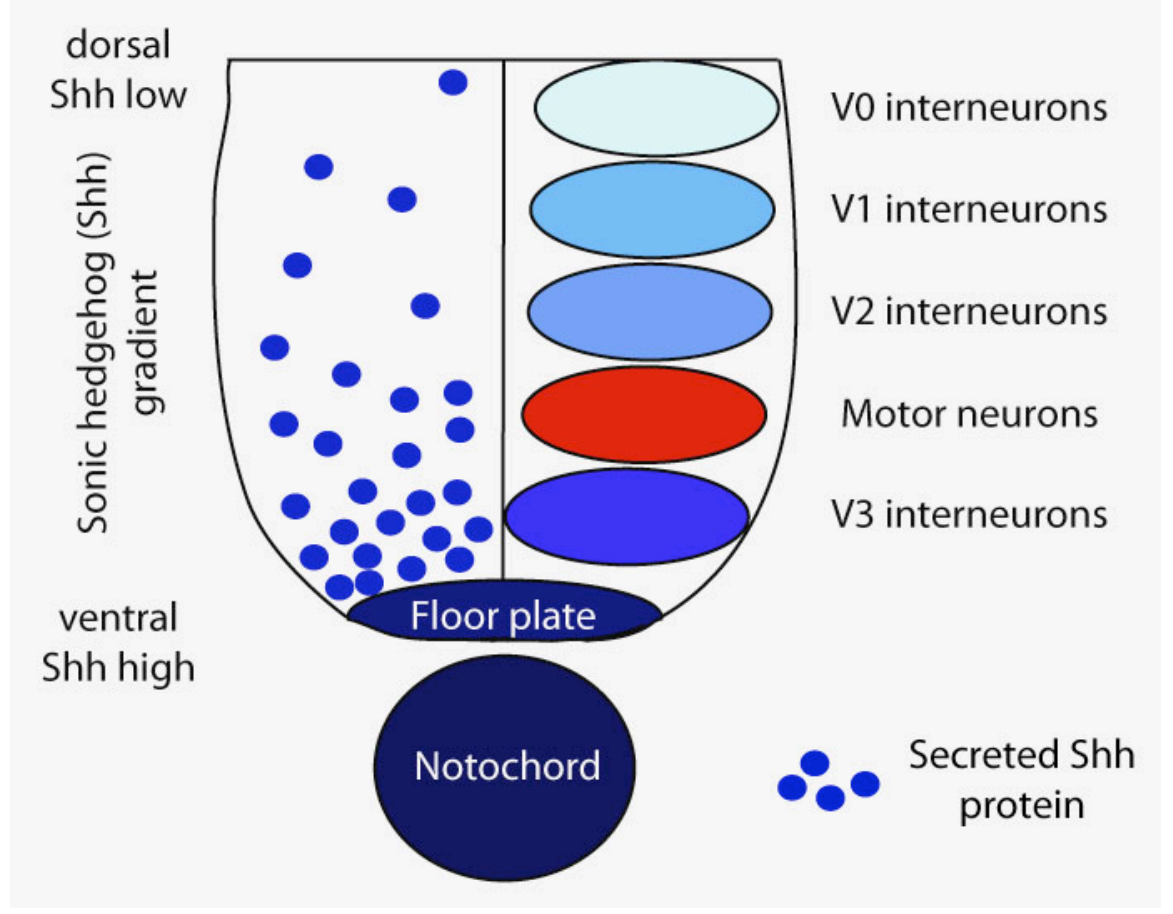

Fig. 2.2 Neuronal cell fate in the ventral spinal cord is determined by Shh. Notochord and floor plate are secreting sonic hedgehog (Shh) protein. Neuronal progenitors acquire their identity in response to the concentration of Shh they are subjected to. Modified after Jessell 2000 and Shirasaki \& Pfaff 2002.

Olig2 is a key factor in $\mathrm{MN}$ development, as it can evoke ectopic MN generation after overexpression in the spinal cord, however it is downregulated immediately in the newly generated MNs. Olig2 drives the expression of Neurogenin 2 (Ngn2) in the pMN cells, but inhibits expression of postmitotic MN specific genes like $\mathrm{Hb} 9$. 


\section{Introduction}

However, as Ngn2 accumulates in some of the pMNs it drives them from the progenitor state towards postmitotic MNs (Lee et al. 2005). Yet, the pMN domain remains active after an initial phase of $\mathrm{MN}$ generation, but switches to the generation of oligodendrocytes (Richardson et al. 2000, Lu et al. 2002).

A defining hallmark of spinal motor neuron identity is the expression of the $\mathrm{Hb} 9$ gene. This transcriptional regulator is specific for postmitotic MNs and suppresses the expression of interneuron specific genes in motor neurons, thereby consolidating MN cell fate (Thaler et al. 1999).

\subsection{Organization pattern of spinal motor neurons}

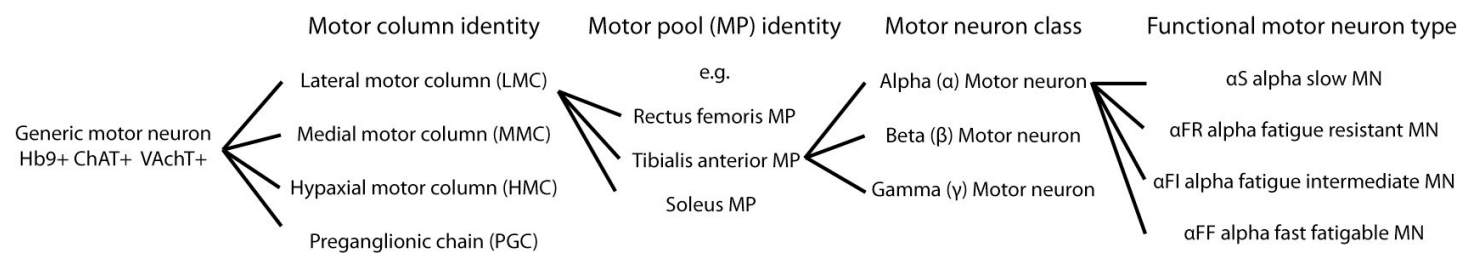

Fig. 2.3 Scheme of motor neuron organization. Each generic motor neuron can be defined by its motor column, its motor pool, its class and in case of alpha MNs by its functional motor neuron type.

Motor neurons are organized in a way reflecting the structure of the body musculature. The most paramount level of motor neuron organization is the motor column. A motor column contains all motor neurons innervating a large muscular structure like the limb or the body wall musculature. Each motor column consists of several motor pools innervating distinct target muscles. A motor pool is defined as the totality of all MNs innervating the same muscle. In an individual motor pool generally all classes of motor neurons (alpha, beta and gamma) can be detected. The alpha MNs can be further subdivided into four functional motor neuron types $(\alpha \mathrm{S}, \alpha \mathrm{FR}, \alpha \mathrm{FI}$ and $\alpha F F)$ innervating their distinct target muscle fiber types. 


\section{Introduction}

\subsubsection{Motor columns}

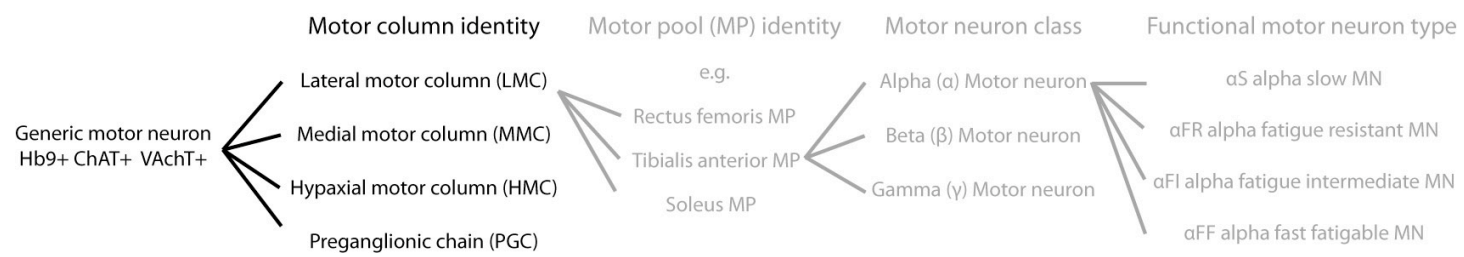

Fig. 2.4 Spinal motor neurons are organized in four major motor columns.

Four main motor columns can be distinguished within vertebrates. Each of these columns bears motor neurons stereotypically innervating a certain type of musculature. The medial motor column (MMC) contains the motor neurons innervating the axial musculature (Fetcho 1987, Gutman et al. 1993). The MMC is characterized by its presence along the whole rostrocaudal axis of the spinal cord, which contrasts the other motor columns. The lumbar motor columns (LMC) are only present at brachial and lumbar levels innervating limb musculature while the hypaxial motor column (HMC) and the preganglionic chain (PGC) innervating the body wall musculature or the sympathetic ganglia respectively are only present at thoracic levels (Fig. 2.5) (Dasen \& Jessell 2009).

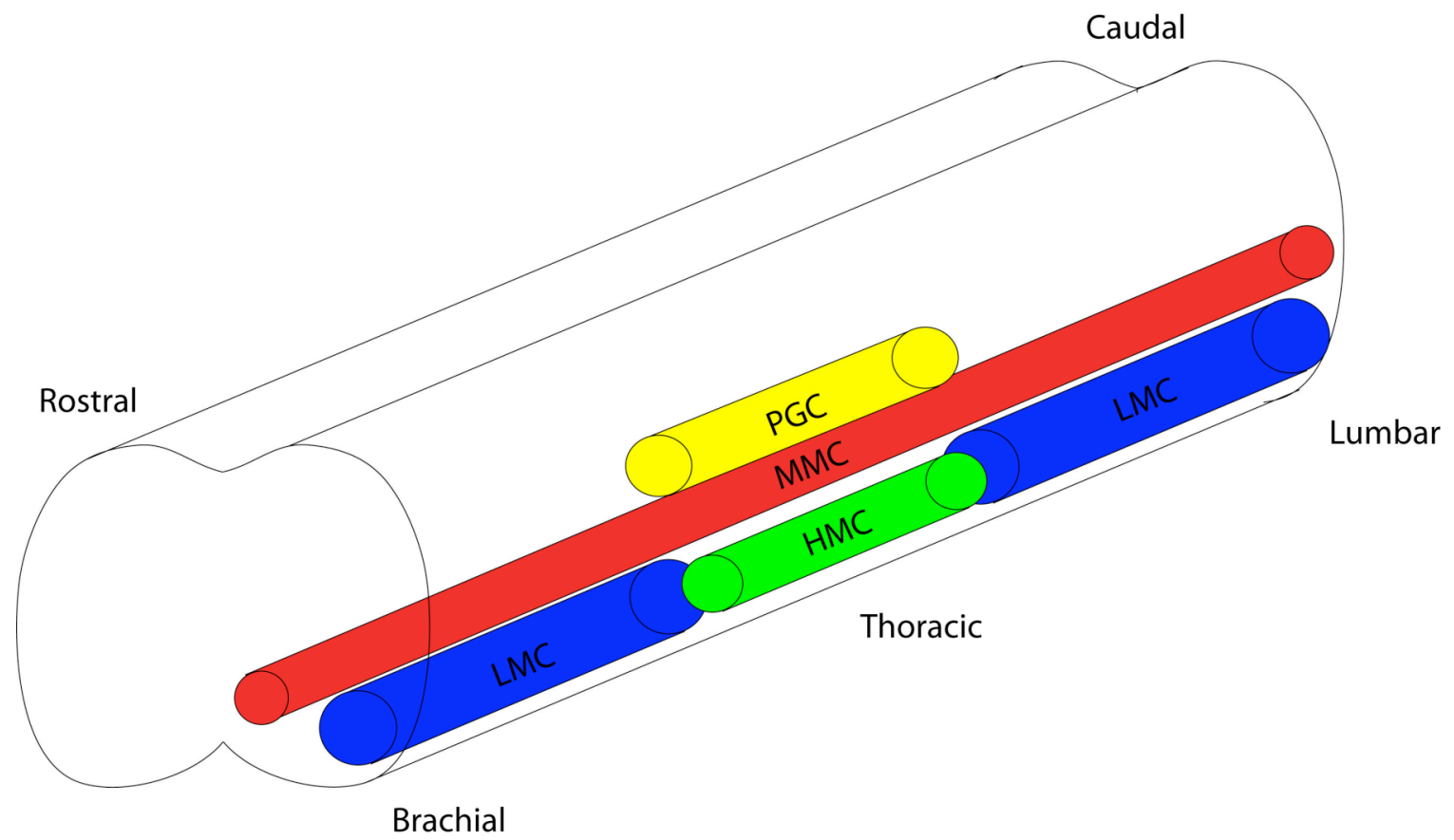

Fig. 2.5 Position of motor columns at different rotrocaudal levels. While medial motor column (MMC) MNs are present at all rostrocaudal levels, lateral motor column MNs are only found at the brachial and lumbar levels while preganglionic chain (PGC) MNs and hypaxial motor column (HMC) MNs can be found at the thoracic level. (Modified after Dasen et al. 2008 and Kanning et al. 2010) 


\section{Introduction}

During development motor neuron columnar identity is defined by the expression of distinct genetic marker genes. The brachial LMC is defined by the expression of the Hox6 paralogs, the lumbar LMC by Hox 10 paralogs and the PGC by Hox9 paralogs (Dasen et al. 2003, Shah et al. 2004, Dasen et al. 2008, Wu et al. 2008). The activity of these Hox genes is modulated via FoxP1 (Dasen et al. 2008). This is contrasted by the mechanism underlying MMC motor neuron identity that is expected to be promoted via Wnt4/5 signaling and is defined by expression of Lhx3 (Agalliu et al. 2009).

\subsubsection{The motor pools and their muscles}

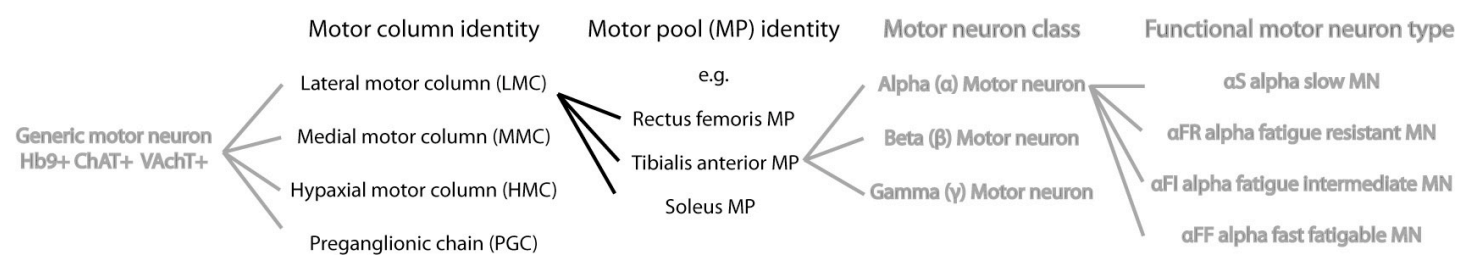

Fig. 2.6 Motor columns are formed by individual motor pools innervating distinct target muscles.

The motor neurons innervating a distinct muscle are organized in a cigar shaped cluster within the spinal cord and are termed "motor pool". Motor pools occupy a stereotypic position along the rostrocaudal axis. The relative position of a motor pool resembles the structure of the body musculature. Thus, motor pools are organized in a somatotopic manner. (Romanes 1951, Landmesser 1978, Vanderhorst \& Holstege 1997). The molecular identity of the different motor pools is defined by the expression of a distinct pattern of Hox and Lim transcription factors, therefore it is thinkable that each single motor pool can be identified by a distinct Hox pattern fingerprint at a molecular level (Dasen et al. 2005, Dalla Torre di Sanguinetto 2008). Within a motor pool cell-to-cell recognition is modulated via homophilic interaction of Cadherin class proteins (Price et al. 2002). Hence, it is likely that similar mechanisms and guidance cues are involved in recognition of the motor pools target muscles. 


\section{Introduction}

\subsubsection{The motor neuron classes}

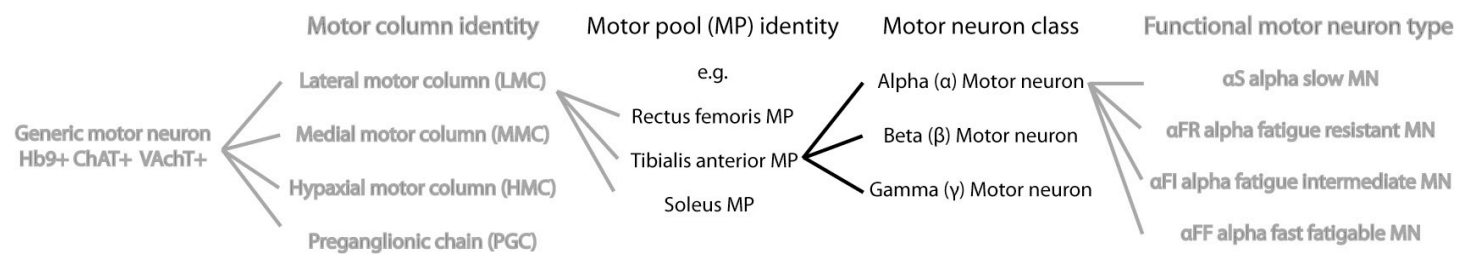

Fig. 2.7 Motor pools generally are consisting of motor neurons from all three different classes.

Three specialized classes of MNs have been identified so far, which are present at all rostrocaudal levels and in almost all motor pools throughout the spinal cord. While alpha MNs are connected to extrafusal fibers only, the gamma MNs exclusively innervate the intrafusal fibers of the muscle spindles. The third group of motor neurons are the beta MNs that are believed to innervate intra- and extrafusal fibers, however these cells have not been well characterized as yet (Banks 1994). The typical skeletal muscle motor pool includes MNs of all types. An exception is found in the facial motor pools, which seem to lack gamma MNs, as facial muscles do not possess muscle spindles (Bowden \& Mahran 1956, Whitehead et al. 2005). Alpha and gamma MNs have contrasting functional roles. Whereas alpha motor neurons are responsible for the generation of the muscle contraction by activating the extrafusal muscle fibers, the gamma MNs innervate the intrafusal fibers of the muscle spindles and hence play part in a sensory organ monitoring and adjusting muscle contraction (Hunt \& Kuffler 1951). The ratio of alpha MNs to gamma MNs is claimed to be 2 to 1 (Kuffler et al. 1951, Friese at al. 2009).

At the morphological level alpha MNs can be distinguished from gamma MNs by their cell soma size, which is significantly larger for alpha MNs (Burke et al. 1977, Friese et al. 2009, Shneider et al. 2009). Additionally, the axons of gamma MNs are thinner compared to alpha MN axons (Westbury 1982). These structural differences are reflected by discrete electrophysiological properties like the input resistance and the axonal conduction velocity. While the input resistance of gamma MNs is higher due to the smaller cell size, the axonal conduction velocity is lower due to the thinner axonal diameter in the cat model system (Kemm \& Westbury 1978). Recently Friese et al. and Shneider et al. have published a set of potential molecular markers for these MN classes in mice (Friese et al. 2009 and Shneider et al. 2009). While Friese and colleagues have found the orphan nuclear receptor estrogen related receptor 3 (Esrr3) 


\section{Introduction}

to be expressed exclusively in gamma MNs, Shneider et al. suggest the glia cell derived neurotrophic factor receptor $\alpha 1$ (Gfro1) to be specific to gamma MNs. In addition Friese et al. propose that the strong expression of the neuronal nuclei protein $(\mathrm{NeuN})$ is limited to alpha MNs.

Another remarkable difference between motor neurons of the alpha and gamma class is the preferential axonal uptake of tetanus toxin. According to a study in cat, gamma fibers do not take up tetanus toxin, while it is found to be transported within alpha $\mathrm{MN}$ axons (Green et al. 1977).

\subsubsection{Muscle fibers and their functional motor neuron types}

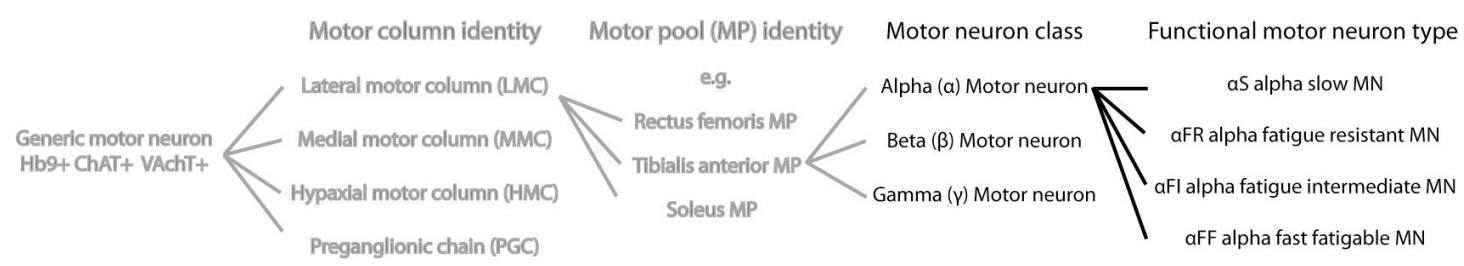

Fig. 2.8 The alpha motor neuron class can be subdivided into four functional motor neurons types (FMNT)

\subsubsection{Muscle fiber diversity}

A consideration of the functional motor neuron types would be incomplete without a prior look on their associated muscle fiber types. Therefore, an overview of the muscle fiber diversity is given here. Muscle fibers are differing in their functional properties reflected by variations in numerous parameters. Thus, one of the possibilities to classify muscle fibers is based on expression of the four different myosin heavy chain types (1, 2a, 2x and 2b) (Schiaffino et al. 1989, Schiaffino \& Reggiani 1994). Other key differences between the different muscle fiber types are the velocity of fiber twitch, the content in myoglobin, the fatigability and the primary source of energy utilized (Jansson \& Sylvèn 1983).

Most muscles resemble a mosaic of all four different types of extrafusal muscle fibers in varying proportions (Wigmore \& Evans 2002). The specific molecular and physiological profiles reflect the functional role of the different muscle fiber types. Whereas the type 1 fibers can provide a long lasting constant contraction and are initially contracting in the process of muscle activation, the fast twitch type $2 \mathrm{a}$, type $2 \mathrm{x}$ and type $2 \mathrm{~b}$ fibers are recruited gradually upon demand of higher muscular force 


\section{Introduction}

output. This sequential recruitment results in a smooth muscular force output that reaches the peak with contraction of the type $2 b$ fibers. However, the type $2 b$ fibers can maintain their contraction only for a very short period of time as they exhaust their cellular energy reserves more rapidly. Due to their metabolic and physiological properties the type $2 \mathrm{~b}$ are also referred as fast glycolytic muscle fibers. They do not possess large myoglobin reserves, as they use the glycolysis of glucose as almost exclusive source of energy. The lack of the muscular oxygen storage protein myoglobin is reflected by their pale pink color, hence the type $2 \mathrm{~b}$ fibers are often denoted as white muscle fibers. The properties of the slow-twich oxidative type 1 fibers contrast with that of the fast glycolytic fibers. Their energy supply is maintained primarily through oxidation of fatty acids. They express high levels of myoglobin as an oxygen buffer, and accordingly show a dark orange to red color. The properties of the other two muscle fiber types lie between these extremes.

Muscle fibers seem to rely on input by their specific FMNTs in order to maintain their functional properties. Since surgical experiments that result in ectopic innervation of a muscle with a nerve normally supporting a muscle with different muscle fiber composition are sufficient to evoke changes in the myosin expression profile (Fournier le Ray et al. 1989). A similar observation was made in muscles subjected to chronic electrical stimulation mimicking specific neuromuscular input by a distinct FMNT (Pette \& Vrbovà 1992, Bacou et al. 1996).

\subsubsection{The functional motor neuron types}

Different types of alpha MNs exist that precisely match the physiological and functional properties of the innervated muscle fiber types. Hence, the class of alpha MNs can be further subdivided. In this work four different muscle fiber types with four different associated FMNTs are assumed (Fig. 2.9). The number of four different FMNTs is debatable. It is based on the four different types of myosin heavy chain proteins described by Schiaffino et al., other authors however are claiming the presence of a lower number of FMNTs as the three fast motor types are not quiet clearly distinguishable (Schiaffino et al. 1989, Bakels \& Kernell 1993, Gardiner 1993). Nevertheless, it is known that one type of muscle fibers is receiving synaptic input only from one FMNT (Rafuse et al. 1996, Mantilla \& Sieck 2003).

The nomenclature for the different FMNTs is partially based on the innervated muscle fiber types. While the slow-twitch type 1 muscle fibers are innervated by MNs 


\section{Introduction}

denominated $\alpha \mathrm{S}$ or slow alpha MNs, the faster type 2a fibers are linked to the fatigue resistant or $\alpha F R$ MNs. The MNs termed $\alpha F I$ or fatigue intermediate are associated with the type $2 \mathrm{x}$ muscle fibers and finally the $\alpha \mathrm{FF}$ or the fast fatigable MNs innervate the type $2 \mathrm{~b}$ fibers (Fig. 2.9). These homogenous assemblies consisting of an individual motor neuron on the one side and all its innervated muscle fibers is termed as "motor unit", as all muscle fibers are acting concerted upon stimulation from the MN. The motor units therefore form the basic building blocks of neuromuscular function (Kernell 2003).

To date, characterization of the different FMNTs has been restricted primarily towards studying their morphological and physiological properties. Thereby several characteristics of FMNTs could be defined. On the morphological level fast FMNTs can be distinguished from slow ones by the cell size. Fast MNs are larger and possess thicker axons in cats and rodents (Webber \& Pleschka 1976, Burke et al. 1977, Kernell \& Zwaagstra, 1981, Ulfhake \& Kellerth 1982, Ishihara et al. 1988). Fast and slow neuromuscular synapses are also differing in their shape. While fast synapses exhibit the classical "pretzel" structure and appear to be broader and more complex, the slow ones are longer and appear to have a simpler structure (Sieck \& Prakash 1997). Furthermore fast motor units are bigger than small ones, as a single fast $\mathrm{MN}$ is innervating more muscle fibers compared to a slow MN (Burke \& Tsairis 1973).

Other well-understood differences lie within the eletrophysiological characteristics. Some properties like higher input resistance or the lower conduction velocity could directly be explained by the smaller cell soma size or the thinner axons of slow MNs (Zengel et al. 1985, Gardiner 1993, Bakels \& Kernel 1993). Other characteristics like the tonic firing pattern upon stimulation of slow MNs contrasting the phasic firing pattern of fast MNs are obvious due to the contractile characteristics of the innervated muscle fibers, although the molecular mechanisms underlying this observation are unclear (Lee \& Heckmann 1998). The same applies for the lower rheobase associated with slow MNs, as they are activated earlier during MN recruitment. Moreover slow MNs are exhibiting a more pronounced bistable behavior upon stimulation (Lee \& Heckmann 1998). Additionally, the different FMNTs exhibit a strong difference in vulnerability towards motor neurons diseases and aging (Hashizume et al. 1988, Kadhiresan et al. 1996, Pun et al. 2006) 


\section{Introduction}

Until recently, no molecular markers for different FMNTs have been available. Recently, a set of potential marker genes has been published. While Chakkalakal et al. suggest the synaptic vesicle protein 2a (SV2a) specifically expressed within adult stage slow motor neurons, Enjin et al. 2010 have found the Estrogen related receptor beta (Esrrb) protein in P0 and P11 slow motor neurons (Chakkalakal et al. 2009, Enjin et al. 2010). Enjin and colleagues are also proposing the fast motor neuron markers Chondrolectin (Chodl) and Calcitonin 1 (Calca) according to cell size correlation and differential expression towards Esrrb positive neurons. However, apart from SV2a, neither of these genes, has been throughoutly linked to fast or slow FMNTs. Moreover, their role in fast/slow MN development or function remains to be addressed.

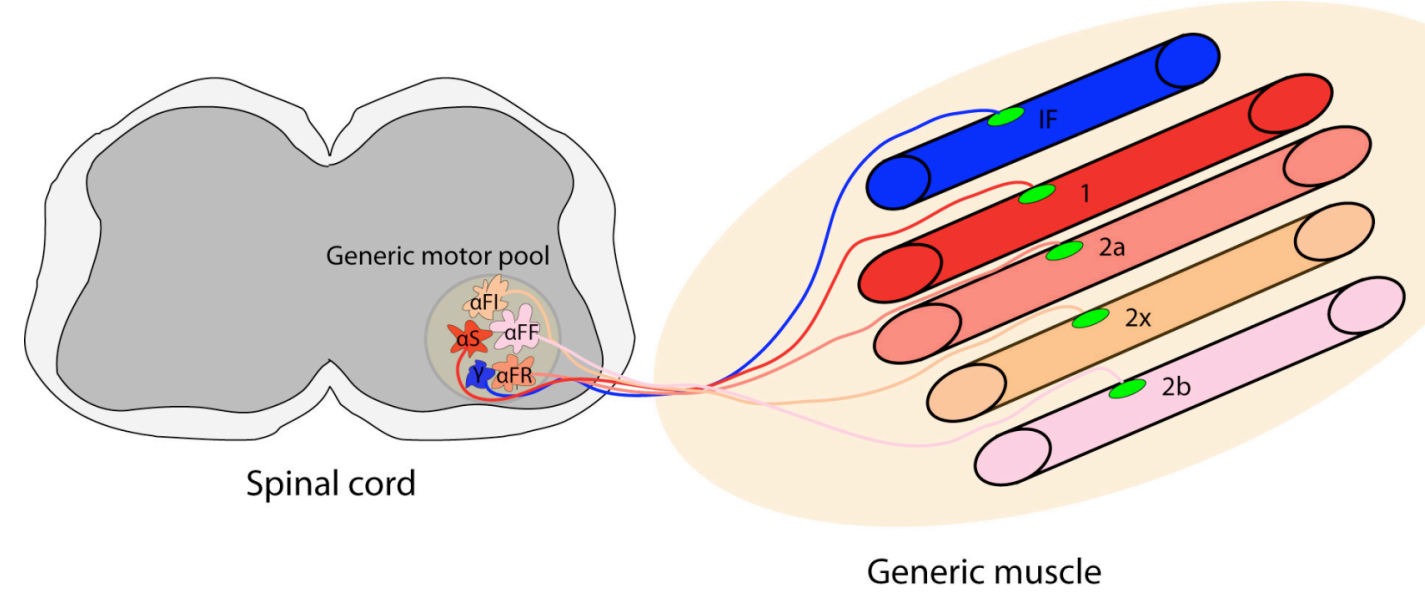

Fig. 2.9 A generic motor pool with its associated muscle. Motor neurons of a distinct FMNT are stereotypically innervating muscle fibers of their associated muscle fiber type in a target muscle. The motor pools FMNT composition therefore is correlated with the muscle fiber composition of the target muscle.

\subsection{Motor neuron pathogenesis}

Loss of motor neurons or their inability to evoke muscle contraction is associated with several diseases and pathogenic states. Some of them are leading to an acute failure of motor neuron function others are gradually advancing over a time period of several years. A common aspect of all these pathogenic states is severe health impairment in the locomotor system, which can cumulate in death by respiratory arrest due to functional failure of the diaphragm. As an example, amyotrophic lateral sclerosis, one of the most well-known and intensively studied motor neuron diseases will be introduced in the following section. 


\section{Introduction}

\subsubsection{Amyotrophic lateral sclerosis}

Amyotrophic lateral sclerosis (ALS) in Northern America also referred as Motor Neuron Disease, or Lou Gehrig's disease is characterized by progressive loss motor neurons and associated loss of muscle function.

Only for a subset of ALS cases distinct genetic causes have been identified. Common etiological factors in a part of the inherited cases are mutations within superoxid dismutase 1 (SOD1) gene (Rosen et al. 1993). Furthermore, in the recent years new genetic factors for familial ALS could be defined like mutations in the TAR-DNA binding protein 43 or the FUS/TLS gene (Sreedharan et al. 2008, Kwiatkowski et al. 2009). However, most ALS cases seem to appear sporadically without a distinct known cause or common denominator (Valdmanis \& Rouleau 2008). Although a significant increase in occurrence of ALS cases was found in soccer players (Chiò et al. 2002, Chiò et al. 2005, Wicks et al. 2007).

ALS is of specific interest for this work as prior studies have suggested a partial resistance of slower motor units towards the disease (Frey et al. 2000, Pun et al. 2006, Hegedus et al. 2007). During disease progression in the familial ALS mouse model the FF MNs degenerate first leaving the fast muscle fibers deinnervated. This animal model transgenetically expresses a mutated form of the superoxide dismutase 1 gene (SOD1 G93A) that was initially found in human patients suffering from a familial form of ALS (Rosen et al. 1993, Guerney et al. 1994). These studies indicate that the different muscle fibers die in waves suggesting the same for the motor neurons. These results in the mouse model are consistent with observations in human patients, where the force output of the fast twitch motor units is lost earlier in the disease (Dengler et al. 1990). Remarkably certain populations of motor neurons remain intact even in patients with terminal stage ALS. These are the oculomotor neurons (Gizzi et al. 1992, Kaminski et al. 2002) and the Onuf's nucleus (Carvalho et al. 1995, Mannen 2000) motor neurons innervating the external sphincter muscles of the urethra and anus. Moreover the motor neurons forming the Onuf's nucleus seem to be unaffected not only in ALS, but also in Polio patients (Kojima et al. 1989). 


\section{Introduction}

\subsection{The Delta-like homolog 1 protein (Dlk1)}

In the frame of this work, the Delta-like homolog 1 was one of the genes identified in the initial screen for fast and slow motor neuron type specific marker genes. It was overexpressed in the predominantly fast FMNT containing Rectus femoris motor pool. As one of the most promising candidate genes Dlk1 was investigated within the framework of this thesis. Therefore a short overview about the present state of research regarding this gene is given.

The Delta-like homolog 1 (Dlk1) protein was initially found in human amniotic fluid and described as fetal antigen 1 (FA1) in (Fay et al. 1988). Later it was also discovered in lung cell carcinomas, neuroendocrine tumors and 3T3 mouse fibroblasts under the name delta-like and abbreviated dlk due to its high amino acid sequence homology to proteins of the Drosophila melanogaster Delta protein family (Laborda et al. 1993). As the actual homologs of the D. melanogaster Delta were discovered in vertebrates and denoted Delta-like (D11) the previously discovered protein is now referred to delta-like homolog 1, while the abbreviation Dlk1 is kept (Bettenhausen et al. 1995, Chitins et al. 1995, Henrique et al. 1995).

Dlk1 was rediscovered several times in different tissues or cell lines independently and therefore was given different denominations. The most common ones besides Dlk1 are Pref-1 for preadipocyte factor 1 or ZOG for zona glomerolosa-specific protein in rats (Smas \& Sul 1993, Okamoto et al. 1998).

\subsubsection{The genomic localization the Dlk1 gene}

The murine $D l k 1$ gene could be mapped to the chromosome 12 while in human it is located at chromosome 14 and chromosome 18 in sheep (Gubina et al. 1999, Fahrenkrug et al. 2000, Gubina et al. 2000). The gene is part of the genomic $D l k 1 / G t l 2$ locus that is imprinted in mammals (Schmidt et al. 2000, Wylie et al. 2000).

\subsubsection{Dlk1 Protein structure}

Dlk1 is posttranscriptional modified by alternative splicing, creating several protein isoforms (Fig. 2.10). All isoforms share a signal peptide for the secretory pathway, six epidermal growth factor (EGF) like repeats, a transmembrane region and a short cytoplasmatic C-terminus. The two N-terminal EGF-like repeats were also referred as 


\section{Introduction}

DOS (Delta or OSM-11)-motif (Komatsu et al. 2008). The main differences between the Dlk1 isoforms lie within the presence and size of the juxtamembrane region and the presence of the proximal tumor necrosis factor alpha converting enzyme (TACE) cleavage site (Wang \& Sul 2006). At this site Dlk1 is supposed to be cleaved into two parts releasing the supposedly active extracellular region with its six EGF-like repeats (Smas et al. 1997, Wang \& Sul 2006, Wang et al. 2006). The function of the different Dlk1 forms is disputed, since the short extracellular form generated from the distal cleavage site is not able to suppress fibroblast differentiation in adipocytes (Mei et al. 2002).

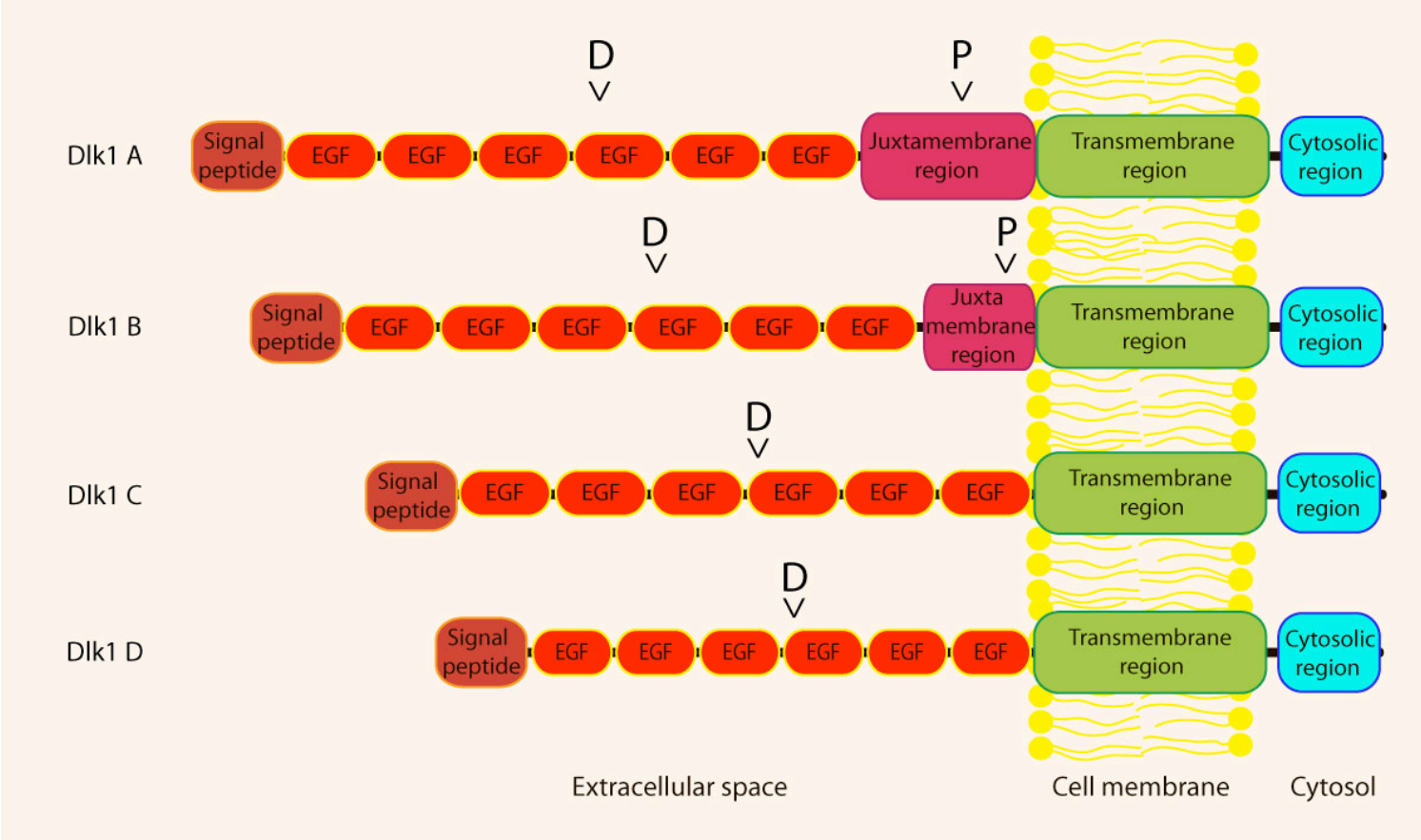

Fig. 2.10 Dlk1 protein structure Four known isoforms of Dlk1 are shown, common features of all isoforms are the six EGF-like repeats, the transmembrane region and the short cytosolic region. The proximal (P) and distal (D) TACE cleavage sites are marked. Modified after Wang et al. 2006.

\subsubsection{Polar overdominance and imprinting}

One of the most commonly discussed features of the Dlkl gene is its specific form of regulation by genetic imprinting. The gene is further associated with a specific form of muscular hypertrophy in sheep known as callipyge phenotype (Davis 2004, Murphy et al. 2005). This phenotype was initially discovered in the 1980s in sheep that exhibited remarkably large hindquarters with increased muscle but reduced fat content. Notably, the phenotype was not inherited according to a classical Medelian ratio. Research was limited in the beginning due to the lack of available founder 


\section{Introduction}

animals and the slow reproductive cycle of sheep compared to classical laboratory animals like rodents, but finally the callipyge inheritance was elucidated. Only animals that have gained the callipyge allele from their fathers showed the callipyge phenotype irrespective of the maternal allele. This phenomenon of paternal imprinting was denominated "polar overdominance" (Cockett et al. 1996).

\subsubsection{Putative Dlk1 functions in health and disease}

To date Dlk1 has been associated with several different functional roles in a wide range of organs and cell lines. The best understood function so far is the inhibition of the adipocyte differentiation in preadipocyte cell lines. To fulfill this function posttranslational cleavage by TACE at the proximal cleavage site to release a large soluble extracellular fragment appears to be decisive (Smas et al. 1997, Mei et al. 2002). While Dlk1 is normally highly expressed in the developing fetal muscles and at a lower level in postnatal muscle satellite cells a naturally occurring mutation, which leads to the postnatal overexpression of Dlk1 in sheep hindlimb muscles has been associated with increased muscle but reduced fat mass (Floridon et al. 2000, White et al. 2008). This would be consistent with the observation of Dlk1 reducing, but not entirely inhibiting fat cell development upon overexpression in mouse adipose tissue (Lee et al. 2003, Villena et al. 2008). The role of Dlk1 in muscle development was under investigation with special consideration of the callipyge phenotype in sheep and it is suggested to be involved in muscle regeneration. Conditional loss of Dlk1 in muscles moreover reduces the number of muscle fibers in general (Waddell et al. 2010). Dlk1 plays also a role in the immune system as it has an effect on B cell maturation and as a pro-inflammatory factor (Raghunanden et al. 2008, Chacón et al. 2008).

Since Dlk1 is found to be expressed in several cancers including neuroendocrine tumors and glioblastomas, and also increases malignancy upon overexpression in glioblastoma multiforme cell lines (Astuti et al. 2005, Yin et al. 2006, Yanai et al. 2010). Thus, a role as a putative tumor suppressor has been discussed (Kawakami et al. 2006, Espina et al. 2009). Due to partial overlap in symptoms between the phenotype of Dlk1 knockout mice and the human disease determined maternal uniparental disomy 14 observed by Moon and colleagues, Dlk1 has been implicated to contribute to this genetic disease (Moon et al. 2002, Falk et al. 2005). 


\section{Introduction}

\subsubsection{Dlk1 and its molecular interaction partners}

Based on its similarity to the Delta protein the most obvious mode of action postulated for Dlk1 was the prominent canonical Delta/Notch signaling pathway. However, the interaction between Notch proteins and Dlk1 remains controversial up to this point. Despite Dlk1 lacks the canonical DSL domain required for Notch activation signaling there is evidence on for an interaction on the protein level according to yeast-two hybrid assays (Tax et al. 1994, Baladron et al. 2005, Gordon et al. 2009). According to the results of Baladron et al. Dlk1 expression or addition of Dlk1 to the cell culture medium is able to decrease Notch signaling activity and downstream expression of the Notch target gene hairy and enhancer of split (Hes1). Also reduced Dlk1 expression in a knock down experiment was followed by an increase in Hes1 expression. Moreover Hes1 overexpression in 3T3 cells was shown to cause reduction in Dlk1 expression levels, indicating a kind of feedback inhibition mechanism between these genes (Ross et al. 2004). In Drosophila another observation was made that suggests Dlk1/Notch interaction, as expression of Dlk1 was able to reduce downstream gene expression in the Notch pathway and thereby exhibiting phenotypes similar to notch inactivation phenotypes in the Drosophila wing (Bray et al. 2008). Additionally, a recent study in conditional knockout mice lacking Dlk1 expression in skeletal muscles has shown that absence of Dlk1 leads to impaired muscle regeneration, which is suggested to be due to lack of myoblast differentiation caused by excess Notch activation (Waddell et al. 2010). Though these reports are proposing an inhibitory role for Dlk1 reducing Notch signaling a study in the nematode Caenorhabditis elegans suggests the contrary (Komatsu et al. 2008). Osm-11 the $C$. elegans homolog of Dlk1 is indicated to activate Lin-12, a Notch homolog in the same organism. Expression of mammalian Dlk1 within nematodes deficient for Osm-11 was able to rescue defects in vulval development claimed to be caused by impaired Lin-12 signaling. Also the results from Kaneta et al. are inconsistent with a Notch inhibitory role of Dlk1 as an increase of Hes1 expression in thymocytes after cultivation in Dlk1-Fc treated culture medium was observed, suggesting an enhanced Notch signaling activity (Kaneta et al. 2000). On the other hand several studies question involvement of Dlk1 in the Notch/Delta pathway in general. Instead an interaction of the Dlk1 juxtamembrane region with the insulin-like growth factor binding protein 1 (IGFBP1) was described (Nueda et al. 2008). Also the 


\section{Introduction}

extracellular signal-regulated kinase/mitogen activated protein kinase pathway is discussed to mediate the anti-adipogenic activity of Dlk1 (Kim et al. 2007). This signaling pathway is supposed to be activated via interaction of Dlk1 with fibronectin and subsequent downstream integrin signaling (Pankow \& Yamada 2002, Harburger \& Calderwood 2009, Wang et al. 2010). An overview of suggested and putative signaling mechanisms and interactions proposed for the protein is given in Figure 2.11 .

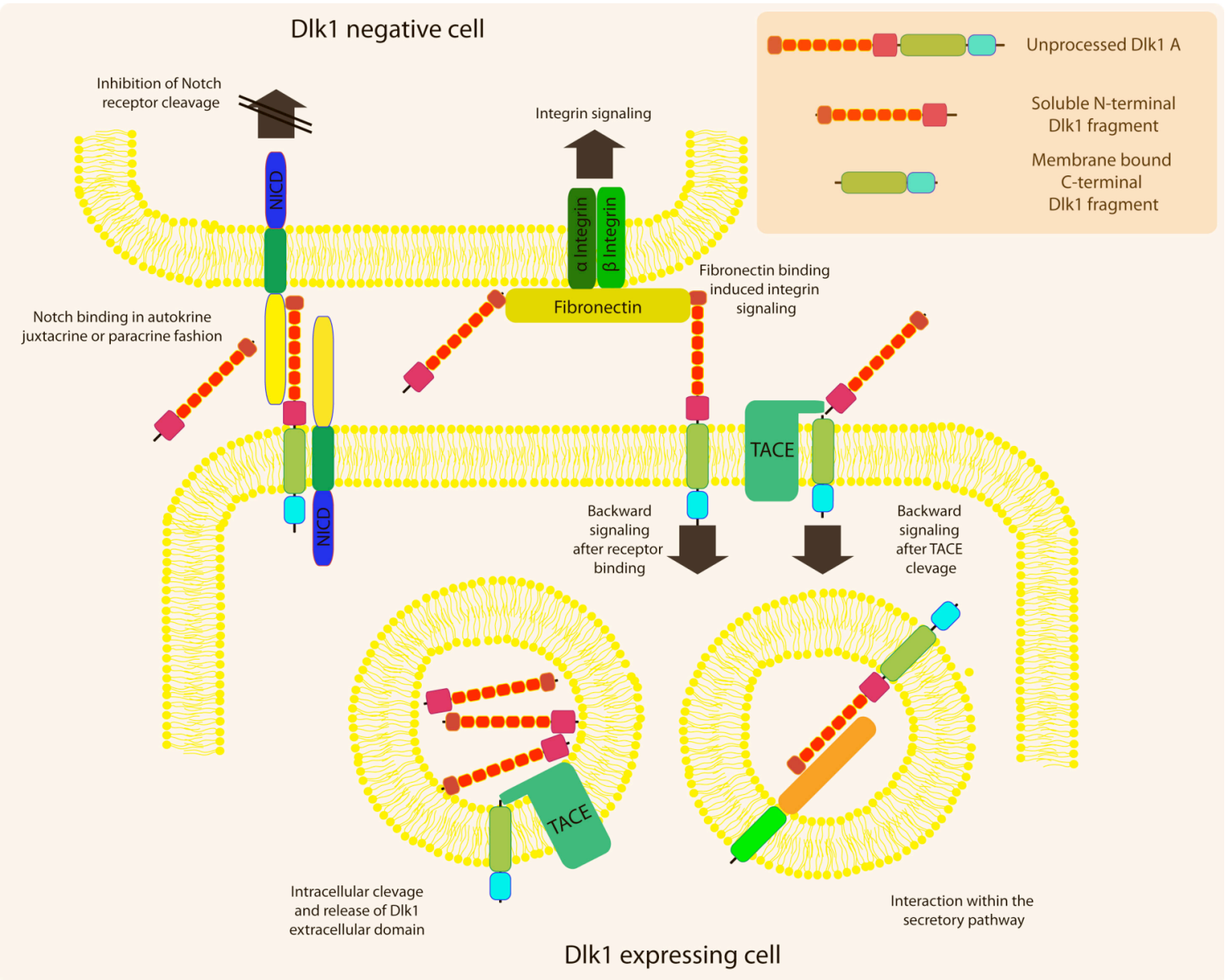

Fig. 2.11 Putative molecular interaction pathways for Dlk1 function. Dlk1 is claimed to interact with Notch or fibronectin and thereby influencing cell differentiation. While cleavage of Dlk1 by TACE is described it is unclear if this cut occurs in the secretory pathway or at the cell membrane. Although there is evidence for the interactions between Dlk1 extracellular domain and Notch1 or fibronectin, it is unclear if there are any cell autonomous interactions in the secretion pathway or by the cleaved C-terminus of the protein. 


\section{Introduction}

\subsubsection{Dlk1 in the central nervous system}

Dlk1 expression and functions have been characterized mainly in 3T3 preadipocytes and muscles so far. The reason for this likely lies with the easy access to the Dlk1 functional pathway in 3T3-L1 cells, which are directly responding towards changes in the Dlk1 pathway by a drastic phenotypic shift. The analysis of Dlk1 gene function in muscles was moreover accelerated by the economic relevance of the callipyge phenotype in sheep. However, the role Dlk1 is playing in the nervous system has not been well understood so far. Expression of Dlk1 was detected in the diencephalon, the metencephalon, the medial tegmental neuroepithelium and the spinal cord of the developing rat embryo according to Jensen and colleagues (Jensen et al. 2001). In the adult rat and human brain it could be detected in the ventral mesencephalon and the pons via in-situ hybridization and by immunohistochemistry in the ventral tegmental area, the substancia nigra pars compacta and the Edinger-Westphal nuclei. A functional study by Christophersen et al., revealed Dlk1 to be upregulated upon lentivirus-mediated overexpression of glia cell derived neurotrophic factor (GNDF) in mouse striatum (Christophersen et al. 2007). Furthermore in the same publication Dlk1 could be colocalized with thyroxin hydroxylase positive cells in the murine mesencephalon during embryonic development. The analysis of the human mesencephalic cell line MesC2.10 in the same article showed that expression of Dlk1 precedes the expression of thyroxin hydroxylase under culture conditions that induce cell differentiation into dopamine neurons. Yet conditioning the culture medium with soluble Dlk1 was not sufficient to introduce differentiation. In a more recent study Dlk1 function was associated with an increased number in dopaminergic neurons in the developing mouse brain (Bauer et al. 2008). This suggests a role of Dlk1 in the process of differentiation of dopamine neurons in the brain and points to a role in neuronal fate specification in other regions of the CNS. 


\section{Introduction}

\subsection{Aims of this work}

Despite their decisive importance for proper body movement and posture, the molecular mechanisms underlying the specification of functional motor neuron types (FMNTs) have remained unknown. Advances in that field have so far been minor as it has been difficult to identify and characterize the different FMNTs due to the lack of molecular markers.

Therefore, the initial aim of this work was the identification of such marker genes specific for distinct FMNTs. Thus, the gene expression profile of different FMNTs was compared by differential transcriptome analysis of motor neuron populations that were enriched in either fast or slow motor neurons

Subsequently, the marker genes found in this screen got to be verified and analyzed in terms of their expression pattern within the spinal cord generally and in motor neurons in particular. Thereby expression of these genes should be linked directly to a distinct FMNT.

Ultimately, the aim of this work was to evaluate the potential functional role of these marker genes in function and development of the FMNTs and motor neurons in general. These results should not only provide a more profound knowledge regarding FMNTs, but also allow deeper insights into the mechanisms that underlie the formation of neuronal diversity within the central nervous system. 


\section{Materials}

\subsection{Consumables and plastic ware}

Consumables and general plastic labware were bought from Starlab $\mathrm{GmBH}$ and Sarstedt AG. Instruments for mouse and chick surgery were purchased from Fine Science Tools GmBH.

\subsection{Antibodies}

\subsubsection{Primary antibodies}

$\begin{array}{llll}\text { Antigen } & \text { Host } & \text { Dilution } & \text { Supplier } \\ \text { Dlk1 } & \text { Rabbit } & 1: 750 & \text { Santa Cruz Biotechnology GmbH } \\ \text { ChAT } & \text { Goat } & 1: 300 & \text { Millipore AG } \\ \text { Digoxigenin } & \text { Sheep } & 1: 2500 & \text { Roche Applied Science GmbH } \\ \text { NeuN } & \text { Mouse } & 1: 1500 & \text { Millipore AG } \\ \text { Is11/2 } & \text { Mouse } & 1: 200 & \text { Developmental Studies Hybridoma Bank }\end{array}$

\subsubsection{Secondary Antibodies}

$\begin{array}{llll}\text { Target species } & \text { Host } & \text { Conjugate } & \text { Supplier } \\ \text { Mouse } & \text { Donkey } & \text { Alexa fluorescent dye } & \text { Invitrogen } \mathrm{GmbH} \\ \text { Goat } & \text { Donkey } & \text { Alexa fluorescent dye } & \text { Invitrogen } \mathrm{GmbH} \\ \text { Rabbit } & \text { Donkey } & \text { Alexa fluorescent dye } & \text { Invitrogen } \mathrm{GmbH}\end{array}$

The secondary antibodies were applied at a dilution of 1:2000 in antibody staining solution.

\subsection{Mouse lines}

\subsubsection{Hb9-GFP transgenic mice}

Hb9-GFP is a transgenic mouse line expressing the enhanced green fluorescent protein under control of the $\mathrm{Hb} 9$ promoter. Therefore the GFP transgene is expressed in postmitotic motor neurons (Lee et al. 2004). 


\section{Materials}

\subsubsection{Dlk1 knockout mice}

In order to create this systemic knockout mouse line the $D l k l$ gene has been replaced by a neomycin resistance antibiotic selection marker. The $D l k l^{-/-}$mice are viable and give birth to viable pups. The mouse line was initially described by Raghunandan et al. (2008). The knockout strategy is illustrated in Fig.3.1.

Knockout construct
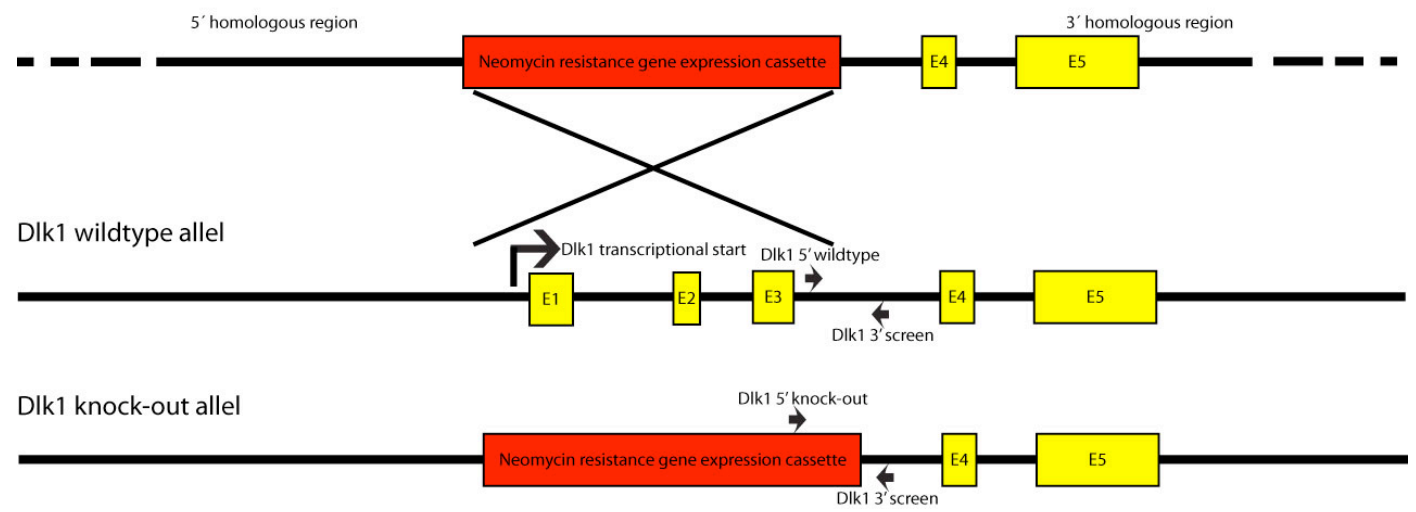

Fig. 3.1 Dlk1 knockout strategy The first three exons of the Dlkl allele have been replaced by a neomycin resistance cassette. A new primer-binding site for genotyping site is introduced along with the neomycin cassette. (Modified after Raghunandan et al. 2008)

\subsubsection{CD-1 Mice}

The initial expression screen was performed using mice of the CD-1 strain. These mice belong to a standard lab strain and do not have any genetic modifications.

\subsection{Chemicals \& Reagents}

Chemical

Agar-Agar

Agarose NEEO

$\beta$-Mercaptoethanol

Bovine serum albumin (BSA)

Cholera toxin B subunit Alexa conjugated

Diethylpyrocarbonate (DEPC)

DIG RNA labeling mix

DNA Ladder $1 \mathrm{~kb}$

dNTP Mix

Ethanol $100 \%$

\section{Supplier}

Carl Roth GmbH

Carl Roth GmbH

Carl Roth GmbH

Carl Roth GmbH

Invitrogen $\mathrm{GmbH}$

Carl Roth GmbH

Roche Diagnostics

Fermentas $\mathrm{GmbH}$

Fermentas GmbH

Carl Roth GmbH 


\section{Materials}

Ethylenediaminetetraacetate (EDTA)

Fermentas $\mathrm{GmbH}$

Formamide

Applichem GmbH

Glycerol

Carl Roth GmbH

Heparin

Applichem GmbH

Isopropanol

Carl Roth GmbH

Histoacryl tissue glue

B. Braun GmBH

Hydrogen peroxide

Applichem GmbH

L-15 medium

Applichem GmbH

Magnesiumchloride

Carl Roth GmbH

Maleic acid

Carl Roth $\mathrm{GmbH}$

Methanol

Carl Roth GmbH

NBT/BCIP tablets

Roche Diagnostics GmbH

OCT embedding medium

Sakura Finetek GmbH

Orange $\mathrm{G}$

Applichem GmbH

PBS pH 7.2

Invitrogen $\mathrm{GmbH}$

Proteinase $\mathrm{K}$

Applichem GmbH

Salmon sperm DNA

Applichem GmbH

Sodiumchloride

Carl Roth GmbH

Sodium dodecyl sulfate

Carl Roth GmbH

Sodium citrate

Carl Roth GmbH

Sucrose

Carl Roth GmbH

Tris

Carl Roth GmbH

Triton X-100

Carl Roth GmbH

Tryptone

Applichem GmbH

Tween 20

Carl Roth GmbH

VectaShield

Vector Labs Inc.

Yeast extract

Applichem $\mathrm{GmbH}$ 


\subsection{Enzymes}

Enzyme

Supplier

Green Taq DNA Polymerase

Fermentas $\mathrm{GmbH}$

Restriction Enzymes

Fermentas $\mathrm{GmbH}$

SP6 RNA Polymerase

Roche Diagnostics GmbH

Shrimp alkaline phosphatase

Fermentas $\mathrm{GmbH}$

T4 DNA ligase

Fermentas $\mathrm{GmbH}$

T7 RNA Polymerase

Roche Diagnostics GmbH

Phusion High Fidelity DNA Polymerase

New England Biolabs GmbH

\subsection{Solutions}

\section{Solution}

Antibody staining solution

Blocking buffer

DNA running buffer

In-situ hybridization buffer

In-situ wash buffer I

In-situ wash buffer II

LB agar

LB medium

PBT

SSC 20x stock solution

MBST

NTMT

TAE buffer

Tail biopsy lysis buffer

\section{Contents}

PBS pH 7.2; $1 \%$ BSA; $1 \%$ Triton X-100

Roche blocking reagent $1 \%(\mathrm{w} / \mathrm{v})$ in PBT

$8 \%$ Sucrose in TAE; $1 \mathrm{mg} / \mathrm{ml}$ Orange $\mathrm{G}$

$50 \%$ Formamide; SSC 5x pH 4.5; $1 \%$ SDS;

$10 \mathrm{mg} / \mathrm{ml}$ Heparin; $10 \mathrm{mg} / \mathrm{ml}$ salmon sperm

DNA

$50 \%$ Formamide; SSC 5x pH 4.5; $1 \%$ SDS

$50 \%$ Formamide; SSC 2x pH 4.5; $0.1 \%$

Tween-20

LB medium with $1 \%(w / v)$ agar-agar

$5 \mathrm{~g} / 1 \mathrm{NaCl} ; 5 \mathrm{~g} / 1$ yeast extract; $10 \mathrm{~g} / 1$ tryptone

PBS pH 7.2; $0.1 \%$ Tween-20

$3 \mathrm{M} \mathrm{NaCl} ; 300 \mathrm{mM}$ Sodium citrate; $\mathrm{pH} 4.5$

$0.1 \mathrm{M}$ Maleic acid; $0.15 \mathrm{M} \mathrm{NaCl} \mathrm{pH} 7.5$

$100 \mathrm{mM}$ Sodium chloride; $10 \mathrm{mM}$ Tris- $\mathrm{HCl}$;

$50 \mathrm{mM} \mathrm{MgCl} 2 ; \mathrm{pH} 9.5$

$40 \mathrm{mM}$ Tris; 1mM EDTA adjust $\mathrm{pH} 7.5$ with glacial acetic acid

$0.1 \mathrm{M}$ Tris $\mathrm{pH} 8 ; 30 \mathrm{mM} \mathrm{NaCl}$; $0.2 \mathrm{mM}$ EDTA;

$0.05 \% \mathrm{SDS} ; 0.5 \mathrm{mg} / \mathrm{ml}$ proteinase $\mathrm{K}$ 


\subsection{Kits}

Kit

iScript cDNA synthesis Kit

MouseRef-8 v2.0 Expression BeadChip Kit

PrimeScript $1^{\text {st }}$ strand cDNA synthesis Kit

Qiaprep Spin Miniprep Kit

QiaQuick Gel extraction Kit

RNeasy Micro Kit

TotalPrep RNA Amplification Kit

\section{Supplier}

Bio-Rad GmbH

Illumina, San Diego

Takara Bio Inc.

Qiagen $\mathrm{GmbH}$

Qiagen $\mathrm{GmbH}$

Qiagen $\mathrm{GmbH}$

Illumina Inc.

\subsection{Software}

Program

Bead studio

BioEdit

Image J

pDraw

Photoshop

Prism
Application

Microarray data analysis

DNA Sequence analysis

Image processing

Vector construction

Image processing

Data analysis
Supplier

Illumina, San Diego

Hall (1999)

Abramoff et al. (2004)

Acaclone Inc.

Adobe Inc.

Graph Pad

\subsection{Vectors}

\section{Plasmid}

GATA2-GFP

Hb9-GFP

pCAGEN

pGK-Cre

pCRII-TOPO

\section{Supplier or Reference}

Zhou et al. (2000)

Lee et al. (2004)

Addgene Inc.

Addgene Inc.

Invitrogen $\mathrm{GmbH}$ 


\section{Methods}

\subsection{Mouse animal experiments}

All animal experiments have been carries out in accordance with the animal protection law of Germany and were approved by the district government.

The experimental animals were housed under standard conditions with access to food and water ad libitum.

\subsubsection{Mouse genotyping}

\subsubsection{DNA extraction from mouse tissue}

DNA was extracted from tail biopsies by incubation of tail tips in tail biopsy lysis buffer over night at $56^{\circ} \mathrm{C}$. The lysate was cleared by centrifugation for $10 \mathrm{~min}$ at $16.000 \mathrm{x} g$ and the supernatant was mixed with $500 \mu 1$ isopropanol and centrifuged for $2 \mathrm{~min}$ at $16.000 \mathrm{x}$ g. Subsequently the DNA pellet is washed with $500 \mu 170 \%$ Ethanol. Afterwards the DNA is air-dried and $50 \mu \mathrm{l}$ of water were added.

\subsubsection{Genotyping of Hb9-GFP animals}

The genotyping was performed via PCR with the reaction components given below:

$3 \mu 1 \quad$ Green Taq reaction buffer

$2 \mu \mathrm{l} \quad 25 \mathrm{mM} \mathrm{MgCl}_{2}$

$1 \mu 1 \quad$ Mouse tail DNA

$1 \mu 1 \quad 10$ mM EGFP forward primer (CCT ACG GCG TGC AGT GCT TCA GC)

$1 \mu 1 \quad 10 \mathrm{mM}$ EGFP reverse primer (CGG CGA GCT GCA CGC TGC GTC CTC)

$0.5 \mu 1 \quad 10 \mathrm{mM}$ dNTP mix

$0.5 \mu 1$ Green Taq DNA polymerase (Fermentas GmbH)

adjust to $30 \mu$ total volume with $\mathrm{ddH}_{2} \mathrm{O}$

The genotyping reaction is performed with the following parameters:

Intial denaturation

$94^{\circ} \mathrm{C} \quad 3 \mathrm{~min}$

28 cycles

$94^{\circ} \mathrm{C} \quad 1 \mathrm{~min}$ 
$62^{\circ} \mathrm{C} \quad 1 \mathrm{~min}$

$72^{\circ} \mathrm{C} \quad 30 \mathrm{sec}$

Final elongation

$72^{\circ} \mathrm{C} \quad 5 \mathrm{~min}$

Hb9-GFP positive animals exhibited a DNA band after running it on an agarose gel, while negative reactions were not showing a visible signal. The PCR was carried out in a Mastercycler gradient thermocycler (Eppendorf AG)

\subsubsection{Genotyping of Dlk1 knockout animals}

Polymerase chain reaction was applied to genotype the Dlk1 knockout animals. The reaction was set up as stated below:

$3 \mu 1 \quad$ Green Taq reaction buffer

$2 \mu \mathrm{l} \quad 25 \mathrm{mM} \mathrm{MgCl}_{2}$

$1 \mu 1 \quad$ Mouse tail DNA

$1 \mu 1 \quad 10 \mathrm{mM}$ dlk1 5' knockout primer (CAT CTG CAC GAG ACT AGT G)

$1 \mu \mathrm{l} 10 \mathrm{mM}$ dlk1 5' wild-type primer (CCA AAT TGT CTA TAG TCT CCC TC)

$1 \mu \mathrm{l} 10 \mathrm{mM}$ dlk1 3' screen primer (CTG TAT GAA GAG GAC CAA GG)

$0.5 \mu 1 \quad 10 \mathrm{mM}$ dNTP mix

$0.5 \mu \mathrm{l}$ Green Taq DNA polymerase (Fermentas $\mathrm{GmbH}$ )

adjust to $30 \mu$ total volume with $\mathrm{ddH}_{2} \mathrm{O}$

The genotyping polymerase chain reaction is performed with the following parameters:

Initial denaturation

$94^{\circ} \mathrm{C} \quad 2 \mathrm{~min}$

18 cycles

$94^{\circ} \mathrm{C} \quad 30 \mathrm{sec}$

$64^{\circ} \mathrm{C}-55.5^{\circ} \mathrm{C} \quad 30 \mathrm{sec}$ temperature drop of $0.5^{\circ} \mathrm{C}$ each cycle

$72^{\circ} \mathrm{C} \quad 30 \mathrm{sec}$

25 cycles

$94^{\circ} \mathrm{C} \quad 30 \mathrm{sec}$

$55^{\circ} \mathrm{C} \quad 30 \mathrm{sec}$ 
Final elongation

$72^{\circ} \mathrm{C}$

$10 \mathrm{~min}$

The reaction yielded a $495 \mathrm{bp}$ agarose gel band in case of knockout animals and a $414 \mathrm{bp}$ band in wild type animals. Genotyping of heterozygous animals resulted in both bands.

\subsubsection{Retrograde labeling experiments}

\subsubsection{Retrograde tracing of motor neuron with cholera toxin B}

Lyophilized Alexa conjugated cholera toxin B subunit is resuspended in PBS pH 7.2 to a final concentration of $1 \mu \mathrm{g} / \mu \mathrm{l}$, aliquoted and stored at $-20^{\circ} \mathrm{C}$. Prior to the muscular injection the tracing agent was filled into a glass injection capillary (World Precision Instruments Inc.). The skin of the anesthetized animal was opened and the target muscle exposed. Then about $1 \mu 1$ of the cholera toxin was injected. Excess liquid outside the muscle was removed and the wound was sealed using tissue glue (Histoacryl, B. Braun GmbH). The animals were kept in an incubator set to $35^{\circ} \mathrm{C}$ for $20 \mathrm{~min}$ and then given back to the mother. Retrograde tracing was performed for at least one day for P4 animals until they were sacrificed.

\subsubsection{Isolation of retrogradely traced motor pools}

Mice were sacrificed by decapitation and pinned ventral side up onto silicone dish using insect pins. Then the animal was eviscerated and the vertebra opened from the ventral side. The lumbar part of the spinal cord was removed and pinned in a fresh silicone dish filled with ice-cold DEPC-PBS. The silicone dish was transferred beneath a fluorescence stereomicroscope and the labeled motor neuron pools were excised with nuclease free microscissors and blades.

\subsubsection{Mouse behavioral experiments}

\subsubsection{Grip strength assay}

4-month-old Dlkl knockout and age matched wild type control mice were suspended at the tail and placed in close proximity of the handling bar of a Columbus Instruments grip strength meter. The mice were kept in this position until they grasped the handle bar and were carefully pulled backwards horizontally until they let go the 


\section{Methods}

bar and the peak force before release was measured. The test was repeated at three subsequent days with five trials for each mouse. Only the highest value was taken.

\subsubsection{Running endurance test}

Four-month-old male Dlkl knockout mice and age matched Dlkl wild type control animals were placed onto a treadmill with adjustable speed. An electric current of $3 \mathrm{~V}$ was applied to grid at the end of the treadmill to keep the animals running. In order to get the animals used to the environment the mice were trained three subsequent days on the treadmill for $5 \mathrm{~min}$ without an incline. On the trial day the mice were subjected to a running paradigm of $5 \mathrm{~min}$ at a speed of $10 \mathrm{~m} / \mathrm{min}$ follow by a gradual increase of speed by $2 \mathrm{~m} / \mathrm{min}$ every $2 \mathrm{~min}$ up to a maximum speed of $34 \mathrm{~m} / \mathrm{min}$. For the medium intensity running endurance test the treadmill was adjusted to an incline of $10^{\circ}$ while for the high intensity the incline was set to $20^{\circ}$. The mice were claimed as exhausted when they remained on the grid despite of the electrical current, an air puff and mechanical pruning (five times). The time on the treadmill was taken and the distance traveled was calculated. The test was only carried out once in order to exclude training effects.

\subsubsection{Clasping assay}

In order to test the clasping phenotype six month old male $D l k 1^{-/-}$and $D l k 1^{+/+}$mice were taken out of their cage and suspended on the tail for $30 \mathrm{sec}$ about $50 \mathrm{~cm}$ above the ground. If a clasping event was monitored within the first $15 \mathrm{sec}$ on either the fore or the hindlimbs it was counted as two points, if there was a clasping event between $15 \mathrm{sec}$ and $30 \mathrm{sec}$ it was counted as one point. Subsequently fore and hindlimb scores where added in order to get an overall score.

So the maximal score possible was four points for both fore and hindlimb clasping within the first 15 seconds of suspension. Directly after being tested for their unloaded clasping phenotype the mice were put onto a treadmill for $5 \mathrm{~min}$ with a speed of $14 \mathrm{~m} / \mathrm{min}$ at an incline of $20^{\circ}$. The electrical grid normally used to force the mice to stay on the treadmill at a constant speed was deactivated in order to prevent possible clasping behavior caused by electrical stimulation. Instead of this air puffs and mechanical pruning were used. Directly after the exercise the animals were tail 
suspended and the clasping phenotype was scored as before. The test was performed once per day on three subsequent days and the average score was calculated.

\subsection{Quantitative expression analysis}

\subsubsection{RNA purification from microdissected motor pools}

The Qiagen RNeasy Micro Kit was used to extract and purify the total RNA from isolated motor pools. After dissection the motor pools were transferred into lysis buffer (RLT) with $\beta$-Mercaptoethanol already added. In order to solubilize the tissue the reaction tube was vigorously vortexed and put on $-80^{\circ} \mathrm{C}$ for $30 \mathrm{~min}$. Subsequently the RNA was extracted according to the manufacturer's protocol for total RNA isolation from animal tissues. Carrier RNA was not used as it interferes with downstream reactions. Finally the RNA concentration was determined and the RNA was stored at $-80^{\circ} \mathrm{C}$ until further use.

\subsection{2 cDNA synthesis for real-time experiments}

$100 \mathrm{ng}$ of total RNA were used for reverse transcription with the Bio-Rad iScript cDNA Synthesis Kit.

The components were added in the following order:

$4 \mu 1 \quad 5 x$ iScript Reaction Mix

$1 \mu \mathrm{l} \quad$ iScript Reverse Transcriptase

100 ng Template RNA

The reaction volume was adjusted to $20 \mu 1$ with nuclease free water. The reaction was set up on ice and the transferred to a thermocycler were the cDNA was synthesized according to the following protocol:

$25^{\circ} \mathrm{C} \quad 5 \mathrm{~min}$

$42^{\circ} \mathrm{C} \quad 30 \mathrm{~min}$

$85^{\circ} \mathrm{C} \quad 5 \mathrm{~min}$

Finally the samples were chilled on ice and the total volume was adjusted to $100 \mu 1$ by addition of nuclease free water. The cDNA was stored at $-20^{\circ} \mathrm{C}$. 
4. Methods

\subsubsection{Quantitative real-time PCR}

Quantitive real-time PCR experiments were performed on a Roche LightCycler 480 using the universal probe library system. The gene expression was normalized to the expression of the alpha Tubulin 1B.

The following oligonucleotides were used with the according Roche universal probe library (UPL) probes:

Delta like homolog 1

Dlk1 qPCR left CGG GAA ATT CTG CGA AAT AG

Dlk1 qPCR right TGT GCA GGA GCA TTC GTA CT

UPL Probe $\quad 80$

Neurotensin

Nts qPCR left TGA CTC TCC TGG CTT TCA GC

Nts qPCR right $\quad$ TCC AGG GCT CTC ACA TCT TC

UPL Probe 99

Alpha Tubulin $1 B$

Tubalb qPCR left AGG AGC TGG CAA GCA TGT

Tubalb qPCR right AGC TGC TCA GGA TGG AAG AG

UPL Probe 85

Reactions were set up and run according to the Roche LightCycler protocol.

\subsubsection{Synthesis of biotin-labeled aRNA for Microarray hybridization}

Biotin-labeled aRNA was generated with the Illumina TotalPrep RNA Amplification Kit. Therefore an amount of $100 \mathrm{ng}$ total RNA of each sample was applied. As one chip is consisting of eight individual arrays, all eight samples to be hybridized on one chip were processed in parallel in order to minimize technical variations. The RNA was processed according to the manufacturer's protocol. Finally the biotinylated aRNA was eluted with nuclease free water and the concentration was determined. The final aRNA concentration was adjusted to $200 \mathrm{ng} / \mu \mathrm{l}$. 


\section{Methods}

\subsubsection{Microarray hybridization}

$1 \mu \mathrm{g}$ of total Biotin-labeled aRNA generated as described in 4.2.4 was used from each sample. The samples were applied to MouseRef-8 v2.0 expression bead chips and processed according to the manufacturer's protocol. Microarray hybridization and imaging were performed by Dr. Lars Wittler and Dr. Phillip Grote at the Max-Planck Insitute for Molecular Genetics, Berlin.

\subsection{Molecular biology}

The methods described in this section were performed according to Sambrook et al. (1989) if not otherwise stated.

\subsubsection{Preparation of chemically competent $E$.coli cells}

Competent E. coli cells of the Top10 strain (Invitrogen $\mathrm{GmbH}$ ) were prepared according to the protocol by Inoue et al. (Inoue et al. 1990) and checked for competence by a test transformation with the pBluescript KS (-) plasmid. The cells were stored at $-80^{\circ} \mathrm{C}$ until use.

\subsubsection{Transformation of chemically competent $E$.coli}

Transformation of E.coli was performed by adding $100 \mu \mathrm{l}$ of competent cells to a maximum amount of $10 \mu \mathrm{l}$ of DNA solution. The cells were incubated on ice for $30 \mathrm{~min}$ and heat shocked at $43^{\circ} \mathrm{C}$ for $1 \mathrm{~min}$. Afterwards they were chilled on ice for $1 \mathrm{~min}$ and $1 \mathrm{ml} \mathrm{LB}$ medium was added. Subsequently they were incubated for $1 \mathrm{~h}$ on a $37^{\circ} \mathrm{C}$ shaker and plated on an agar plate containing the appropriate selection antibiotic. E.coli cells were grown over night at $37^{\circ} \mathrm{C}$ in an incubator.

\subsubsection{Cryopreservation and recovery of E.coli clones}

$0.5 \mathrm{ml}$ of an E.coli overnight culture were mixed with $0.5 \mathrm{ml}$ of $100 \%$ glycerol in a reaction tube and submersed in liquid nitrogen. Subsequently the sample was stored at $-80^{\circ} \mathrm{C}$ until reuse. In order to regrow the culture approximately $50 \mu \mathrm{l}$ of the frozen stock were grown for $12 \mathrm{~h}$ in $5 \mathrm{ml} \mathrm{LB}$ medium for miniprep DNA purification or for $16 \mathrm{~h}$ in $200 \mathrm{ml} \mathrm{LB}$ medium for maxiprep purification. 


\section{Methods}

\subsubsection{Plasmid isolation}

Bacterial cells were grown from single colonies or glycerol stocks. Small-scale plasmid isolation was performed using $5 \mathrm{ml}$ of an E. coli overnight culture using the Qiagen Spin Miniprep kit according to the manufacturer's instructions. Finally the DNA was eluted in $50 \mu 1$ of buffer.

For large-scale plasmid preparations $250 \mathrm{ml}$ of $E$. coli liquid culture were grown over night. Plasmid DNA was extracted with the Qiagen Plasmid Maxi kit according to the manufacturer's protocol.

\subsubsection{DNA restriction digest}

For analytical DNA digestions $2 \mu 1$ (app. $500 \mathrm{ng}$ ) of DNA were incubated with 2 units (one unit cleaves $1 \mu \mathrm{g}$ of DNA per hour) of the appropriate restriction enzyme. The incubation was carried out in the reaction buffer and at the temperature recommended by the manufacturer for $1 \mathrm{~h}$.

For preparative digestions $10 \mu \mathrm{g}$ of DNA were incubated with 10 units of the restriction enzyme for at least three hours at the appropriate reaction temperature. If a double digestion was not possible according to the manufacturer's recommendations, the DNA was ethanol precipitated and resuspended in nuclease free water prior to the second restriction digest.

\subsubsection{Alkaline phosphatase treatment}

To prevent religation of vector DNA leading to false positive colonies, the DNA was treated with shrimp alkaline phosphatase after restriction digestion. Therefore 0.5 units of the phosphatase enzyme were added to the restriction digest per $10 \mu \mathrm{g}$ of DNA. Then the reaction mixture was incubated for $15 \mathrm{~min}$ at $37^{\circ} \mathrm{C}$.

\subsubsection{Agarose gel electrophoresis}

$0.5-2 \%(\mathrm{w} / \mathrm{v})$ of agarose were dissolved in $1 \mathrm{x}$ TAE buffer by heating in a microwave oven. After cooling the agarose solution for $5 \mathrm{~min} 2 \mu \mathrm{l}$ of $1 \%(\mathrm{w} / \mathrm{v})$ ethidiumbromide solution were added per $100 \mathrm{ml}$ of agarose. The solution was poured into a gel tray and a comb was inserted. After solidification the comb was removed and the gel was put into a chamber filled with running buffer. DNA running buffer was added to the samples, which were subsequently loaded onto the gel together with the DNA ladder. 
Then an electrical current was applied. Segregation of DNA bands was checked under UV light. Gel pictures were taken with a Gel Jet Imager (Intas GmbH).

\subsubsection{Purification of DNA from agarose gels}

DNA fragments were extracted from agarose gels after the size could be clearly determined and they have reached a sufficient segregation. The DNA bands were cut out from the gel and the DNA was purified with the QIAquick gel extraction kit (Qiagen) according to the manufacturer's protocol.

\subsubsection{Determination of nucleic acid concentration}

Concentration of nucleic acids was determined based on UV absorption using a Nanodrop ND-1000 UV-Vis spectrophotometer (Peqlab Biotechnologie GmbH).

\subsubsection{DNA ligation}

Vector and insert DNA concentration were determined as described in section 4.3.9 Reaction mixtures were prepared with molar vector to insert concentrations of 1:3, 1:5 and 1:10. Subsequently T4 DNA ligase and reaction buffer were added. The reaction was incubated at room temperature for sticky end ligations or at $16^{\circ} \mathrm{C}$ for blunt end ligations for $2 \mathrm{~h}$.

\subsection{Immunohistochemistry}

\subsubsection{Cryosectioning of mouse spinal cords and embryos}

Mice were sacrificed by decapitation or cervical dislocation. Subsequently the animals were eviscerated and the spinal cords were removed and collected in ice-cold PBS. Afterwards the cords were fixed by immersion in $4 \%$ paraformaldehyde solution. The fixation time was $6 \mathrm{~h}$ for spinal cords from mice up to P10 and over night for spinal cords from older animals. Then the spinal cords were washed for $24 \mathrm{~h}$ in PBS followed by dehydration in PBS containing $30 \%$ Sucrose for $6 \mathrm{~h}$. All incubation steps were carried out at $4^{\circ} \mathrm{C}$ on a vertical shaker. After dehydration the spinal cords were equilibrated for $5 \mathrm{~min}$ in OCT and placed into an embedding mold filled with OCT. The embedding molds were placed on dry ice in order to solidify the OCT. Embedding molds were stored at $-20^{\circ} \mathrm{C}$ or $-80^{\circ} \mathrm{C}$ until cutting. 


\section{Methods}

For cutting the solidified OCT blocks were removed from the molds and mounted in a CM $1510 \mathrm{~S}$ cryostat (Leica Microsysteme $\mathrm{GmbH}$ ). The tissue was cut into $30 \mu \mathrm{m}$ slices for postnatal mouse spinal cords and chicken embryos and $20 \mu \mathrm{m}$ slices for mouse embryos. The slices were attached to SuperFrost Plus cover slides and air dried for $1 \mathrm{~h}$ at room temperature. Subsequently they were stored at $-20^{\circ} \mathrm{C}$ until use.

\subsubsection{Cryosectioning of chick embryo spinal cords}

The eggs were chilled at room temperature for $1 \mathrm{~h}$ to reduce movement of the embryos. Subsequently the embryos were removed and decapitated. The vertebra was extracted and washed in cold PBS. Then the vertebra was fixed in $4 \%$ paraformaldehyde for $6 \mathrm{~h}$ (E12) or $8 \mathrm{~h}$ (E18). The subsequent steps were the same as described in 4.4.1 for mouse spinal cords.

\subsubsection{Immunohistological staining procedure}

Cryosections were washed two times for $10 \mathrm{~min}$ in PBS in order to rehydrate and to remove residual embedding material. Afterwards the primary antibody solution is applied. Primary antibodies were diluted in PBS pH 7.2 containing $1 \%$ BSA and $1 \%$ Triton X-100 to the concentration given in Materials (3.2.1). The sections were incubated at $4^{\circ} \mathrm{C}$ over night.

The next day the primary antibody solution was removed and the sections were washed trice with PBS for 10min each. Afterwards the appropriate secondary antibodies were applied in the concentrations given in Materials (3.2.2) in antibody staining solution. The sections were incubated for $1 \mathrm{~h}$ at room temperature. Afterwards they were washed trice with PBS and mounted with VectaShield or $50 \%$ glycerol in PBS.

\subsection{In-situ hybridization}

\subsubsection{In-situ probe generation}

The Dlk1 in-situ probe was constructed according to the Allen Brain Atlas Riboprobe (ID RP_080122_04_F07) by PCR amplification from P4 mouse spinal cord cDNA (Lein et al. 2007). The following oligonucleotides were used:

Dlk1 ISH forward GCA TCT GCA AGG ATG GCT

Dlk1 ISH reverse TGT AGC GCA GGT TGG ACA 
The probes were T/A cloned into the pCRII-TOPO vector. After verification of the cloned DNA and its orientation the vector was linearized with using an appropriate restriction enzyme and the antisense RNA probe was created using DIG-RNA labeling mix and the SP6 RNA polymerase according to the manufacturer's protocol. After synthesis the reaction volume was brought to $100 \mu 1$, the concentration was determined and the probes integrity and length was verified by agarose gel electrophoresis.

\subsubsection{Sample preparation for in-situ analysis of postnatal mouse tissue}

Mouse spinal cords were extracted and washed in DEPC-PBS. Then the spinal cords were put into $4 \%$ paraformaldehyde for $48 \mathrm{~h}$. Subsequently roots and membranes were removed and the spinal cords are embedded in $3 \%$ agarose. The spinal cords were sectioned into $60 \mu \mathrm{m}$ slices using a VT1200S vibratome (Leica Microsysteme). The slides were dehydrated by incubation in rising concentrations of methanol $(25 \%$, $50 \%, 75 \%, 100 \%$ ) for $10 \mathrm{~min}$ each and stored at $-20^{\circ} \mathrm{C}$.

\subsubsection{Sample preparation for in-situ analysis of prenatal mouse tissue}

Embryos were washed in DEPC-PBS and subsequently fixed for $48 \mathrm{~h}$ in $4 \%$ PFA in PBS. Then the embryos were dehydrated in PBS containing $30 \%$ sucrose and $4 \%$ PFA for $24 \mathrm{~h}$. All steps were carried out at $4^{\circ} \mathrm{C}$ on a shaker. The embryos were mounted in OCT and cut into $10 \mu \mathrm{m}$ sections. The slides with the sections were air dried for $30 \mathrm{~min}$ and stored at $-80^{\circ} \mathrm{C}$.

\subsubsection{In-situ probe hybridization and detection for postnatal mouse tissue}

The spinal cord slices were rehydrated by incubation in a descending row of methanol in PBT from $100 \%$ methanol to $25 \%$ methanol for 10 min each step. Subsequently the slices were washed with DEPC-PBT three times for 5 min each. Then the samples were bleached with $6 \% \mathrm{H}_{2} \mathrm{O}_{2}$ for $10 \mathrm{~min}$ and washed again three times with PBT before they were treated for 5 min with $0.5 \%$ Triton-X100 in PBT. After two more washing steps in PBT $20 \mu \mathrm{g} / \mathrm{ml}$ proteinase $\mathrm{K}$ in PBT was added to the samples and 


\section{Methods}

they were incubated for $5 \mathrm{~min}$ before the reaction was stopped by removing the enzyme and addition of $2 \mathrm{mg} / \mathrm{ml}$ glycine in PBT. After three more washes with PBT the slices were postfixed for 30 min with $4 \%$ PFA. Then they were washed twice for 5 min and put into hybridization buffer that has been prewarmed to $65^{\circ} \mathrm{C}$ for $2 \mathrm{~h}$. During prehybridization the cRNA probe was diluted to a final concentration of $1 \mathrm{ng} / \mu 1$. The prehybridization solution was removed and the probe was applied. The slides were incubated o.n. at $65^{\circ} \mathrm{C}$. The next day probe was removed and the slides were washed three times for $30 \mathrm{~min}$ each with in-situ wash buffer 1 at $65^{\circ} \mathrm{C}$. Then they were washed three times for $30 \mathrm{~min}$ each with in-situ wash buffer 2 at $65^{\circ} \mathrm{C}$. Then the slices were washed three times for $5 \mathrm{~min}$ each with PBT. The slices were preincubated for $2 \mathrm{~h}$ with blocking buffer at RT. Then the anti-DIG antibody was added in blocking buffer at a final dilution of 1:5000 and the samples were incubated over night at $4^{\circ} \mathrm{C}$. The next day the samples were washed five times with PBT and two times with NTMT for $5 \mathrm{~min}$ each. Now NBT/BCIP solution was added and the slides were incubated at RT until the staining was visible. Then the slices were washed five times with PBS and mounted on glass slides.

\subsubsection{In-situ probe hybridization and detection for prenatal mouse tissue}

The slides were removed from the freezer and washed twice for $5 \mathrm{~min}$ each with DEPC-PBS before they were fixed with $4 \%$ PFA for $5 \mathrm{~min}$. Subsequently the samples were washed three times for $5 \mathrm{~min}$ in DEPC-PBS and incubated with proteinase $\mathrm{K}(10 \mu \mathrm{g} / \mathrm{ml})$ for $10 \mathrm{~min}$. Afterwards the slides were washed for three times with PBS and postfixed for $5 \mathrm{~min}$ with $4 \%$ PFA. Thereafter the samples were washed again three times with PBS. Then another washing step with $0.85 \% \mathrm{NaCl}$ followed. The next washing step was performed with $70 \%$ Ethanol and $0.85 \% \mathrm{NaCl}$ in water for $5 \mathrm{~min}$ before the final washing step was performed with $95 \%$ ethanol for 5 min. Subsequently the samples were air dried and then cover with the probe solution that was dissolved in hybridization buffer to a final concentration of $0.5 \mathrm{ng} / \mu \mathrm{l}$ and covered with parafilm. The slides were incubated in a humidified chamber at $65^{\circ} \mathrm{C}$ over night. The next day the parafilm was removed by washing the slide for $5 \mathrm{~min}$ at $65^{\circ} \mathrm{C}$ in $5 \mathrm{x}$ SSC. Afterwards the slides were washed for $30 \mathrm{~min}$ at $65^{\circ} \mathrm{C}$ in $1 \mathrm{x} \mathrm{SSC}$ with $50 \%$ formamide. The subsequent washing step was performed in $2 x \mathrm{SSC}$ at 
$65^{\circ} \mathrm{C}$ for $20 \mathrm{~min}$ followed by another step for the same time at the same temperature in $0.2 \mathrm{xSC}$. Then the slides were washed three times in MBST for 5min each. Afterwards the slides were covered with blocking buffer and parafilm and incubated for $1 \mathrm{~h}$ at RT. Subsequently the blocking buffer was removed and anti-DIG antibody was applied diluted 1:5000 in blocking buffer. The samples were covered again with parafilm and incubated o.n. at $4^{\circ} \mathrm{C}$ in a humidified chamber. The next day the slides were washed in MBST three times for $5 \mathrm{~min}$ each and then washed in NTMT for 10 min. Then they were covered with NBT/BCIP solution and incubated until the staining developed. Afterwards they were washed five times with PBS and mounted.

\subsection{Imaging and image processing}

\subsubsection{Confocal imaging}

Confocal pictures were taken using a DMRIE2 microscope (Leica Mikrosysteme $\mathrm{GmbH}$ ) with a SP2 TCS scanner (Leica Mikrosysteme $\mathrm{GmbH}$ ) and appropriate objectives. The pictures were taken at an original resolution of $1024 \times 1024$ pixels.

\subsubsection{Brightfield and fluorescence imaging}

Images from in-situ hybridizations and in-situ hybridizations in combination with immunofluorescence were taken with an IXS1 microscope (Olympus Europa Holding $\mathrm{GmbH})$.

\subsubsection{Motor neuron size determination}

To quantify the motor neuron size, different muscles were injected with Cholera toxin B to retrogradely label their motor pools. The animals were sacrificed at stages P4 and P10. The spinal cords have been dissected and prepared for cryosections. $30 \mu \mathrm{m}$ cryosections were performed and the slides were air-dried. Then they were stained with the anti-Dlk1, anti-NeuN and anti-GFP antibodies over night. The next day the secondary antibody incubation was performed and the slides were mounted with VectaShield.

Pictures for motor neuron size determination and correlation of Dlk1 expression were taken using a confocal microscope. The optimal imaging parameters were determined and kept for all pictures taken. 


\section{Methods}

Only motor neurons with clear morphology, visible boundaries and a complete nucleus were counted in order to prevent artifacts. The cell soma area was quantified based on the labeled area by Alexa conjugated Cholera toxin B for retrogradely traced motor neurons and based on the GFP signal in the Hb9-GFP mouse line. The cell borders were manually determined. All pictures have been taken using a 200x magnification combined from ocular and objective magnification.

\subsection{Chick embryo electroporation}

Fertilized eggs of the Lohmann LSL strain were incubated for $65 \mathrm{~h}$ at $37.8^{\circ} \mathrm{C}$ Then $5 \mathrm{ml}$ of egg white were removed and a hole was cut above the embryo. The DNA solution was injected into the spinal channel and the embryo was covered with L-15 medium. Then the DNA was electroporated unipolar into the chick spinal cord using an ECM 830 electroporator (Havard Apparatus) with appropriate electrodes. The electroporation properties were voltage: $25 \mathrm{~V}$, pulse length: $50 \mathrm{~ms}$, no. of pulses: 5 , interval: $200 \mathrm{~ms}$ and polarity unipolar. Subsequently the eggs were sealed and put back into the incubator until the desired stage was reached.

\subsection{The long-term expression vector system}

In order to ensure high-level transgene expression within embryonic chick spinal cord postembryonic day 9 a novel vector system was created. This system was based on the tol 2 transposase, which stably integrates DNA fragments into the chicken genome (Kawakami \& Shima 1999, Sato et al. 2007, Takahashi et al. 2008). The tol2 transposase system requires specific recognition sites flanking the DNA fragment to be integrated into the genome. In this case the tol2 sites are flanking the entire expression cassette. The transposase is introduced into the cells by coelectroporation of the transposase gene bearing plasmid pCAGGS-T2TP driving transposase expression in all cell types.

To maintain expression specificity within motor neurons together with a high expression level the strong unspecific CAGGS promoter derived from the pCAGEN vector has been combined with a lox-STOP-lox cassette consisting of two loxP recognition sites for the $\mathrm{CRE}$ recombinase flanking three beta-globin polyadenylation signals (Hoess et al. 1982, Matsuda \& Cepko 2003). The Cre-loxP system has proven 


\section{Methods}

to work efficiently in chick primary motor neurons (Sato et al. 1998). Upon coelectroporation with a cell type specific Cre expressing plasmid the transgene was only expressed in an appropriate subset of cells. For this work three Cre driver constructs have been used, the non specific pGK-Cre, Hb9-Cre that expresses Cre in motor neurons and GATA2-Cre driving Cre expression in V2 interneurons (Soriano et al. 1991, Gu et al. 1993, Zhou et al. 2000). The Hb9-Cre and the GATA2-Cre vector were created based on eGFP expressing precursor plasmids by exchange of the GFP open reading frame with the NLS-Cre coding open reading frame. To finally maintain specific long term expression in the chick embryonic spinal cord three vectors were mixed and electroporated. The molar ratio was 5:1:1 for expression vector, transposase vector and Cre driver vector.

To create a versatile tool for the manipulation of the developing chick spinal cord several versions of the expression vector were created (Fig. 4.1). They are differing in the antibody recognition tag (V5 or FLAG), a signal sequence for the secretion pathway in the FLAG-TAG bearing vector and the expressed fluorescent marker proteins (eGFP, mGFP, Synaptophysin-GFP, mCherry (Chalfie et al. 1994, Okada et al. 1999, Venkatesh \& Murthy 2001, Shaner et al. 2004)).

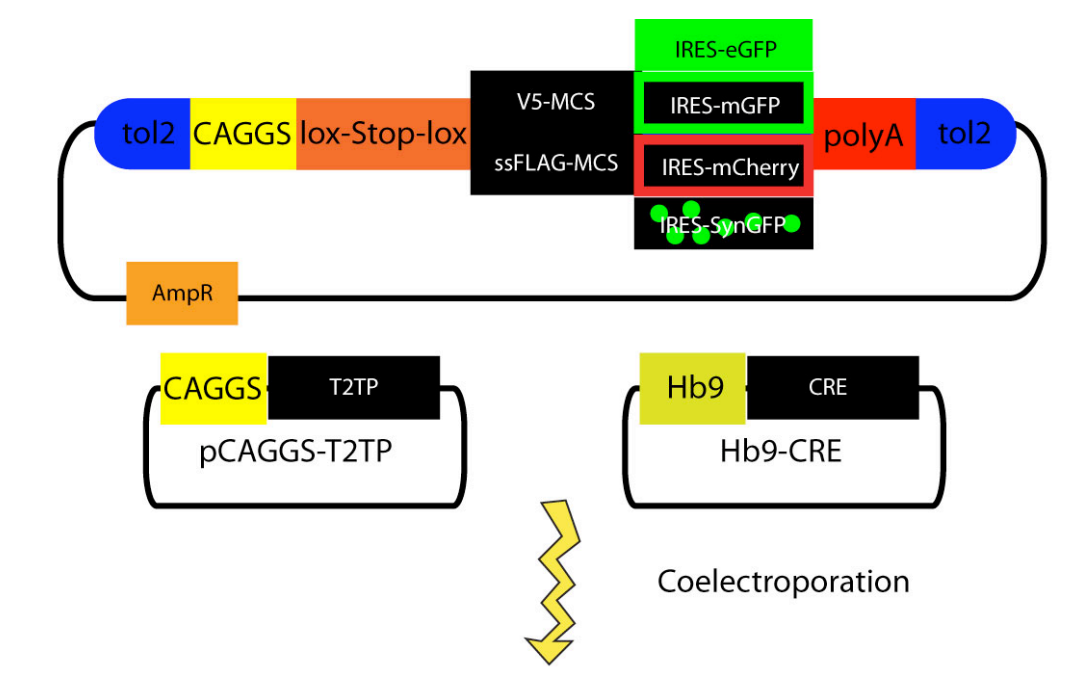

Stable genomic integration and motor neurons specific transgene expression

Fig. 4.1 The long-term stable expression system. The gene of interest was cloned into the expression vector and coelectroporated with the Cre driver construct and the tol2 transposase expression vector. The transposase recognizes the tol2 flanking sequences and integrates the expression cassette stably into the genome. At the same time Cre is expressed in motor neurons only and is activating transgene expression by excision of the lox-Stop-lox cassette. 
4. Methods

\subsection{Electrophysiology}

Patch-clamp recordings to evaluate the electrophysiological properties of chick MNs were performed in E12-E14 chick embryos. The embryos were electroporated on embryonic day 3 with either the eGFP expressing long-term expression vector or the long-term expression vector bearing a chick Dlk1-GFP fusion protein in combination with the pGK-Cre and the pCAGGS-T2TP vector.

Patch-clamp electrophysiological recordings have been performed by Chor Hoon Poh in our lab.

\subsection{Statistical analysis}

Error bars are indicating the standard deviation (SD) if not otherwise stated. Significance was calculated using Students t-test with two samples and unequal variance, unless otherwise stated. 


\section{Results}

\section{Results}

\subsection{Screening FMNT populations}

As the different FMNT populations were hard to access due to the lack of established markers the projects initial aim was to identify such genetic markers associated with fast and slow MNs. Thus, it was necessary to design an assay that allows the analysis of the differential gene expression profile of distinct FMNTs. In order to access the largest possible number of differential expressed genes with a limited amount of starting material a microarray based gene expression analysis was the method of choice. However, it was also necessary to access a pure or enriched population of a distinct FMNT. As already mentioned fast and slow FMNTs posses different cell soma sizes, so one possibility would lie in the enrichment of motor neurons of certain sizes via cell sorting. However, through this the cells would have to suffer from an axotomy and would have to be released from the tissue by a proteolytic digestion. Indeed, both processes would compromise the transcriptional profile and the integrity of the RNA for subsequent analysis. Moreover it is not clear how a distinct cutoff value for a distinct FMNT or MN class could be defined. The same problem would occur in a cell size based single cell laser capture based approach.

As part of an alternative approach, two mouse hind limb muscles showing a sharp difference in fibre-type composition were selected. Hence, the associated motor pools innervating these muscles are predicted to display a sharp difference in their FMNT composition (Fig. 5.1). These two specialized muscles are the Rectus femoris muscle that consists almost completely of fast type $2 \mathrm{~b}$ muscle fibres and the Soleus muscle, consisting virtually entirely of slow type 1 fibres, respectively (Kato et al. 1988, Wigston \& English 1992, Burkholder et al. 1994, Lu et al. 1999). Both muscles are hindlimb muscles. Thus, both motor pools are located within the LMC and overall motor column-specific gene expression differences are excluded. 


\section{Results}

A

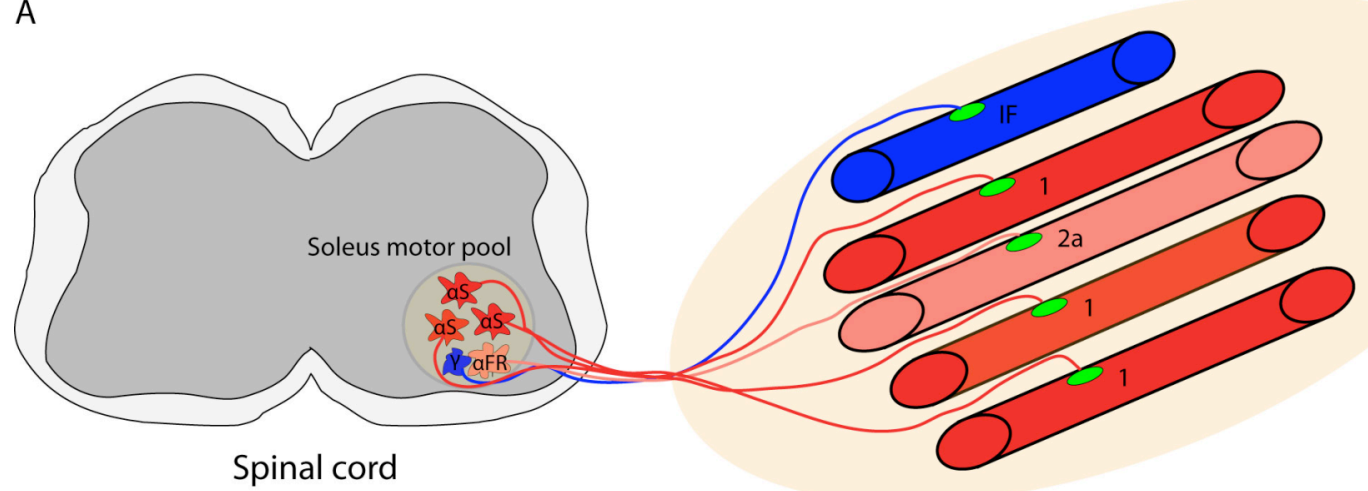

Soleus muscle

B

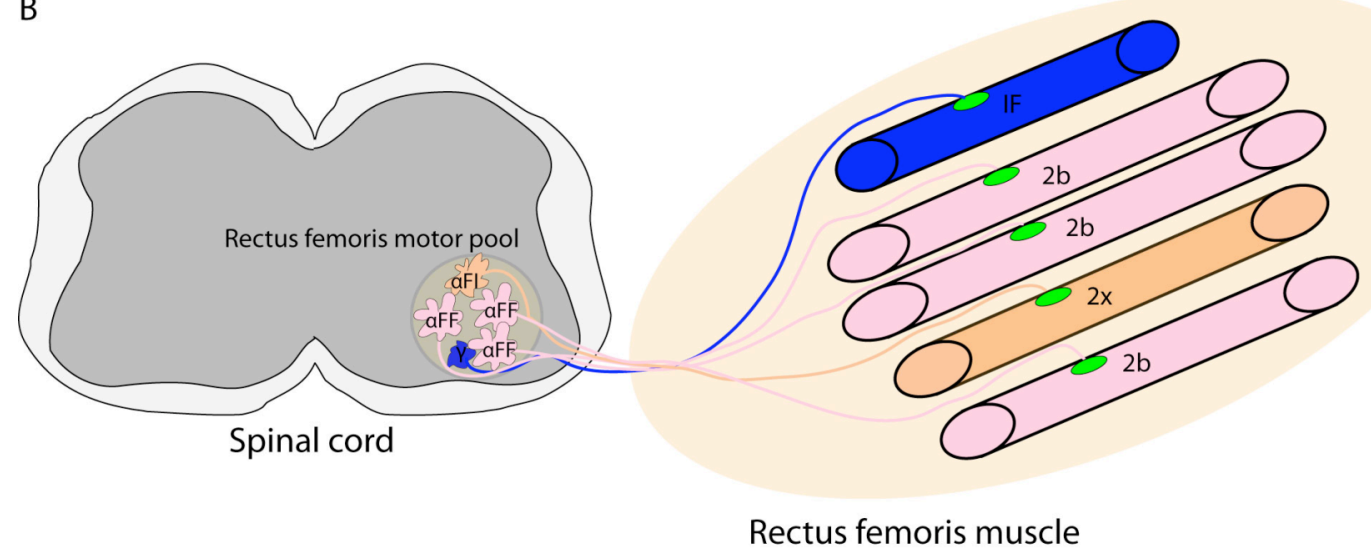

Fig. 5.1 Schematic of the Soleus and the Rectus femoris muscle and motor pool While the Soleus muscle (A) consists almost exclusively of slow muscle fibers, the Rectus femoris muscle (B) is consisting virtually entirely of fast fibers. Therefore the associated motor pools are also either predominantly consisting of slow or fast motor neurons.

In order to identify the MNs associated to these muscles it was necessary to find an appropriate retrograde tracing agent. This reagent must not affect the gene expression profile of the labeled cells, should be transported quickly through the axons and should show a bright fluorescent signal. So far, various agents have been used in order to retrogradely label cells in the central nervous system. Among these reagents are organic dyes like DiI and $\mathrm{DiO}$, the polysaccharide dextran and peptides like horseradish peroxidase as well as cholera toxin (Kristensson \& Olsson 1971, Snow et al. 1976, Stoekkel 1977, Robertson \& Grant 1985, Honig \& Hume 1986, Honig \& Hume 1989, Rajakumar 1993). For this work cholera toxin B, the non-toxin subunit of cholera toxin was selected due to its excellent labeling efficiency that was found to be superior to Alexa conjugated dextrans (data not shown). 


\section{Results}
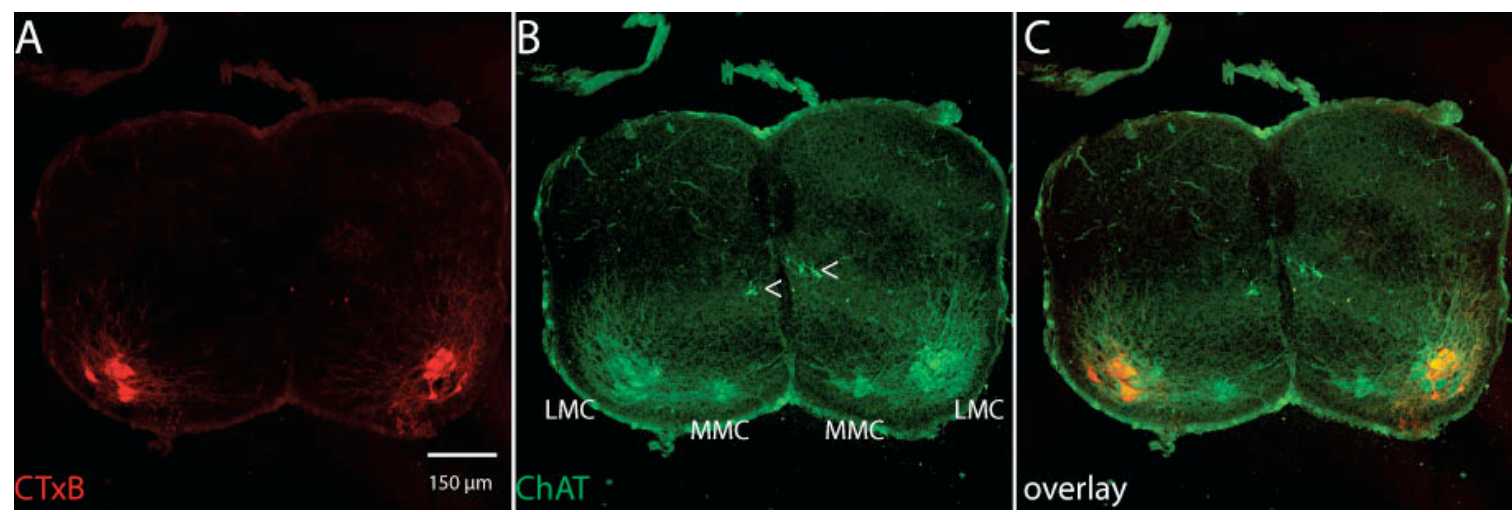

Fig. 5.2 Retrograde traced quadriceps motor pools Alexa-555 conjugated $\mathrm{CT}$ TB in the spinal cord after injection into the quadriceps muscles (A). Immunostaining for choline acetyltransferase (ChAT). ChAT labels motor neurons in lateral motor column (LMC), medial motor column (MMC) and cholinergic interneurons (arrowheads) (B). Overlay picture (C).

After injection into the muscle Alexa-conjugated cholera toxin $\mathrm{B}$ is thought to associate with lipid raft constituent sphingolipids and is transported effectively via the motor axons into the spinal cord (Fujinaga et al. 2003). There, a bright fluorescent signal could be observed in whole mount spinal cord preparations. After cryosectioning the signal could be observed exclusively in motor neurons within the spinal cord (Fig. 5.2). Outside the spinal cord, sensory neurons within the dorsal root ganglia could also be co-traced as CtxB is also transported via sensory axons (Wang \& Marquardt, unpublished). The fluorescence was still detectable eight hours post injection and persisted for at least 18 days according to the observation made in this work.

In order to access the associated MNs, the two muscles were injected with the fluorescently labeled tracer cholera toxin B subunit and retrogradely traced for two days post injection. The labeled motor pools were extracted via fluorescence-aided microdissection and the RNA was isolated from the cells. The RNA was then subsequently processed for microarray hybridization by Illumina BeadChip microarrays. The relative mRNA expression levels could be detected and were then analyzed for differentially expressed genes (Fig. 5.3). 


\section{Results}

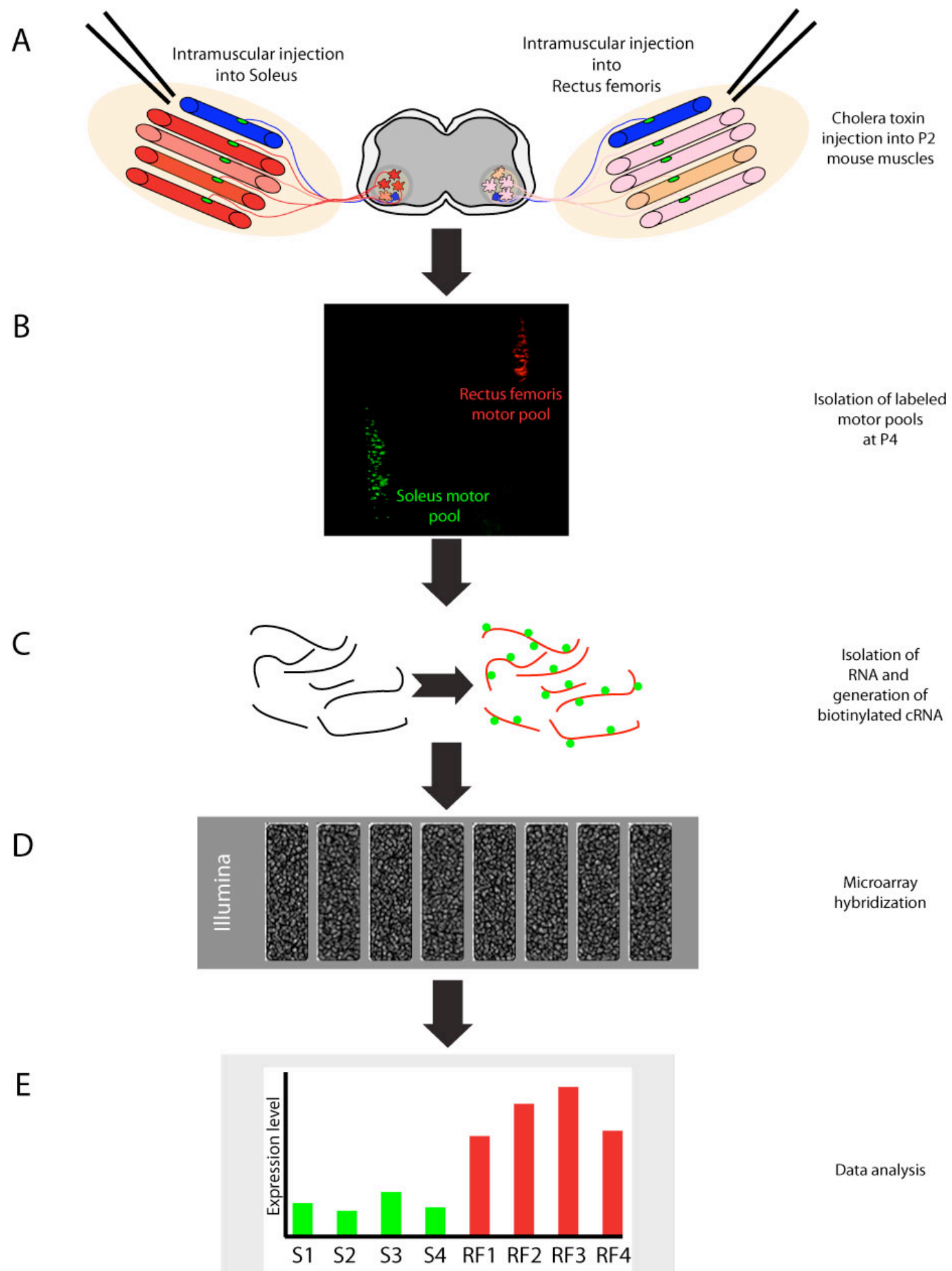

Fig. 5.3 Screen of fast and slow FMNT populations At P2 stage the slow muscle fiber enriched Soleus and the fast fiber enriched Rectus femoris muscle are injected with Alexa-conjugated cholera toxin B (A). Two days later the retrogradely traced motor pools are withdrawn from the spinal cord (B). The RNA is extracted and used as template for generation of biotinylated cRNA (C). This cRNA or aRNA is hybridized to Illumina BeadChip microarrays (D) and the expression levels of different genes are analyzed (E). 


\section{Results}

\subsection{Quantitative Expression analysis of FMNT populations}

After verifying the efficiency of the retrograde tracer $\mathrm{CTxB}$, fluorescence-aided microdissection was used to extract the labeled Rectus femoris and Soleus motor pools. Subsequently the RNA was isolated and processed for microarray hybridization. Selected genes differentially expressed according to microarray analysis were verified by quantitative real-time PCR.

\subsubsection{Microarray analysis}

A

Gene

Nxph2

Nts

Cbln4

Ly6a

Alcam

Alk

BC027127

Hoxd10

Mia1

Mmp9

Calb2

Ntng1

Sdk1

Npy

2700050C12Rik

2900093B09Rik

Zfp537

LOC434147

D12Ertd647e

Mfge8

B

Gene

Cart

Pvalb

Dlk1

Crabp1

6330527006Rik

Cdh8

Gna14

Actb

Pdrg1

Tmem14c

Siat7e

BC035954

Pappa

Amn

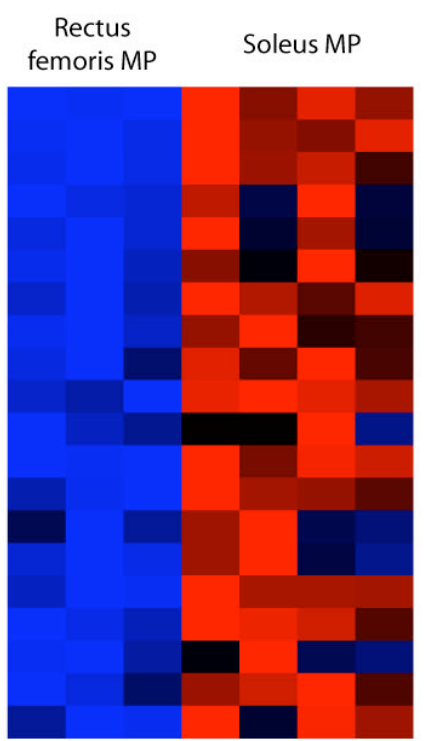

fold
change significance

$9,44 \quad * * *$

$9,14 \quad * * *$

$7,53 \quad * *$

$5,74 \quad *$

4,03

3,65

3,49

3,41

3,34

3,24

2,99

2,91

2,69

2,52

2,45

$2,17 \quad * * *$

$2,12 *$

$2,02 \quad * *$

$2,02 \quad * *$

$* * *$

$* *$

$* *$

$*$

$* * *$

$* * *$

$*$

**

$* *$

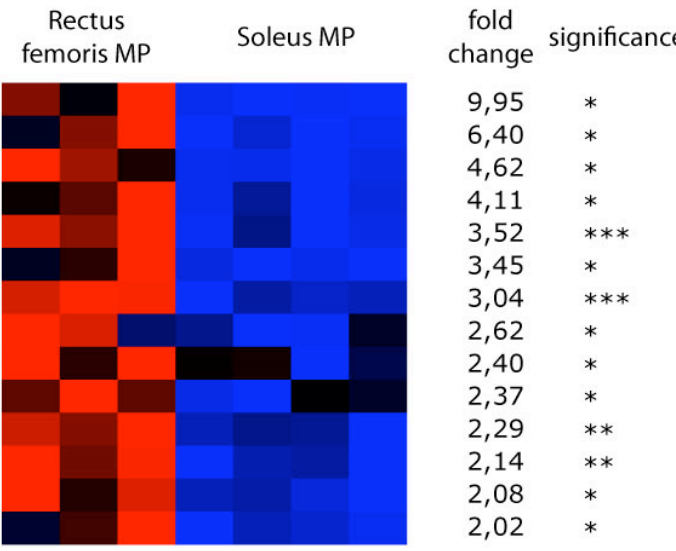

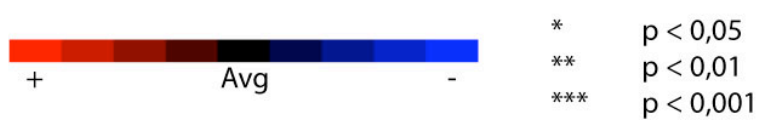

Fig. 5.4 Expression analysis of slow and fast FMNT enriched motor pools Heatmap of selected genes significantly upregulated in the slow $M N(n=4)$ enriched Soleus motor pool (A) or the fast $\mathrm{MN}(\mathrm{n}=3)$ enriched Rectus femoris motor pool (B). The threshold level fore gene expression was set to 100 relative units to avoid false positive results. 


\section{Results}
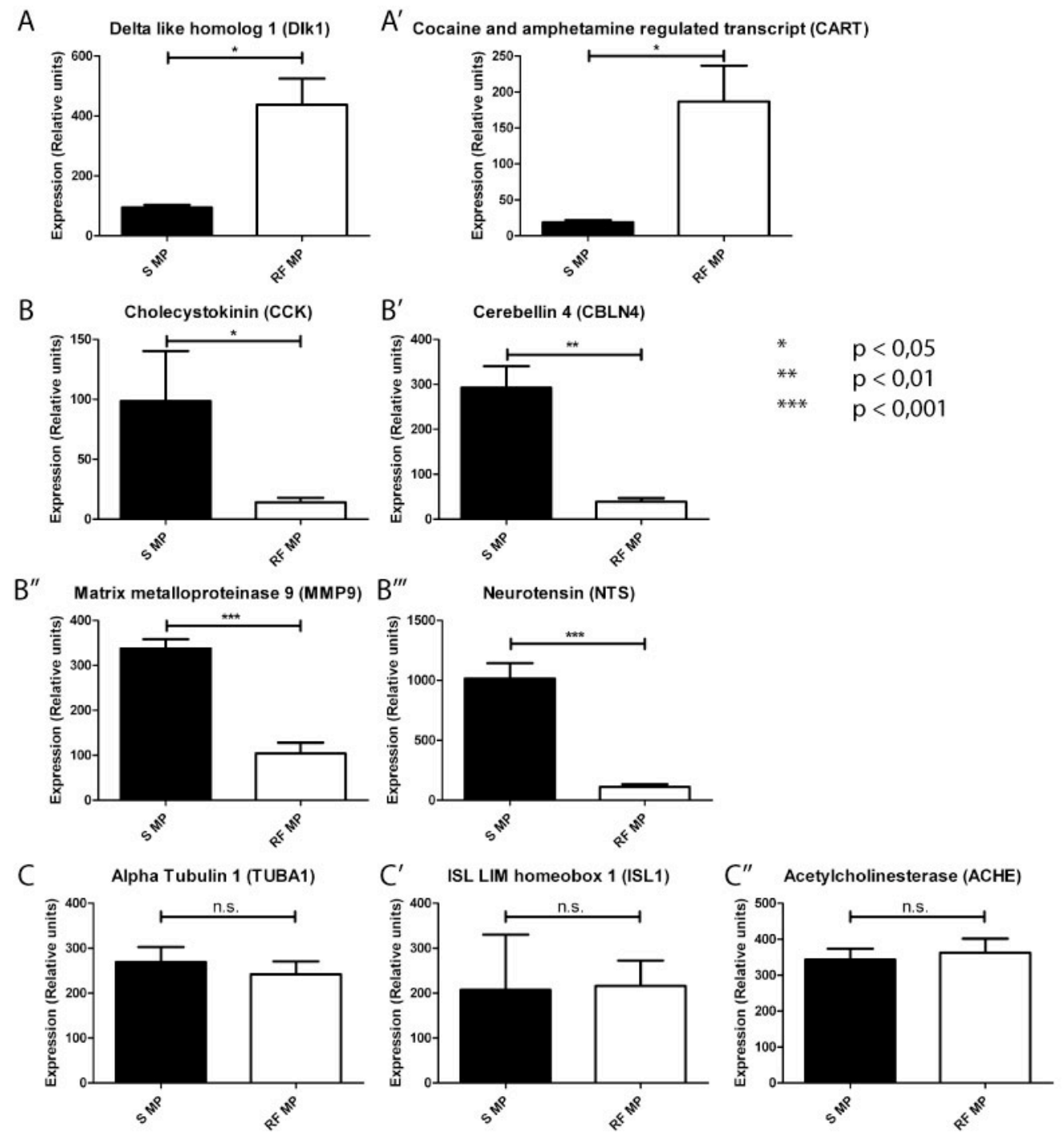

Fig. 5.5 Comparision of expression levels of selected genes Comparision of expression levels of genes differentially regulated in between different motor pools. The Dlk1 (A) and the Cart gene (A') are upregulated in the Rectus femoris motor pool (RF MP), while Cck (B), Cbln4 (B'), Mmp9 (B'') and Nts (B',') genes are upregulated in the Soleus motor pool (S MP). The general housekeeping gene Tubal (C) as well as the general MN marker genes Isl1 (C') and AchE (C',) are shown as controls.

Microarray analysis of the two motor neuron populations revealed a set of differentially expressed genes putatively associated with individual FMNTs. However, it is necessary to discriminate between genes that are linked either to a specific motor pool or are differentially expressed in between shank and thigh innervating MNs and actually FMNT specific genes. To be considered as a FMNT specific, a gene has to fulfill certain criteria. It must not be expressed within all MNs, but in a "salt and pepper" fashion instead. Also such a gene has to be present at all rostrocaudal levels and all motor columns. Furthermore the expression must not be 


\section{Results}

restricted towards certain motor pools. Hoxd10 and Cadherin 8 do not fulfill these requirements. Both genes are expressed in a motor pool specific fashion and contribute to motor pool specification (Price et al. 2002, Dasen et al. 2005, Dalla Torre di Sanguinetto 2008). Thus, these genes have been excluded from the further analysis.

\subsubsection{Data verification by quantitative real-time PCR}

As the microarray experiment has shown several candidate genes for different FMNTs it was necessary to verify their expression. An initial indication was given by the in-situ data from the Allen Brain Atlas (Lein et al. 2007, www.brain-map.org). A MN expression resembling a "salt and pepper" pattern was expected for a putative FMNT specific gene. However, as several probes and sections published in this high throughput screen did not show a proper staining and it was impossible to make a qualified statement about FMNT specificity according to the published pictures. Hence, quantitative real-time PCR experiments from independent motor pool samples were run in order to ascertain the differential expression between the different $\mathrm{MN}$ groups.

A

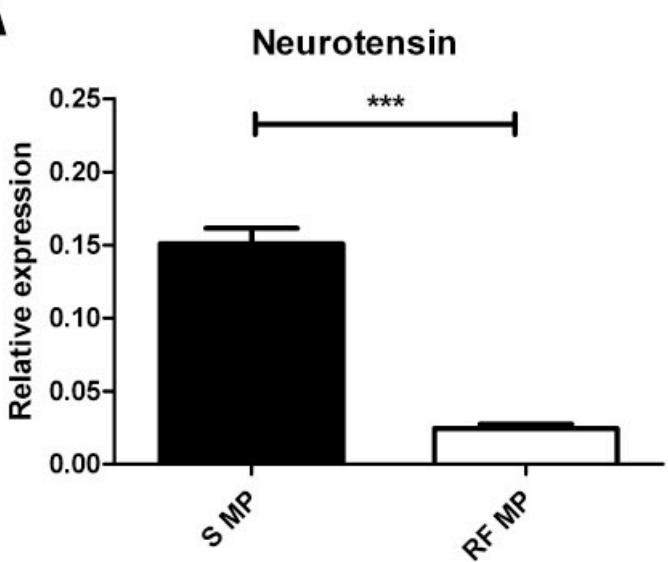

B

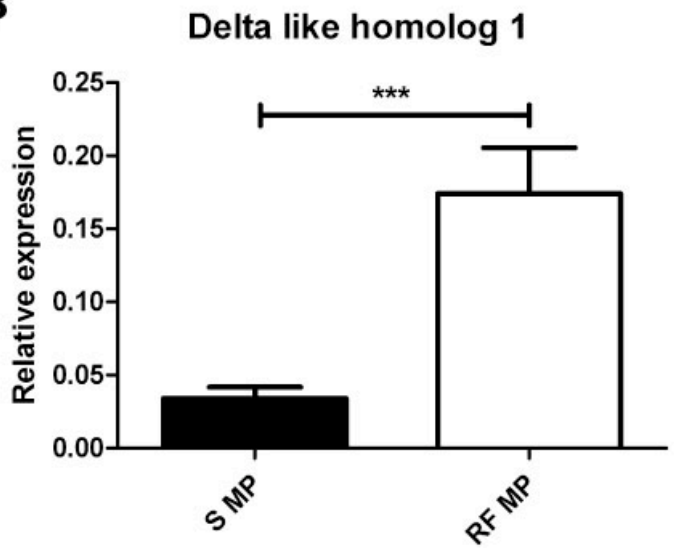

Fig. 5.6 Quantitative real-time PCR analysis of Neurotensin and Dlk1 expression To verifiy the microarray results suggesting a differential expression of Neurotensin (A) and Dlk1 (B) between Soleus (S MP) and Rectus femoris (RF MP) motor pool the gene expression was analysed by qPCR $(n=3)$. Expression level of the alpha tubulin 1 $\mathrm{B}$ mRNA was claimed as 1 . The expression levels of Nts and Dlk1 were normalized to the expression of that protein.

The results obtained by quantitative real-time PCR analysis have proven the findings from the microarray experiment. Hence, Dlk1 and Nts (Neurotensin) have proven to 


\section{Results}

be expressed differentially in between the two MN populations. However, the Nts expression was found to be shank/thigh specific after subsequent experiments (data not shown). In the end Dlk1 was the most promising candidate for FMNT specific expression and was analyzed in the further experiments. It was chosen, since it is suggested to be involved in the Notch pathway one of the pivotal developmental pathways and also is known to influence cell fate decision in preadipocytes (Smas et al. 1993, Smas et al. 1997, Baladron et al. 2005, Bray et al. 2008). These considerations make Dlk1 the most promising factor for a role in FMNT specification and development.

\subsection{Qualitative Dlk1 expression analysis}

As mentioned before a gene has to fulfill several criteria in order to be considered as FMNT specific marker. Hence, the expression of Dlk1 was analyzed in combination with different motor neuron markers as well as at different embryonic and postnatal stages. Furthermore the expression pattern at all rostrocaudal levels and within different motor columns and several retrograde labeled motor pools was assessed.

\subsubsection{Presence of Dlk1 expression at different rostrocaudal levels}

We next verified if Dlk1 is a marker gene specific for a distinct FMNT, but not for a specific motor pool or column like it has been shown for several of the Hox cluster or cadherin genes. Thus, the expression of Dlk1 was analyzed in brachial, thoracic and lumbar spinal cord levels. For this the presence of the specific mRNA and the Dlk1 protein was examined via in-situ hybridization and immunostaining (Fig. 5.7 and Fig. 5.8).

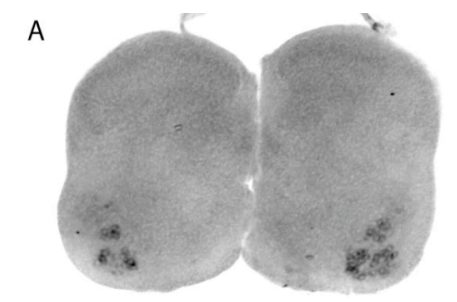

brachial
B

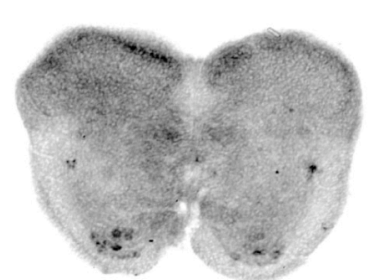

thoracic

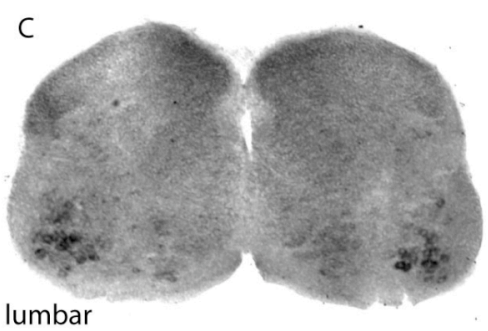

lumbar

Fig. 5.7 In-situ analysis of Dlk1 expression at different rostrocaudal levels at P4 In-situ expression pattern of Dlk1 at lumbar (A), thoracic (B) and brachial (C) level.

As indicated by the in-situ hybridization analysis the gene was actively transcribed within large soma size cell within the ventral horn of the mouse spinal cord at 


\section{Results}

postnatal day 4 . The mRNA could be detected within all rostrocaudal levels analyzed. Both cell size and position of the Dlk1 positive cells indicated that these cells are motor neurons.
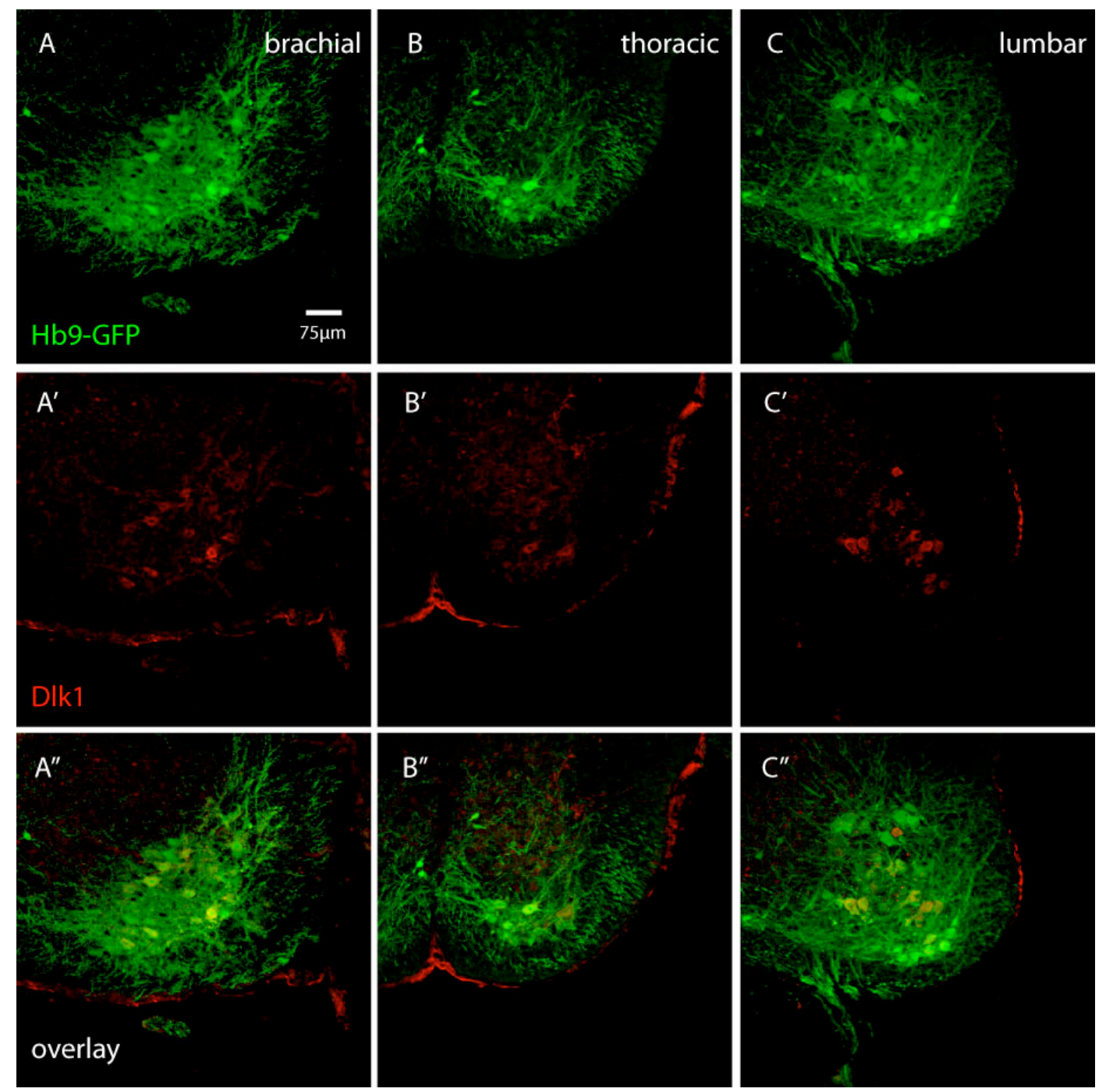

Fig. 5.8 Immunohistological analysis of Dlk1 expression at different rostrocaudal levels in Hb9-GFP transgenic mice at P4 Hb9-GFP expression transgenetically marks postmitotic motor neurons (A, B, C), Dlk1 immunostaining (A', B', C'), overlay picture (A', B', C',).

The complementary analysis was performed using Hb9-GFP transgenic mice (Lee et al. 2004) in order to correlate the presence of Dlk1 with the motor neuron cell identity. Expression of the Hb9-GFP transgene is broadly used to identifiy motor neurons within early developmental stages up to P15 (Betley et al. 2009). As expected the cells positively labeled with the anti-Dlk1 antibody positive within the ventral horn of the spinal could be linked to Hb9-GFP transgene expressing cells. Hence, Dlk1 is expressed in a subset of MNs, but not in a motor pool-specific or regionspecific manner. 


\section{Results}

\subsubsection{Dlk1 expression in different motor columns}

In order to further investigate the expression of Dlk1 within different motor columns the expression in the lumbar MMC and the lumbar LMC was examined. At lumbar of brachial levels these two columns can easily differentiated spatially, thus correlation with a column specific marker is not necessary.
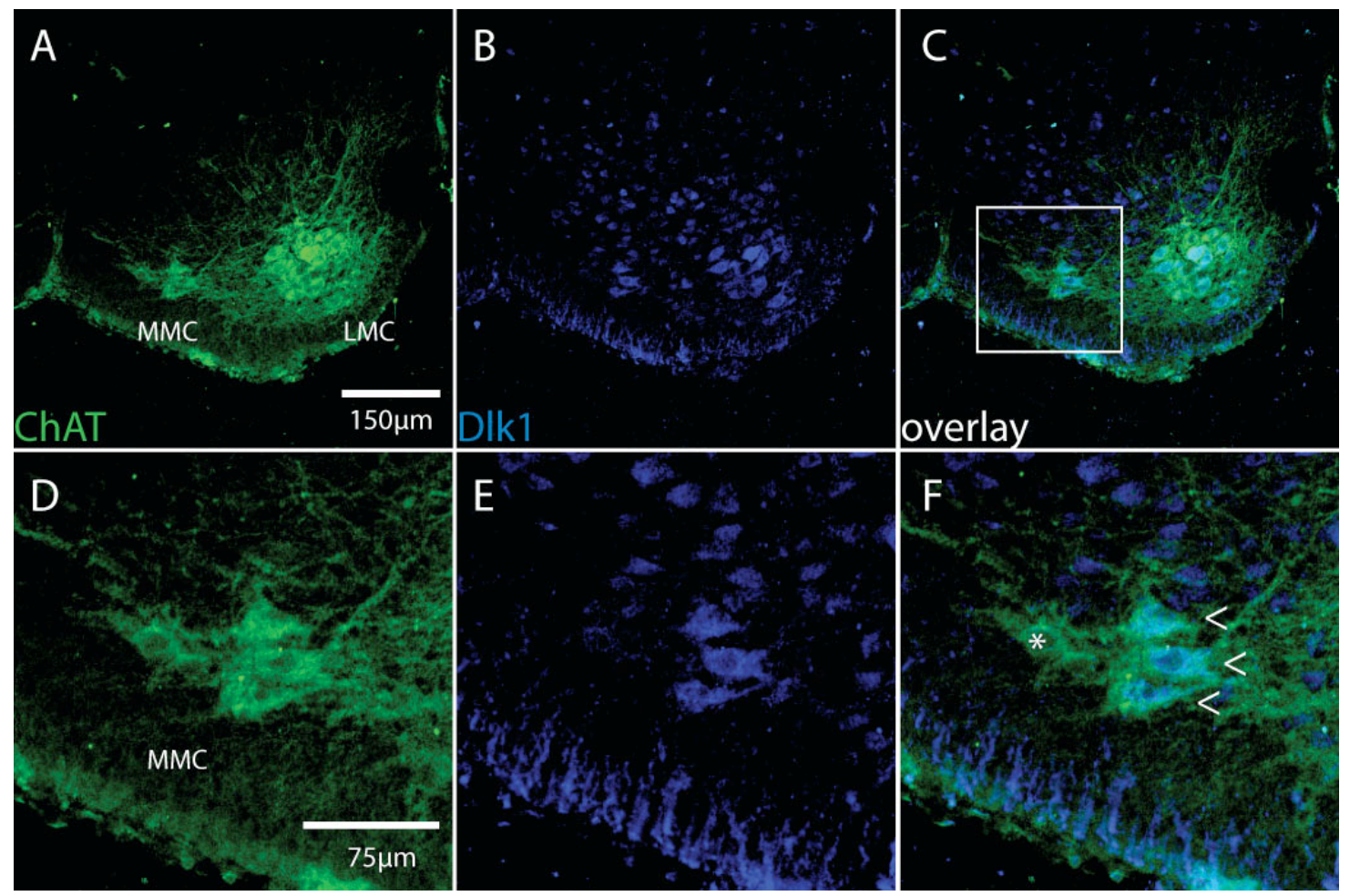

Fig. 5.9 Dlk1 expression in LMC and MMC Immunostaining for ChAT and Dlk1 on P4 lumbar mouse spinal cords. Expression of Dlk1 is shown in LMC and MMC (A-C). Inset shows higher magnification of the MMC (D-F). Dlk1 positive (arrowheads) as well as negative cells (asterisk) were detectable.

In general these results show Dlk1 to be expressed independently of the rostrocaudal level, as well as motor column identity, thereby fulfilling key requirements for an FMNT-specific expressed gene.

\subsubsection{Analysis of Dlk1 expression in alpha and gamma motor neurons}

Subsequently it was determined whether the expression of Dlk1 in a subset of MN is associated with a specific MN class. Alpha MNs are larger in cell soma size compared to gamma MNs and innervate the extrafusal muscle fibers while the gamma MNs are innervating the intrafusal muscle fibers in the muscle spindles. The gamma MNs make up approximately one third of the entire MN population (Kuffler et al. 1951, Friese et al. 2009, Shneider et al. 2009). 


\section{Results}
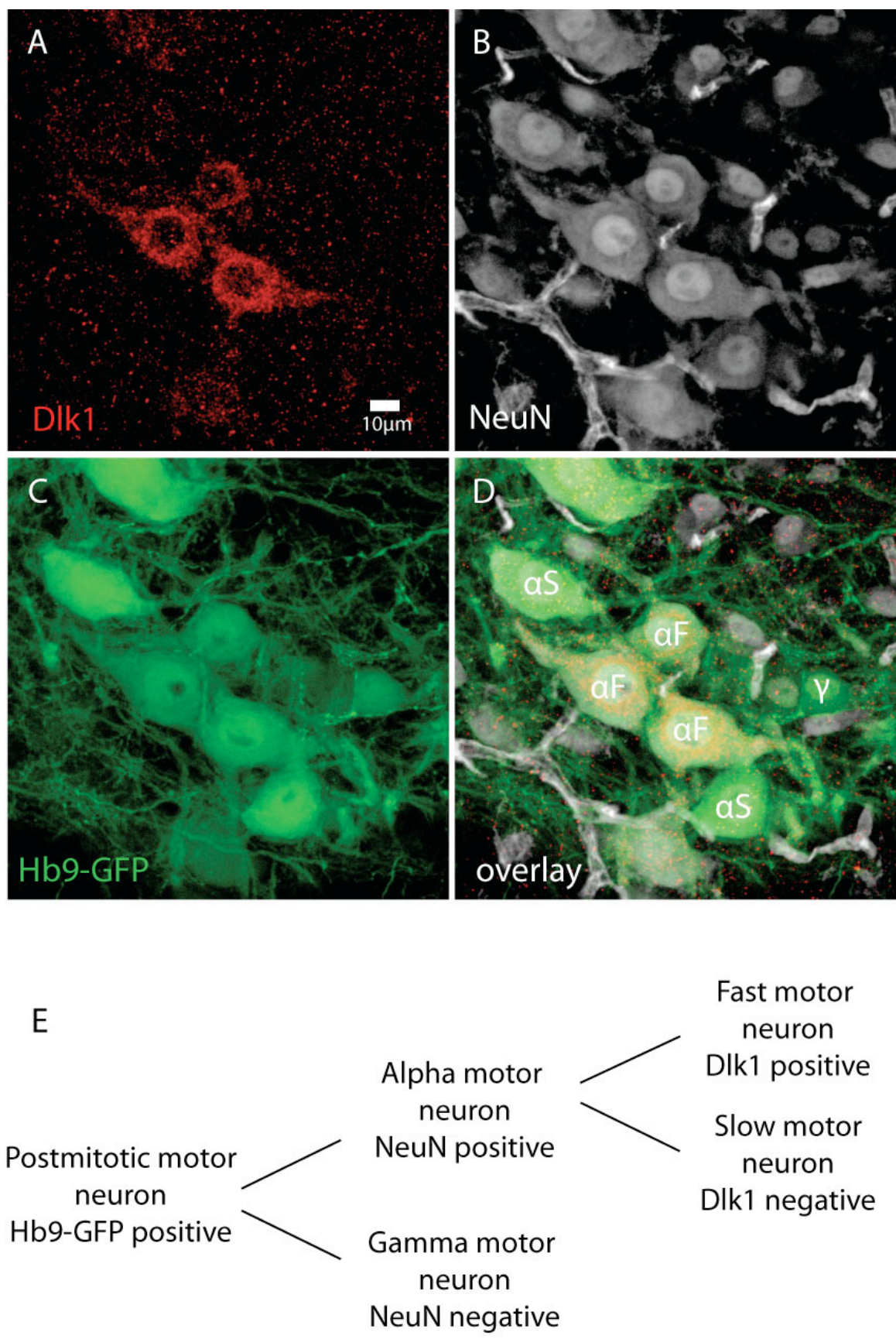

Fig. 5.10 Dlk1 expression in $\boldsymbol{\alpha}$ motor neurons at P4 Staining for Dlk1 (A), NeuN (B), Hb9-GFP (C) and overlay picture (D). Schematic overview of MN class and FMNT identity and associated genetic markers (E). Dlk1 expression is detectable within a subset of alpha MNs.

Dlk1 was detected in a population of Hb9-GFP positive cells. As already mentioned expression of the Hb9-GFP transgene marks MNs up to P15. After counterstaining with the alpha MN marker NeuN the gamma MNs could be distinguished by the weak expression of the protein that was restricted exclusively to the nucleus of the $\mathrm{Hb} 9$ - 


\section{Results}

GFP positive cells (Friese et al. 2009). In contrast, alpha MNs typically exhibit not only a strong nuclear NeuN staining but also a clear cytosolic staining. Expression of Dlk1 was detectable only within some of Hb9-GFP positive cells that show a strong staining for NeuN but not in NeuN negative/NeuN weak cells. Thus, it is specifically expressed within alpha MNs. As not all alpha MNs were found to be stained it was concluded that Dlk1 gene expression at early postnatal stages is restricted towards a discrete subpopulation of alpha MNs.

\subsubsection{Characterization of Dlk1 expression in different motor pools}

Next, it was tested whether the expression of Dlk1 in a subset of alpha MNs was consistent with an expression in a specific type of FMNTs. To achieve this four exemplary motor pools innervating predominantly fast or slow muscles were retrogradely traced and examined for Dlk1 expression. Moreover these muscles represent different areas regions of the skeletal musculature. The Soleus and Rectus femoris motor pools were examined, as they have been initial targets in the microarray screen and should show a completely different expression pattern of the Dlk1 gene. The Tibialis cranialis muscle (termed Tibialis anterior in human) is a shank muscle as the Soleus, but enriched in fast muscle fibers. Thus, a shank/thigh specific expression profile of Dlk1 could be examined. The Triceps brachii however represents the forelimb musculature and contains only a low amount of slow type 1 muscle fibers, although its muscle fiber distribution is region specific (Fuentes et al. 1998, Matsumoto et al. 2007). 


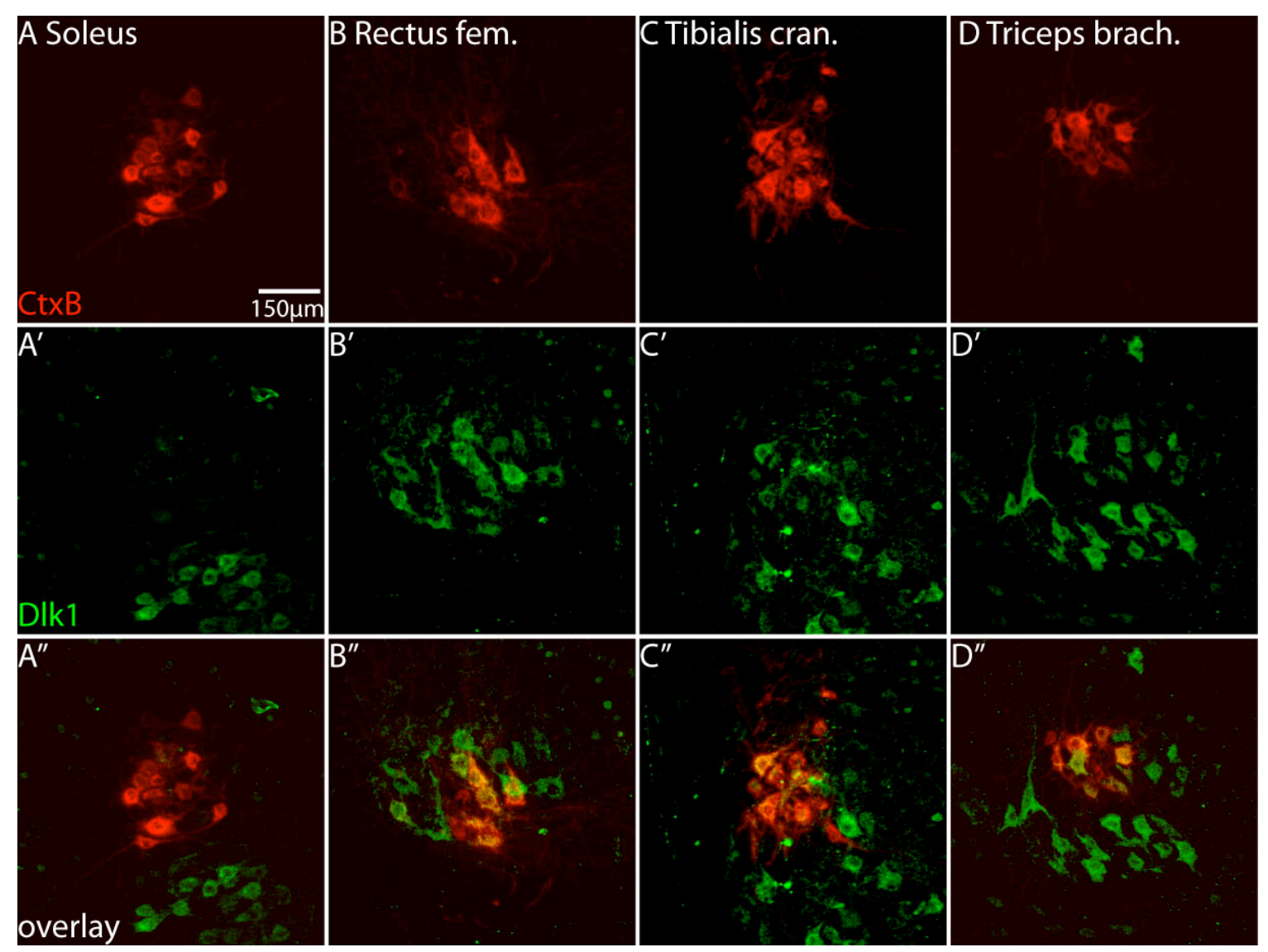

Fig. 5.11 Dlk1 expression in different motor pools Retrograde tracing of Soleus (A), Rectus femoris (B), Tibialis cranialis (C) and Triceps brachii (D) motor pools with cholera toxin B. Immunostaining for Dlk1 (A', B', C' and D'). Overlay pictures (A', B', C', and D').

The expression pattern obtained was consistent with the microarray and qPCR data. Only very few cells within the slow MN enriched Soleus motor pool were expressing Dlk1 while it was found to be expressed in a widespread fashion within the other motor pools. Therefore, the Dlk1 expression in motor pools correlates with the muscle fiber composition of the associated muscle on a qualitative level.

Dlk1 expression was found to concur with muscle fiber proportion in innervated target muscles in a qualitative fashion. To substantiate this finding the expression in the previously examined motor pools was quantified. Therefore the Soleus, the Rectus femoris, the Tibialis cranialis and the Triceps brachii muscles of P2 mice were bilaterally injected with cholera toxin and retrogradely traced. After the animals were sacrificed at P4 the spinal cord was dissected and cryosection were performed of the labeled regions. The sections were subsequently stained with the anti-Dlk1 antibody and the staining was correlated with retrogradely traced cells (Fig. 5.12). The same experiment was performed for P10 animals in order to monitor if there are any 


\section{Results}

changes in the Dlk1 expression pattern at later postnatal stages. In order to monitor possible changes in Dlk1 expression in later developmental stages the experiment was repeated with retrogradely traced Soleus and Rectus femoris MNs at P10 (Fig. 5.13).

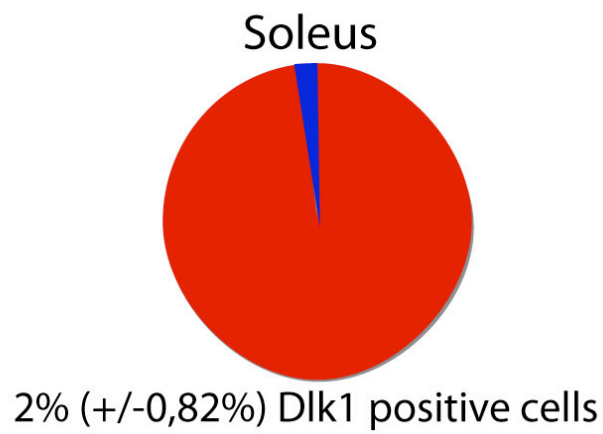

Tibialis cranialis

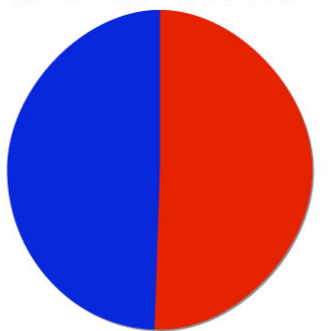

49,33\% (+/-2,05\%) Dlk1 positive cells

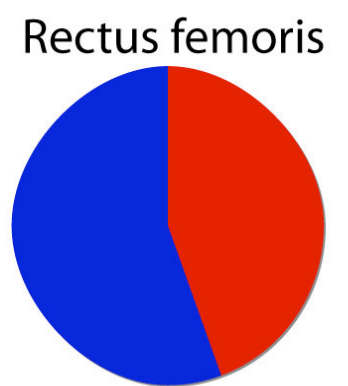

55,66\% (+/-4,02\%) Dlk1 positive cells

Fig. 5.12 Percentage of Dlk1 positive retrogradely labeled cells in different motor pools at P4 Dlk1 expression was quantified in motor pools retrogradly traced after muscle injection of cholera toxin B. Cells positive for cholera toxin B only are labeled in red, cells positive for Dlk1 immunostaining are shown in blue. 100 cells per animal three animals per pool were analyzed. The standard deviation (SD) is given in brackets.

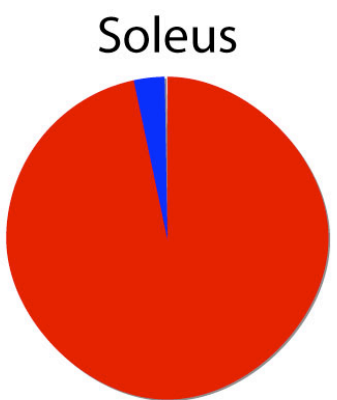

3,33\% (+/-0,47\%) Dlk1 positive cells

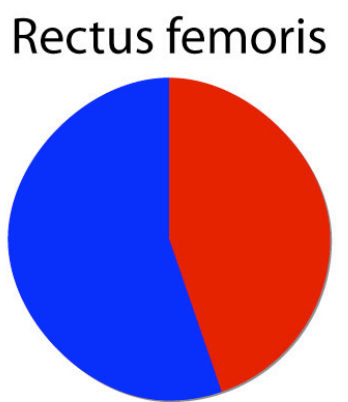

$55,33 \%(+/-1,70 \%)$ Dlk1 positive cells

Fig. 5.13 Percentage of Dlk1 positive retrogradely labeled cells in Soleus and Rectus femoris at P10 Dlk1 expression was quantified in the motor pools of Soleus and Rectus femoris. Cells positive for cholera toxin B only are labeled in red, cells positive for Dlk1 immunostaining are shown in blue. 100 cells per animal three animals per pool were analyzed. The standard deviation (SD) is given in brackets. 


\section{Results}

The expression of Dlk1 in the retrogradely traced motor pools concurred with the composition of muscle fibers of the associated muscles. While there were only very few Dlk1 positive cells detectable within the retrogradely labeled Soleus innervating motor pool, about $55 \%$ of all MNs of the Rectus femoris motor pool MNs were positively stained for the protein. Tibialis cranialis and Triceps brachii are also predominantly fast muscles according to the motor neuron structure. The Tibialis cranialis has previously been identified as a fast muscle by the results of Burkholder et al. (Burkholder et al. 1994). Also the proportion of Dlk1 positive cells seems to be constant during early postnatal development up to P10. Since the expression in MNs correlates with the muscle fiber composition of a given motor pool a fast FMNT specific expression of Dlk1 is strongly suggested.

\subsection{Cell soma size as a marker for different motor neuron types}

As Dlk1 has fulfilled all of the general criteria for a FMNT specific gene, it was crucial to confirm these findings according to another known FMNT-specific parameter. Therefore the MN cell soma size was chosen, since it is a commonly accepted and stringent criterion to discriminate between different MN populations (Webber \& Pleschka 1976, Burke et al. 1977, Kernell \& Zwaagstra, 1981, Ulfhake \& Kellerth 1982, Ishihara et al. 1988). To utilize the cell soma size as a marker for different motor neuron types, it has to be determined if the cell size of different FMNTs already differs at early stages of postnatal development. According to previous publications differentiation of different MN classes and FMNTs is possible also at early postnatal stages (Friese et al. 2009, Shneider et al. 2009 Enijin et al. 2010). To establish this assay, initially the motor neuron size and the cell size distribution for alpha and gamma motor neurons were analyzed in Hb9-GFP transgenic mice. Additionally, the cell soma sizes of motor neurons in distinct motor pools innervating different muscles were examined.

\subsubsection{Size determination of alpha and gamma motor neurons at $\mathbf{P 4}$}

To distinguish between alpha and gamma MNs cryosections of the lumbar spinal cord of stage P4 Hb9-GFP mice were stained with an anti-NeuN antibody. The size was determined at lumbar levels due to the high number of MNs at that region. For 


\section{Results}

practical reasons only the cell sizes of LMC MNs were quantified. It was possible to distinguish in between alpha and gamma MNs as both classes exhibit a clear difference in NeuN expression (Fig. 5.10). While alpha MNs show a strong cytosolic signal subsequent to of NeuN immunostaining, NeuN expression is absent or only weakly expressed in the nucleus of gamma MNs.

A

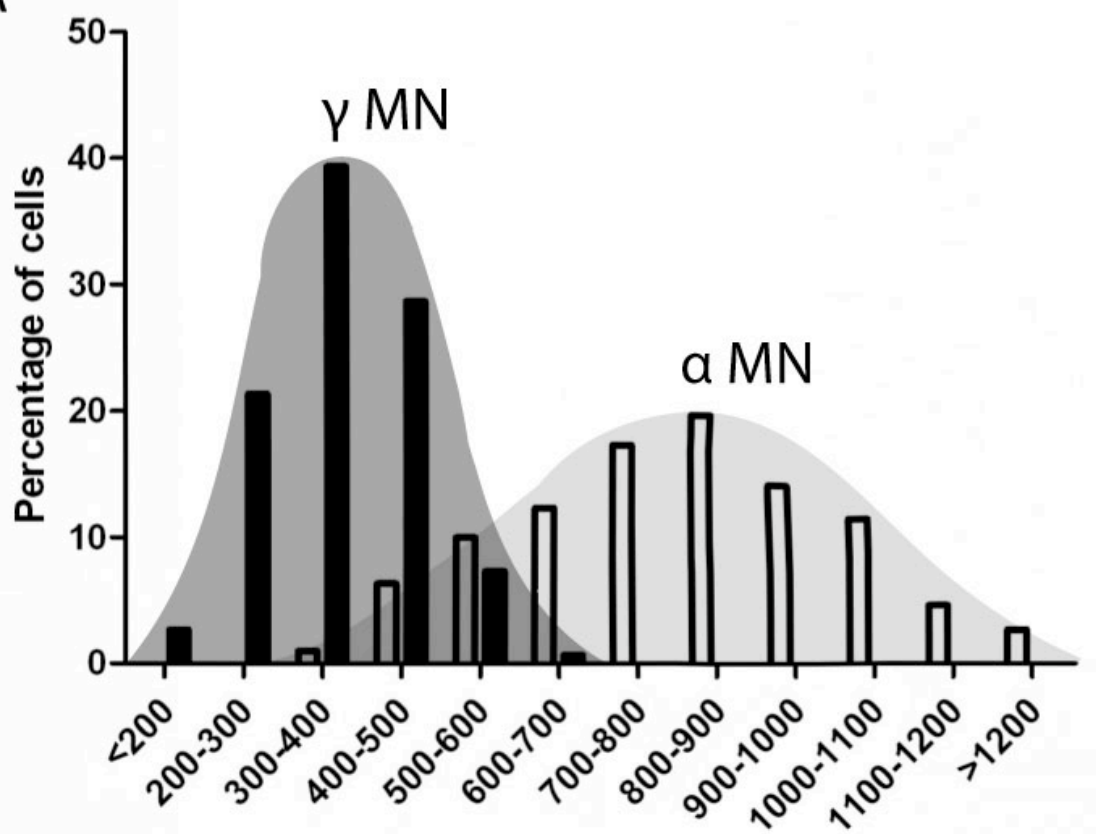

Cell size $\left(\mu \mathrm{m}^{2}\right)$

B

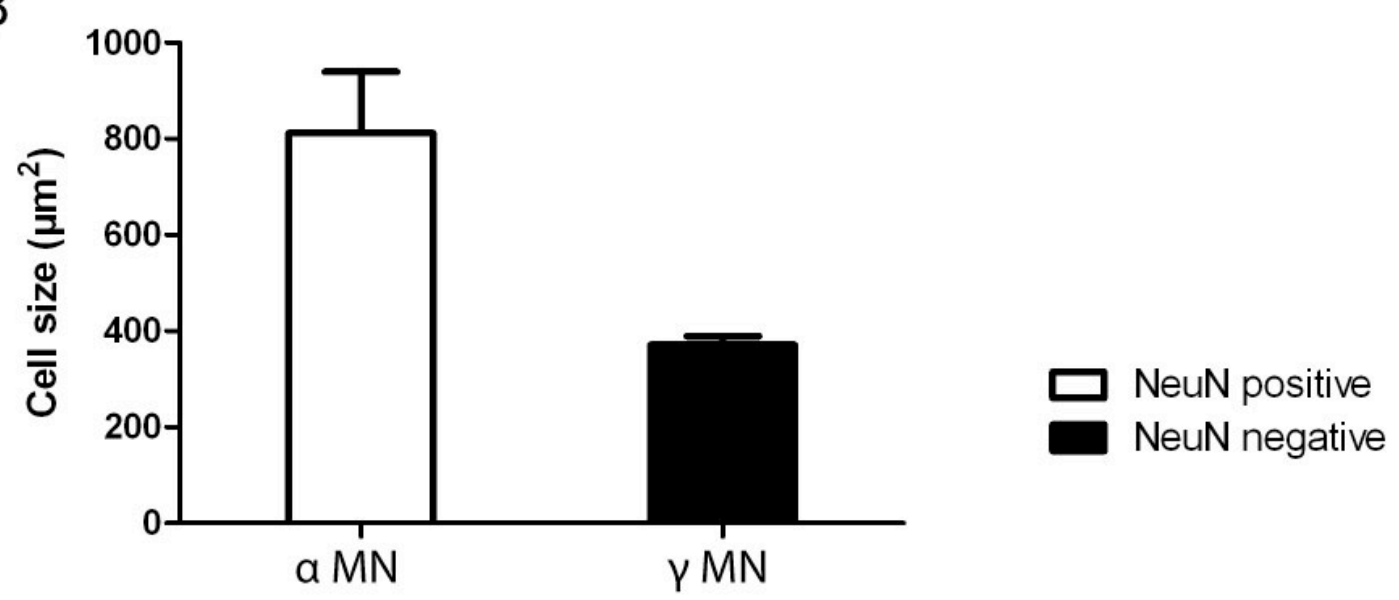

Fig. 5.14 Cell size distribution and average cell size of $\alpha$ and $\boldsymbol{\gamma}$ motor neurons at P4 Cell size distribution in percent of all NeuN positive and negative cells (A) Average cell size of NeuN positive and negative cells (B). $50 \mathrm{NeuN}$ negative cells from three animals were analyzed. For the NeuN positive population $50 \mathrm{Dlk} 1$ positive and 50 Dlk1 negative cells have been analyzed from three different animals $(n=3)$. 


\section{Results}

A clear difference in average cell size as well as in relative cell size distribution was detectable between NeuN positive alpha MNs and NeuN negative gamma MNs as early as postnatal day 4 . This further indicated that molecular differences in between these two motor neuron types are already established at the given stage. A reduction of GFP expression in NeuN negative cells could not be monitored contradicting the findings of Shneider et al. that gamma MNs do not express Hb9-GFP (Shneider et al. 2009). This might be explained be the use of different Hb9-GFP transgenic mouse lines. As for this work the Hb9-GFP mouse line described by Lee and colleagues was used while Shneider et al. have performed their experiments with the mouse line described by Wichterle et al. (Wichterle et al. 2002, Lee et al. 2004).

\subsubsection{Determination of cell size in different motor pools}

Since the previous results indicated that alpha and gamma MNs can be differentiated based on their cell soma sizes at early postnatal stages, it was determined if there are specific differences in cell soma sizes in between the MN populations of different motor pools. Therefore, the motor pools of Soleus, Rectus femoris, Tibialis cranialis and Triceps brachii were retrograde labeled and the cell soma size was measured according to the area labeled by $\mathrm{CTxB}$. The cell size differences were examined at P4 for these four motor pools and also at P10 for the Soleus and Rectus femoris motor pools in order to monitor cell growth. For all quantifications at least $100 \mathrm{MNs}$ from three bilaterally injected mice were evaluated $(n=3)$. 


\section{Results}
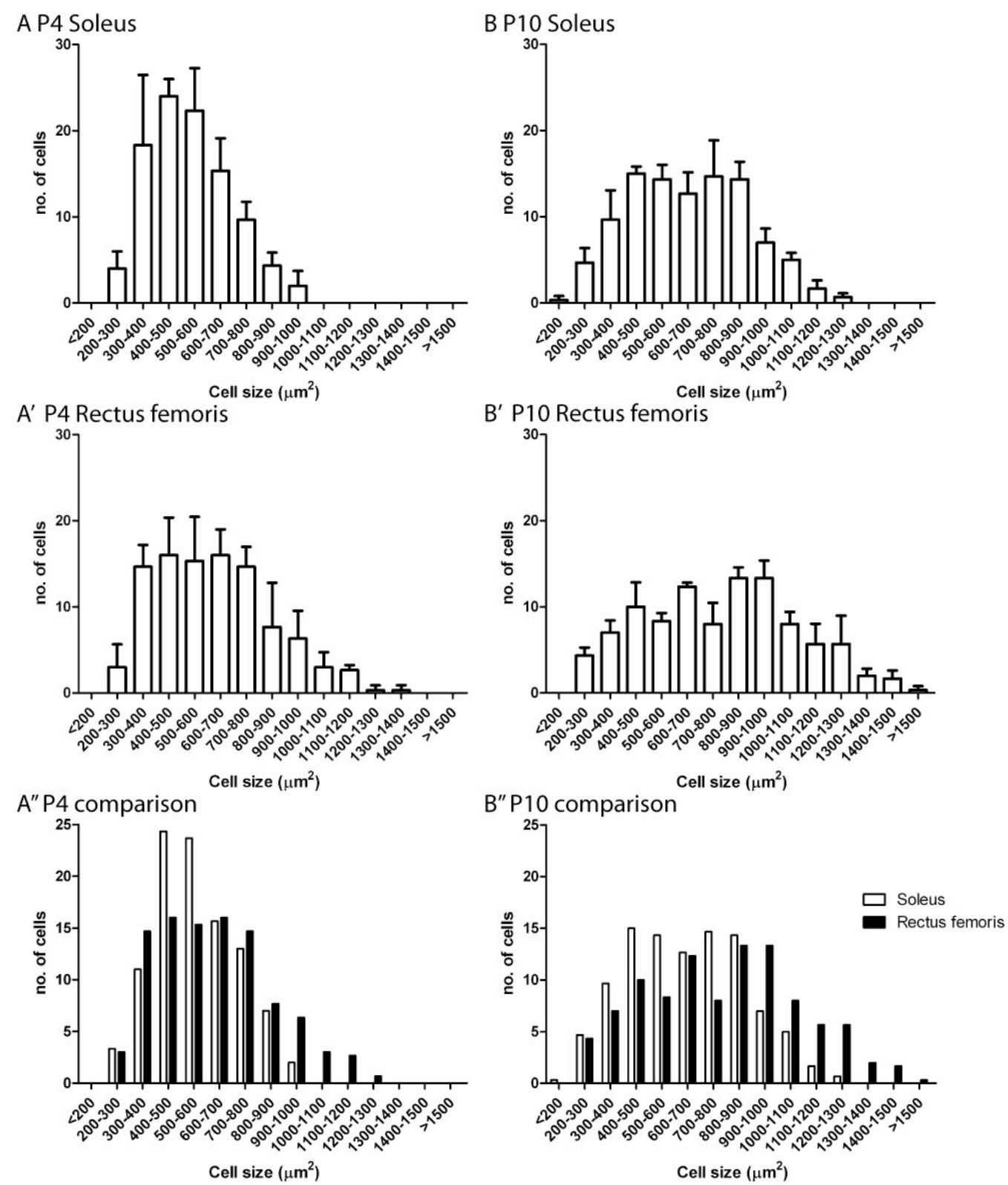

Fig. 5.15 Comparison of cell soma sizes of different motor pools at P4 Cell size distribution of retrogradly traced motor neurons in Rectus femoris (A), Soleus (B), Tibialis cranialis (C) and Triceps brachii (D) motor pool. Comparison of cell soma size distribution of Rectus femoris and Soleus motor pools $(E)(n=3)$.

A difference in the cells size distributions of the four different motor pools examined here could be shown. This difference is most clearly visible for the Rectus femoris and Soleus innervating MNs. While the MNs from the Soleus motor pool have an average size of $533 \mu \mathrm{m}^{2}$ ( $\mathrm{SD}+/-13.4 \mu \mathrm{m}^{2}$ ), the average Rectus femoris MN measured $628.1 \mu \mathrm{m}^{2}$ (SD $+/-47.4 \mu \mathrm{m}^{2}$ ). This resembles a size difference of $17.8 \%$. Thus, already at $\mathrm{P} 4$ stage significant differences $(\mathrm{p}<0.05)$ in average cell size in between the motor pools can be obtained. The Tibialis cranialis MNs had an average cell size of 


\section{Results}

$623.1 \mu \mathrm{m}^{2}\left(\mathrm{SD}+/-39.3 \mu \mathrm{m}^{2}\right)$ while that of Triceps brachii MNs was $561.2 \mu \mathrm{m}^{2}$ (SD $+/-31.8 \mu \mathrm{m}^{2}$ ). Thus, the differences obtained in cell soma size of different motor pools did not always strictly correspond to their expected FMNT composition. At P10 the size difference between Soleus and Rectus femoris MNs had increased to $19.1 \%$, the Soleus MN had an average soma size of $657.4 \mu \mathrm{m}^{2}$ (SD $+/-27.3 \mu \mathrm{m}^{2}$ ) compared to $783.2 \mu \mathrm{m}^{2}\left(\mathrm{SD}+/-9.7 \mu \mathrm{m}^{2}\right)$ for Rectus femoris MNs.

All calculations included the whole population of MNs that had been retrogradely traced including the gamma MNs with a very small soma size. Thus, another screen restricted to strongly NeuN expressing alpha MNs would yield a clearer result regarding the FMNT distribution.

\subsubsection{Cell soma size distribution of Dlk1 expressing cells}

Since the previous calculations were based upon the whole retrogradely labeled cell population these results are impaired by the presence of the gamma MNs within the motor pools. Gamma MNs are suggested to account for one third of the overall $\mathrm{MN}$ population. Thus, in order to limit the cell size analysis of Dlk1 expressing MNs towards alpha fast and slow MNs another experiment was performed using the alpha $\mathrm{MN}$ marker to discrimnate between the different $\mathrm{MN}$ classes. 


\section{Results}

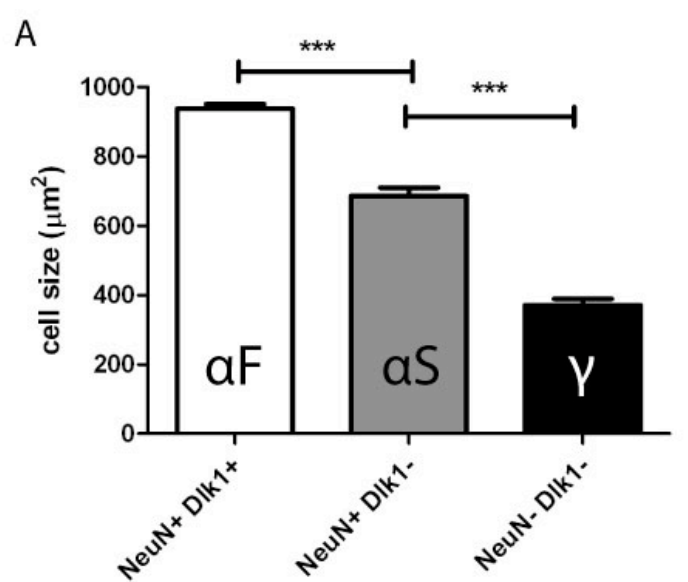

B

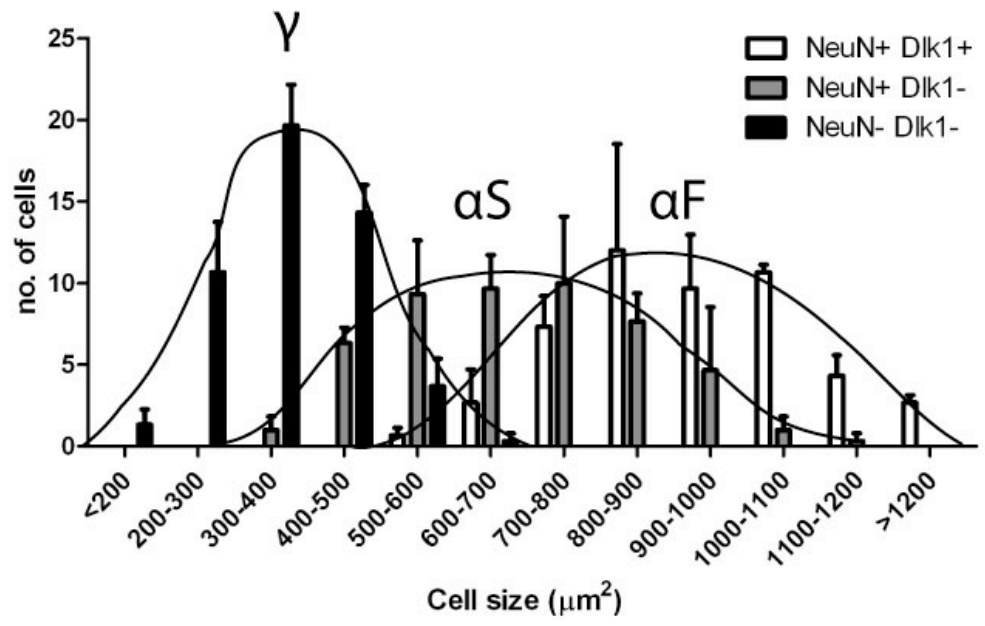

Fig. 5.16 Cell size quantification of different motor neuron populations at P4 Average cell size (A) and cell size distribution (B) of putative fast alpha MNs $(\mathrm{NeuN}+, \mathrm{Dlk} 1+)$, putative slow alpha MNs $(\mathrm{NeuN}+\mathrm{Dlk} 1+)$ and gamma motor neurons (NeuN-) at P4. Fast alpha MN $(\alpha \mathrm{F})$, slow alpha $\mathrm{MN}(\alpha \mathrm{S})$, gamma $\mathrm{MN}(\gamma)$.

The analysis of Dlk1 positive and Dlk1 negative alpha MNs exhibited a clear difference in cell size between the two cell populations based on average cell size as well as cell size distribution. Throughout the analysis no Dlk1 positive, but NeuN negative cells could be observed. Thus, the expression of Dlk1 is indicated to be restricted towards alpha MNs. The cell size of the average Dlk1 positive MN was $938.05 \mu \mathrm{m}^{2}\left(\mathrm{SD}+/-13.19 \mu \mathrm{m}^{2}\right)$ while other alpha MNs had an average soma size of $686.14 \mu \mathrm{m}^{2}$ (SD $+/-24.47 \mu \mathrm{m}^{2}$ ). Thus, Dlk1 expression is selectively associated with large soma size putative fast alpha MNs. 


\section{Results}

\subsubsection{Cell soma size distribution of Dlk1 expressing cells in different motor pools}

In order to verify, whereas these results that suggest Dlk1 expression correlated with putative fast motor neurons the cell soma size of motor neurons in different motor pools was correlated with the Dlk1 expression. This was furthermore necessary to exclude motor pool specific differences in MN cell soma sizes. At P4 four different motor pools were analyzed after retrograde tracing (Soleus, Rectus femoris, Tibialis cranialis and Triceps brachii). As Dlk1 is not expressed in motor neurons at P20 and onwards a cell size correlation was not performed at that stage. Retrogradely traced motor pools from three bilaterally injected animals were quantified $(n=3)$. Form each animal 100 randomly selected retrogradely labeled cells were examined.

A Soleus

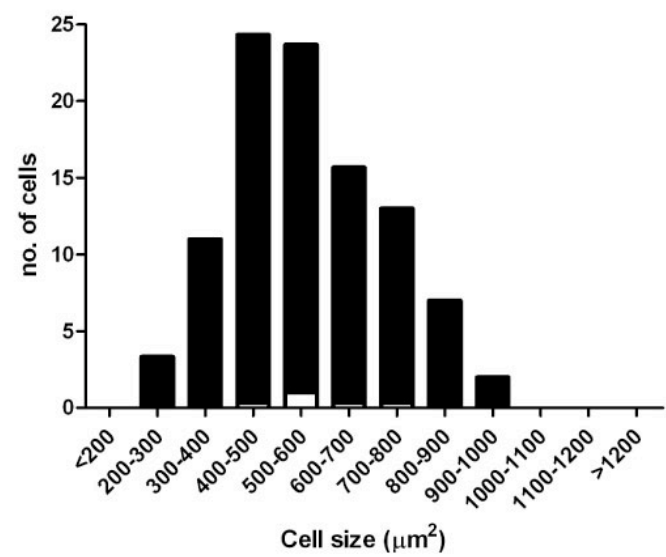

C Tibialis cranialis

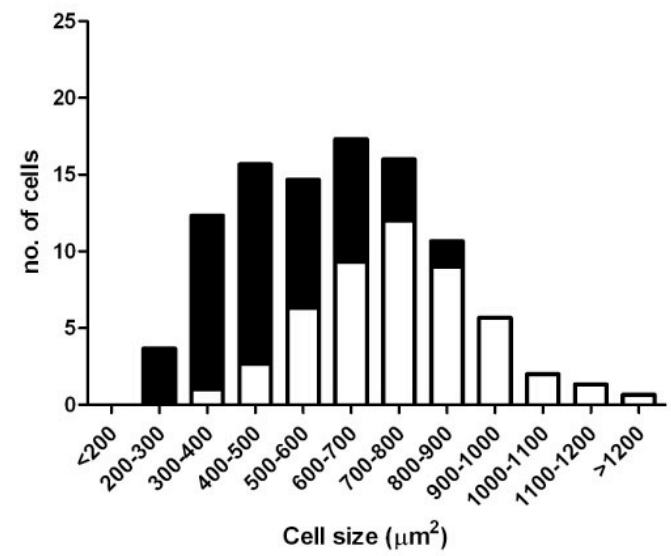

B Rectus femoris

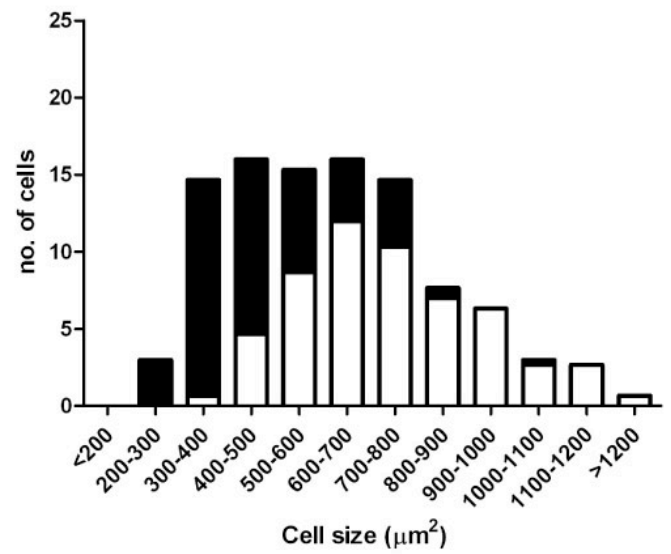

D Triceps brachii

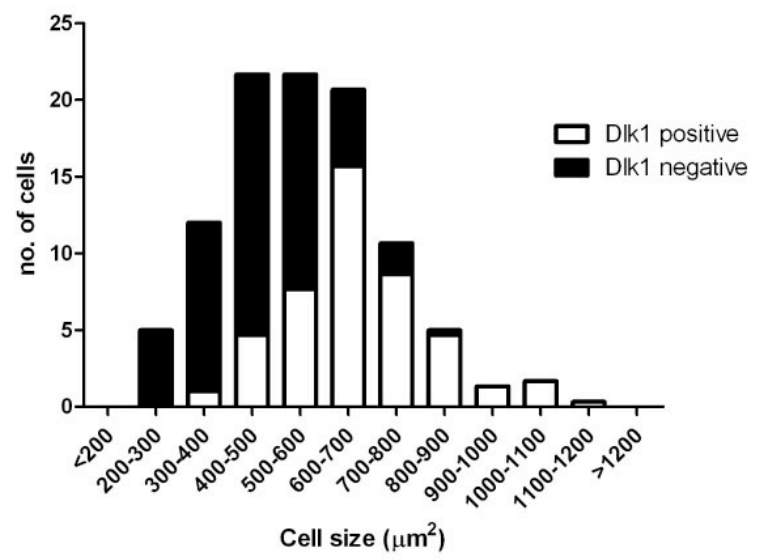

Fig. 5.17 Size distribution of Dlk1 positive motor neurons in different motor pools at P4 Cell soma size distribution of Dlk1 positive and negative cells in retrogradly traced Soleus (A), Rectus femoris (B), Tibialis cranialis (C) and Triceps brachii (D) motor pools in P4 mice. 


\section{Results}

Expression of Dlk1 was predominantly detectable within large soma size motor neurons in all examined motor pools at P4. The absence of Dlk1 in small size MNs throughout the analyzed motor pools makes motor pool specific variances for FMNT specific expression of Dlk1 to appear unlikely. Thus, it could be stated that the protein is a marker for fast FMNTs throughout the spinal cord MNs. Within the individual pools Dlk1 positive and negative cells could be distinguished based on the average cell soma size. However, it is not possible to state a clear cutoff value for the populations. A smaller average cell soma size of the Dlk1 negative cells in the Rectus femoris motor pool was found compared to the Soleus motor pool. This concurs with the anticipation that there is approximately the same number of gamma MNs within both pools, while there is a considerably higher number of alpha slow MNs present in the soleus motor pool. In general the MNs of the Triceps brachii motor pool appeared to be smaller in comparison to these from other mainly fast motor pools. This could indicate a general size difference in between brachial LMC and lumbar LMC MNs, however it is necessary to compare these results towards other brachial LMC motor pools. 


\section{Results}
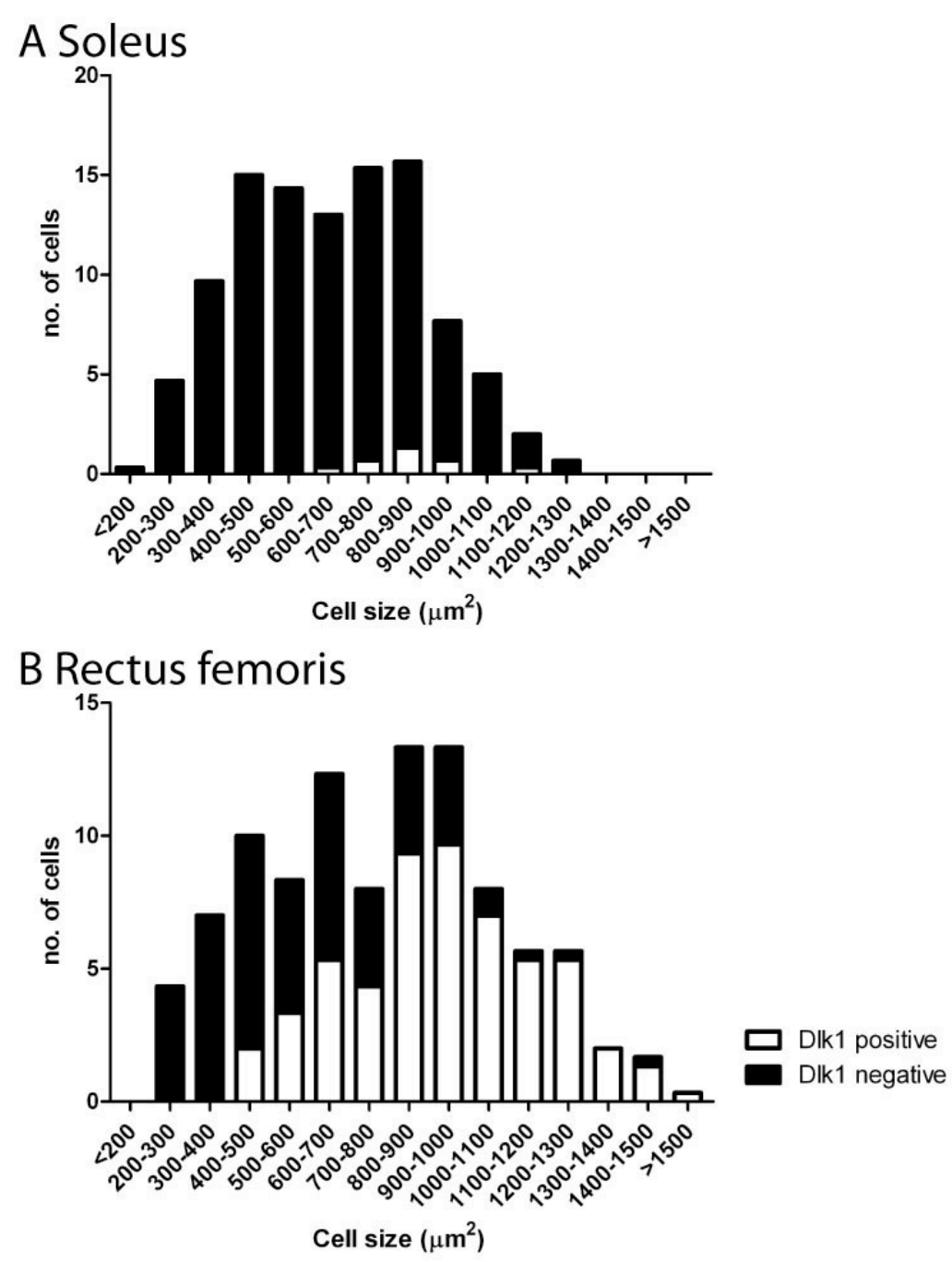

Fig. 5.18 Size distribution of Dlk1 positive motor neurons in the Soleus and Rectus femoris motor pool at P10 Cell soma size distribution of Dlk1 positive and negative cells in retrogradely traced Soleus (A) and Rectus femoris (B) motor pools in P10 mice.

The results at the P10 stage verified the results obtained at the P4 stage. Dlk1 is still expressed in the large soma size fraction of the retrogradely labeled MNs.

Together, these data show that Dlk1 is expressed in a discrete subset of large soma size alpha MNs. Also its expression positively correlates with the predicted fast MN contents of different motor pools. Based on al criteria it is therefore concluded that Dlk1 is selectively expressed in fast MNs.

\subsection{Dlk1 expression during development}

Our initial results and the following qualitative and quantitative examinations were carried out at early postnatal stages P4 and P10 during which FMNTs are likely to be specified. It was next asked how the expression of Dlk1 is regulated during embryonic 


\section{Results}

development as well as in adult animals. The examination of the embryonic expression would allow getting further insights into the process of FMNT formation. On the other hand, a marker for adult stage FMNTs would be useful to monitor the progression of motor neuron diseases in direct association with the different FMNTs.

\subsubsection{Dlk1 expression in the developing mouse embryo}

Immunohistochemical analysis of Dlk1 expression is difficult at embryonic stages, since the protein shows a strong membrane association at that time. This makes it challenging to pinpoint the signal to discrete cells. Therefore the expression analysis was performed by in-situ hybridization to detect the mRNA transcripts and thereby facilitating more precise statements regarding the position of the actual Dlk1 expressing cells. Embryos at four different stages were analyzed starting with E10.5 as motor neurons are born at E10 onwards. 


\section{Results}
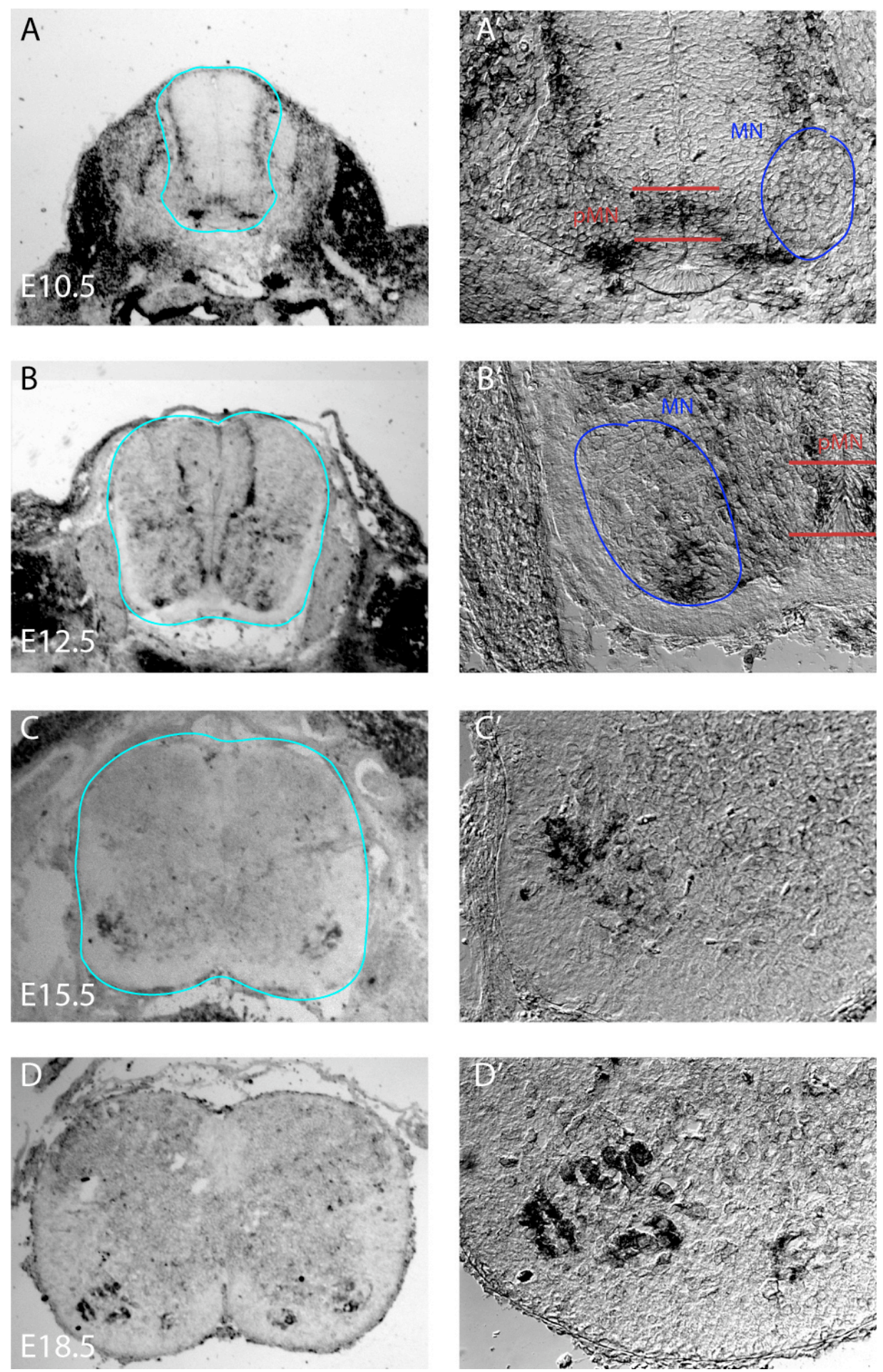

Fig. 5.19 In-situ analysis of Dlk1 expression in the embryonic mouse spinal cord at different stages D1k1 expression at E10.5, E12.5 E15.5 and E18.5 stage at 40x (A,B,C and D) and 200x (A',B',C' and D') magnification. The boundaries of the spinal cord are marked in cyan in the embryonic stages E10.5, E12.5 and E15.5. The position of the motor neuron progenitor $(\mathrm{pMN})$ domain and the postmitotic motor neurons $(\mathrm{MN})$ is indicated in $\mathrm{A}^{\prime}$ and $\mathrm{B}^{\prime}$. 


\section{Results}

Initial Dlk1 expression in mouse is detectable at embryonic day 10.5 by northern blot analysis in whole embryo RNA and in the developing vertebra at E13 by in-situ hybridization according to Smas \& Sul (1993).

At E10.5 the analysis in this work revealed presence of Dlk1 in the pMN a discrete region of the spinal cord containing the progenitor cells from which MNs are generated, putative V3 interneurons and in post-mitotic neurons of the mantle layer. However, Dlk1 was not detectable within postmitotic motor neurons at this stage. Besides the expression in the spinal cord Dlk1 is highly expressed in the developing myoderm. In E12.5 embryos, Dlk1 mRNA could be detected in a population of cells in the ventral horn whose position corresponds to that of postmitotic motor neurons. At the same time the pMN progenitors and a stripe adjacent to ventricular zone are expressing Dlk1. These cells have recently exited the cell cycle and are now migrating towards their final position. Thus, Dlk1 expression could hallmark an intermediate progenitor state of these cells. Noticeably the Dlk1 positive motor neurons seem to cluster, while there are other clusters of Dlk1 negative cells within the motor columns. In a later embryonic stage at E15.5 the expression of Dlk1 is extinguished in the pMN domain while expression within postmitotic MNs is retained. Furthermore, some single cells close to the midline seem to be Dlk1 positive, but they do not form a distinct domain. At E18.5 the Dlk1 expression pattern was already similar to the postnatal stages and resembled the "salt and pepper" pattern that suggests FMNT specific expression. The early expression of Dlk1 in a subset of postmitotic MNs from E12,5 onwards could therefore point to an unanticipated early specification of FMNTs.

\subsubsection{Correlation of embryonic Dlk1 expression to the motor neuron markers}

As already mentioned the membrane localization of the Dlk1 protein within the early embryonic stages makes it difficult to colocalize the expression with MN markers in immunohistological stainings. 


\section{Results}
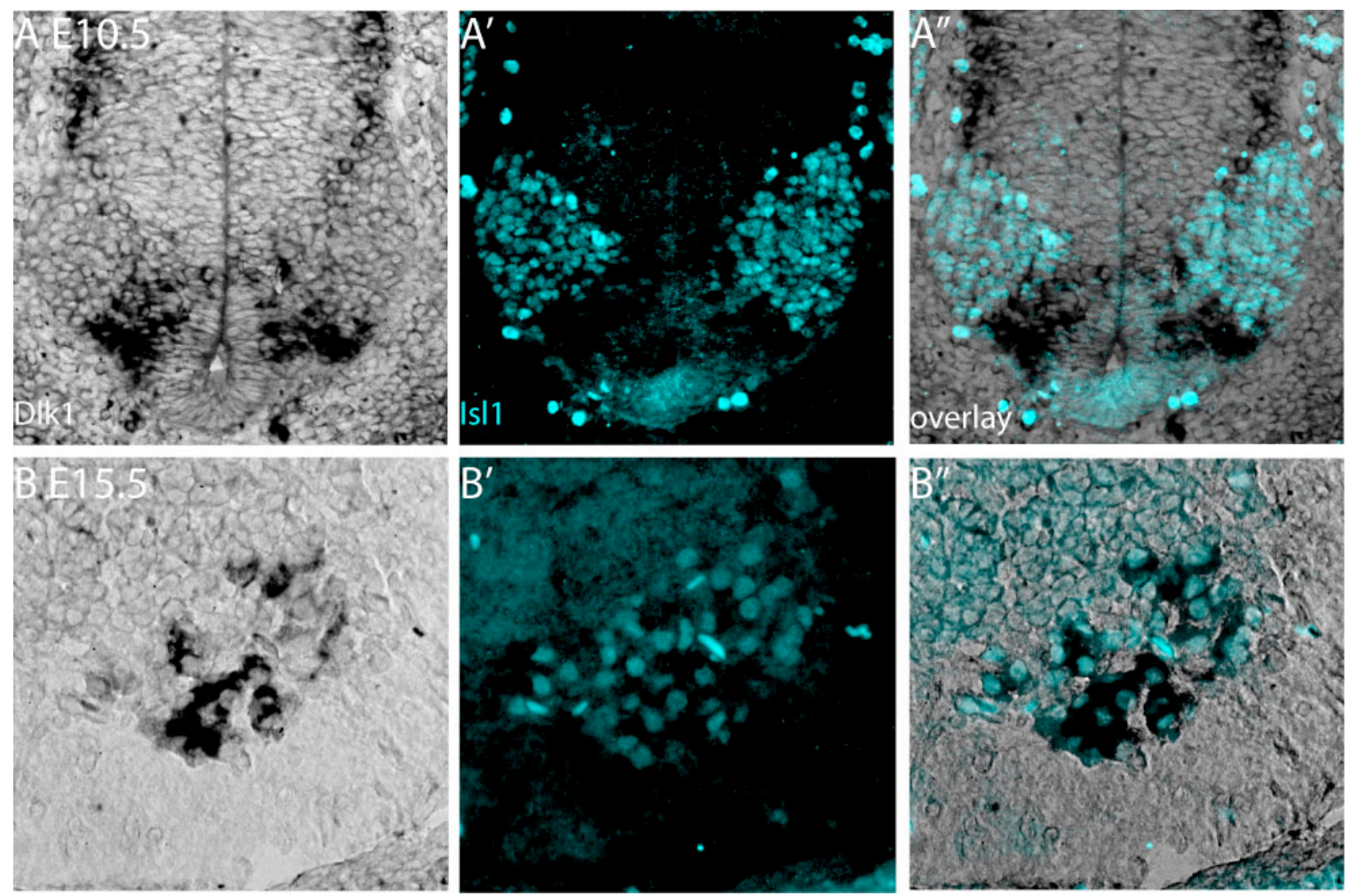

Fig. 5.20 In-situ hybridization for Dlk1 combined with anti-Isl1 antibody staining at E10.5 and E15.5 Dlk1 expression detected by Dlk1 antisense riboprobe (A, B). anti-Isl1 immunostaining (A', B'). Overlay picture (A', B' ').

Hence, Dlk1 in-situ hybridization and subsequent immunostaining for Islet 1 (Isl1) was combined to verify the early embryonic expression pattern in MNs. As it was already suggested by the initial analysis, Dlk1 transcripts could not be detected within Isl $1{ }^{+}$postmitotic motor neurons at E10.5. It seemed to be excluded from postmitotic motor neurons at that stage. But at the later embryonic stage E15.5 Dlk1 transcripts are present within a subpopulation of motor neurons while they are other MNs that were negative for Dlk1 RNA. Like the previous analysis the in-situ pattern showed clusters of Dlk1 positive cells while on the other side Dlk1 negative cells clustered, which may indicate cell non-autonomous signaling in between these MNs. 


\section{Results}

\subsubsection{Expression of Dlk1 in different postnatal stages}

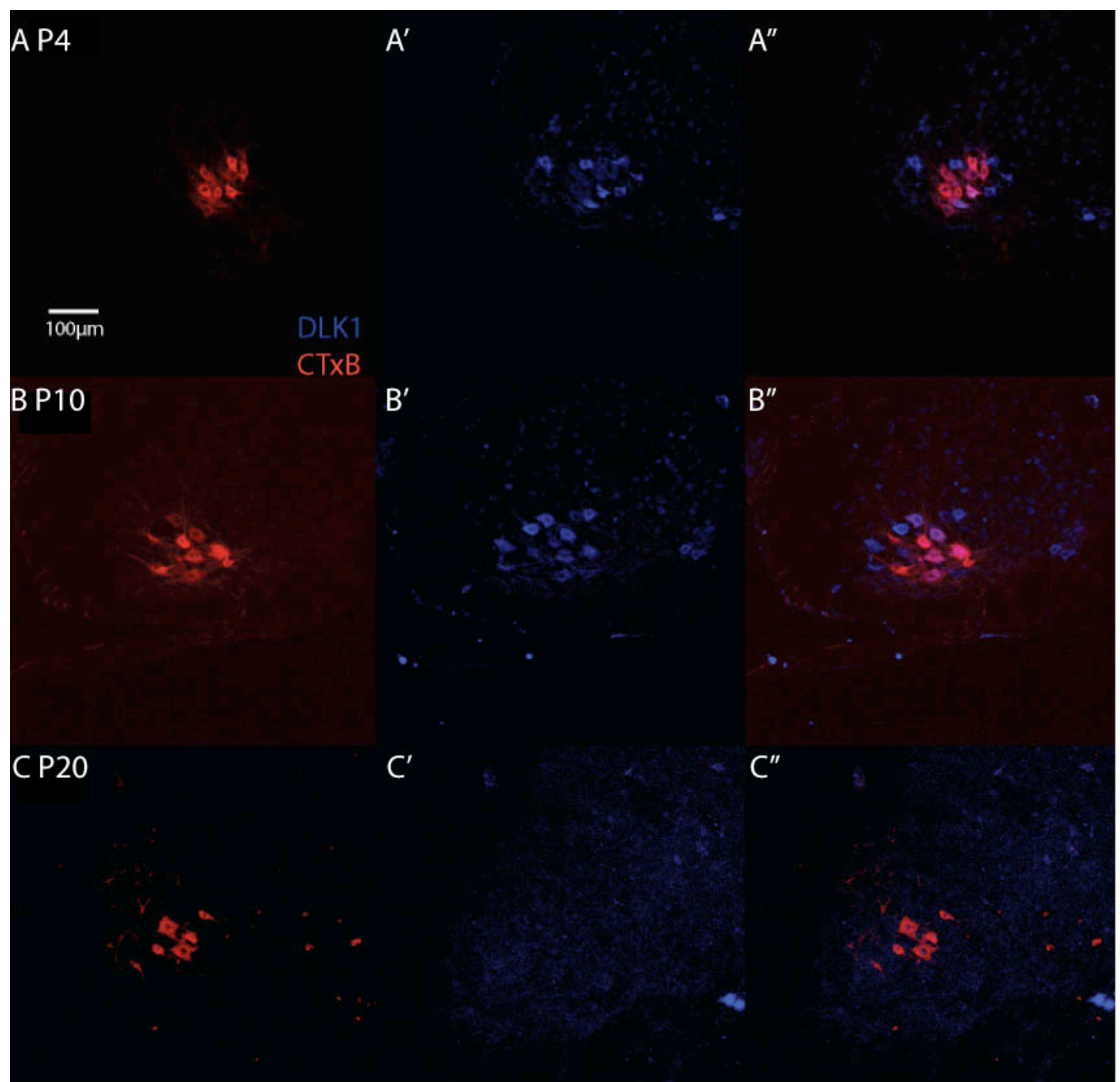

Fig. 5.21 Dlk1 immunostaining at mouse spinals cord from different postnatal stages Dlk1 expression was not detectable any more in motor neurons at P20 and at adult stages.

Dlk1 is not expressed in motor neurons any more in developmental stages from P20 onwards or in adults. But Dlk1 was still found to be present within a yet not defined population of cells within the spinal cord according to immunohistochemical analyis and in-situ hybridisation. Taken together these findings indicate that Dlk1 is present in MNs exclusively during the development and the expression is reduced as soon as the cells are mature. Unfortunately this result denies the use the gene as a marker in later stages in order to monitor specific FMNTs in neuromuscular diseases. 


\section{Results}

\subsection{Analysis of Dlk1 knockout mice}

\subsubsection{Genotyping and verification of the Dlk1 knockout mice}

To test whether Dlk1 plays a role in FMNT specification it was decided to study the genes function using Dlkl knockout mice. Therefore the mouse line described by Raghunandan et al. was obtained (Raghanundan et al. 2008). To check for the presence of remaining expression of truncated mRNA fragments that might cause a dominant negative effect within in $D l k 1^{-/}$animals an in-situ hybridization analysis was performed.
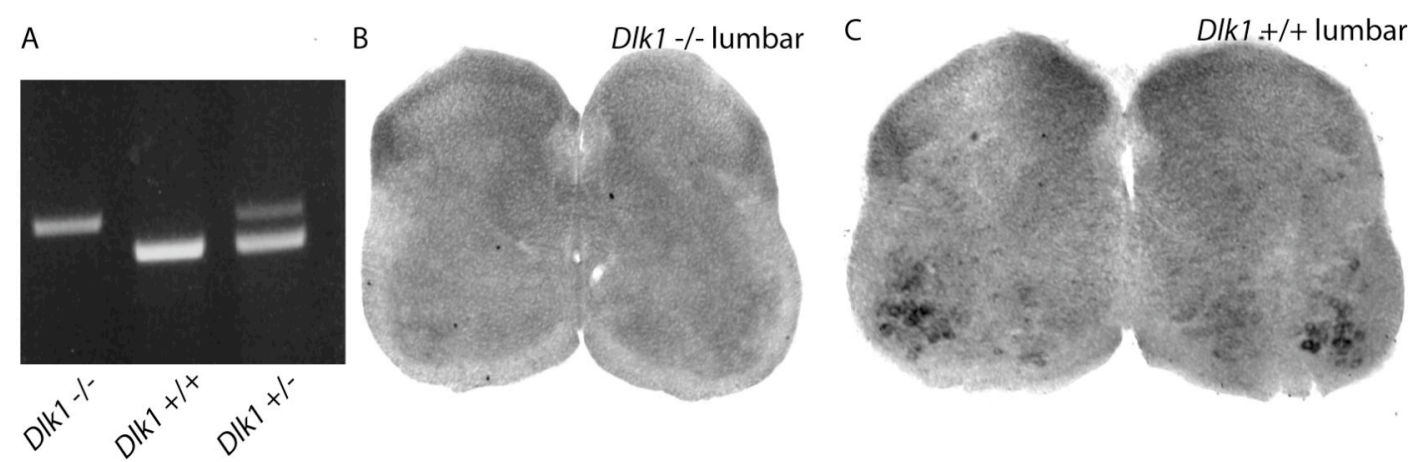

Fig. 5.22 Genotyping of Dlk1 knockout mice and verification of Dlk1 loss Agarose gel analysis of Dlkl genotypes (A). In-situ hybridization of the lumbar spinal cord of Dlkl knockout (B) and wild type mouse at P4 (C).

Genotyping of Dlkl knockout mice was carried out via PCR on DNA extracted from tail biopsies. The gel electrophoresis pattern was the same that could be observed in the original publication by Raghunandan et al. with a single band at $414 \mathrm{bp}$ for the Dlk1 wild-type animals, a single band at 495bp for homozygous knockout mice and both bands in case of heterozygous animals. As $D l k l^{-/-}$animals are viable and do not show obvious defects in fertility, they were kept homozygous. Dlk1 expression was detectable neither via in-situ hybridization nor via immunohistological stainings with the Dlk1 antibody. Thus, as the mouse strain lacks both Dlk1 protein and RNA effects caused by a hypomorphic allele could be excluded.

\subsubsection{Characterization of Dlk1 knockout animals}

The Dlk1 knockout animals did not show obvious defects besides higher body weight due to increased body fat. The defects in eye or rip cage development depicted by Moon and colleagues have not been observed so far (Moon et al. 2002). Dlkl ${ }^{-1}$ animals were born in the expected Medelian ration. Also, no increased early postnatal 


\section{Results}

mortality or reduced birth weight was found in $D l k 1^{-/-}$animals compared to their wild type littermates. These differences might be caused by differing genetic backgrounds of the mouse lines or defects carried over from the embryonic stem cells used to generate these mice.

\subsubsection{The clasping phenotype in Dlk1 knockout mice}

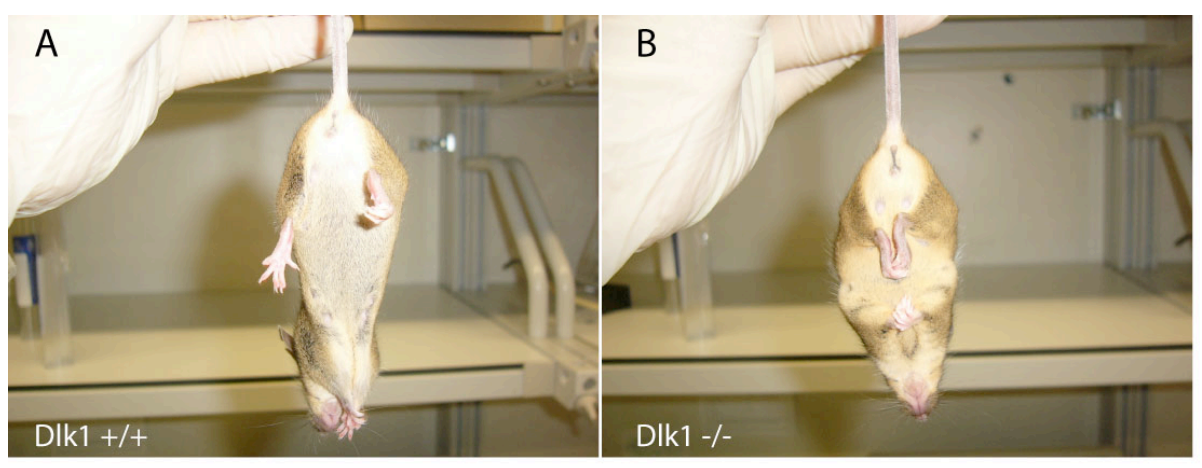

C

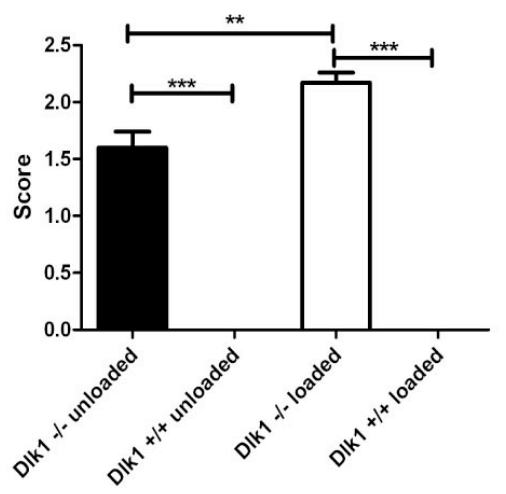

D

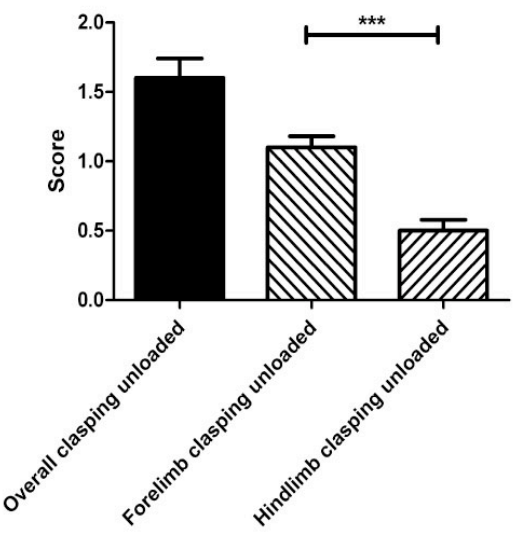

E

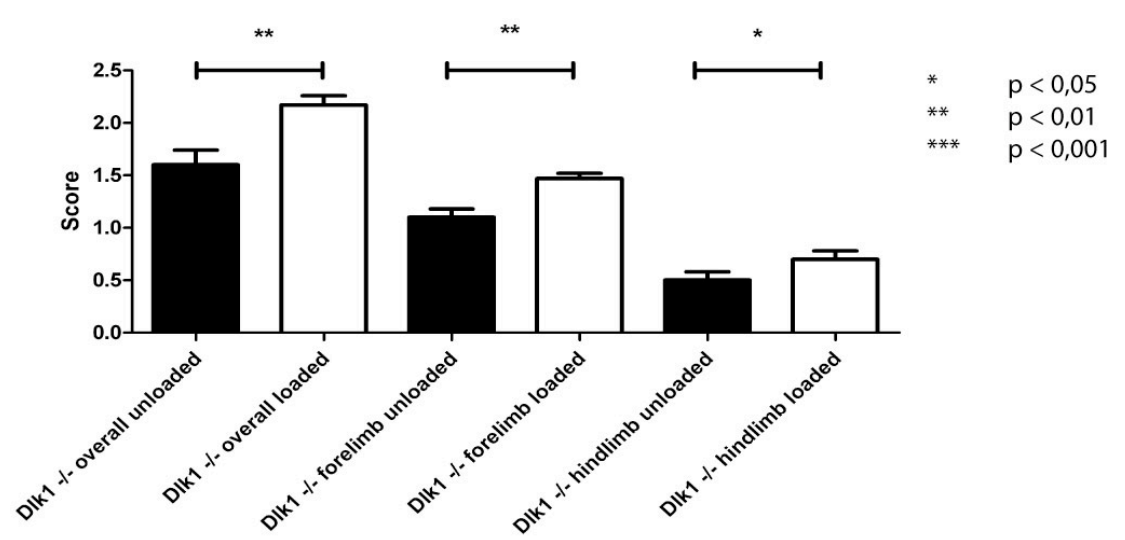

Fig. 5.23 The clasping phenotype in Dlk1 knockout mice Dlkl wild type animal in comparison to a knockout mouse showing strong a clasping behavior (A, B). Clasping behavior in Dlkl knockout and wild type animals before (unloaded) and after (loaded) running exercise (C). Clasping score for fore and hindlimb before running exercise (D). A summary of clasping scores before and after running exercise (E). 


\section{Results}

Dlk1 knockout animals exhibited a neurological impairment that resulted in a pathological fore and hind limb clasping behavior when the animals were subjected to tail suspension (Fig. 5.23 A \& B). This phenotype was typically observed several seconds after suspension. To examine this phenotype, the mice response towards tail suspension was monitored and scored according to Methods 4.1.3.3. None of the wild type animals showed clasping in any of the trials (Fig. $5.23 \mathrm{C}$ ). The phenotype affected the forelimbs significantly stronger than the hind limbs (Fig. 5.23 D), which differs from other transgenic mouse models for neuromuscular diseases where it is more prominent in the hindlimbs (Mangiarini et al. 1996, Guy et al. 2001, Hansson et al. 2003, Bruestle et al. 2009). In order to test how increased neuromuscular workload is affecting the phenotype the animals were tested for clasping before and directly after being subjected to a short high intensity running exercise. After the running exercise the average clasping score increased by app. 35\% to 2.166 points while wild type animals still did not show clasping (Fig. $5.23 \mathrm{C}$ ). These results indicate that neuromuscular stress enhances the pathological clasping phenotype possibly due to energy depletion or oxidative stress in the muscles or the associated MNs of Dlk1 knockout mice. 


\section{Results}

\subsubsection{Running endurance test}

The specification of FMNTs is directly linked to the fine tuned regulation of skeletal muscle outputs. Thus, to generally test neuromuscular performance of $D l k l^{-/}$animals, they were subjected to two forced treadmill running endurance paradigms. Running endurance assays are generally applied to test the integrity of the neuromuscular systems and voluntary running distance was used as a diagnostic marker for the disease progression in mouse models of neuromuscular diseases. Two different protocols were applied in this assay in order to test the performance of different motor units. The medium intensity protocol tested the ability to run at a gradually increasing speed on a slight slope of $10^{\circ}$ while the high intensity protocol used the same running speeds but in combination with a $20^{\circ}$ incline.
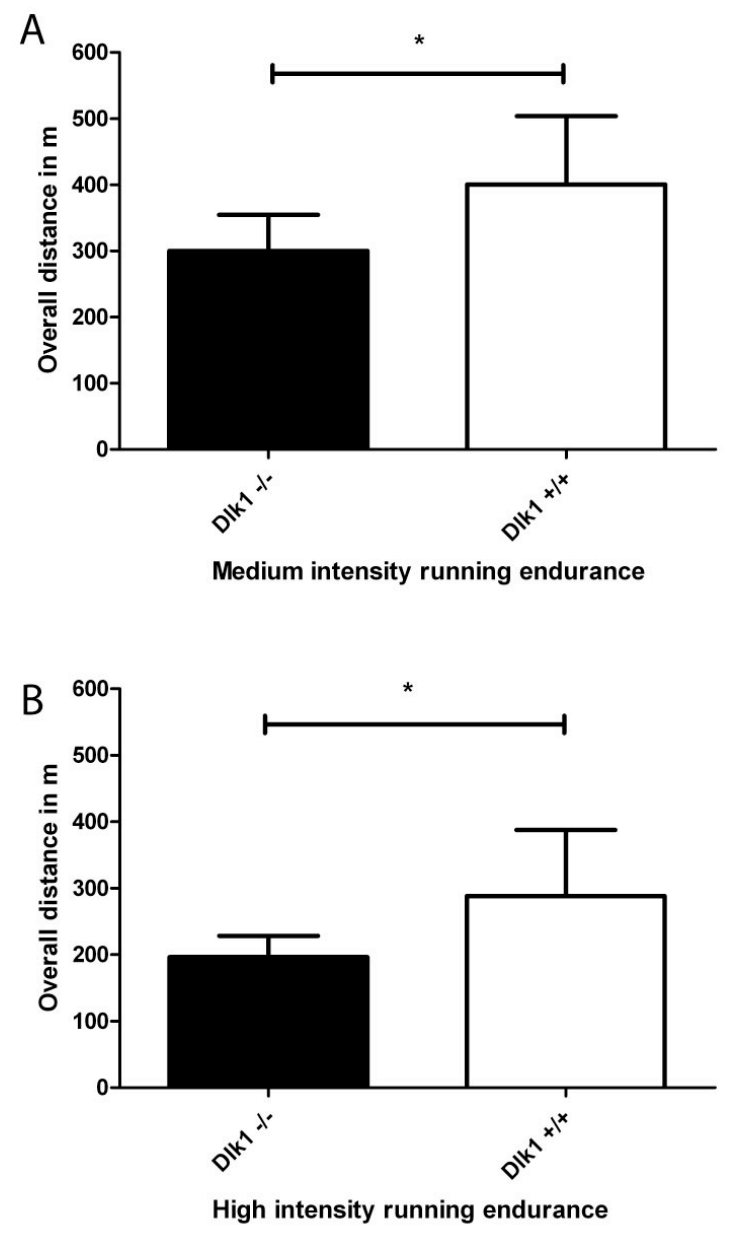

Fig. 5.24 Running endurance of Dlk1 knock out and wild-type mice Dlk1 knockout and wildtype animals were subjected to a treadmill running protocol at $10^{\circ}$ (A) and $20^{\circ}$ (B) incline. The test was performed with six Dlkl wild-type control mice and ten $D l k 1^{-/-}$experimental animals. 


\section{Results}

In this assays the running endurance of $D l k l^{-/-}$mice was reduced compared to wildtype animals in the medium and high intensity running protocol. Although there were high individual differences observed between the experimental animals the results were significant $(\mathrm{p}<0.05)$ in this assay. In the medium intensity running paradigm the Dlkl knockout mice traveled a distance of $300.1 \mathrm{~m}$ (SD +/-54.31 m) while the wild type controls reached a distance of $400.5 \mathrm{~m}(\mathrm{SD}+/-103.15 \mathrm{~m})$. These results were verified by the high intensity running test in which the Dlkl knockout mice traveled a distance $196.6 \mathrm{~m}(\mathrm{SD}+/-31.8 \mathrm{~m})$ compared to $288.1 \mathrm{~m}(\mathrm{SD}+/-99.5 \mathrm{~m})$ for the wildtype controls. However, due to the systemic loss of the protein to this point it could not be determined if the observed phenotype was caused by a neuronal impairment, a muscle defect or the higher body weight of the $D l k 1^{-/-}$animals.

\subsubsection{Grip strength assay}

Forelimb grip strength assays allow testing the neuromuscular performance of rodents. This is based on the grasping reflex that makes the animals holding on to a handlebar upon being pulled backwards. Grip strength is also routinely used as an indicator for neuromuscular disease progression (Henriques et al. 2010). The grip strength of ten male Dlk1-/- and six male Dlk1 +/+ control mice was examined at three subsequent days. Each animal was tested five times per day and the highest value was taken.

The Dlkl knockout animals revealed significantly lower absolute and relative grip strength compared to wild type mice. The absolute grip strength decreased from $164 \mathrm{~g}$ (SD +/-6.84 g) in wild-type animals to $138 \mathrm{~g}$ ( $\mathrm{SD}+/-4.64 \mathrm{~g}$ ) in knockout mice. A similar result was obtained for the relative grip strength, which was reduced from $6.35 \mathrm{~g} / \mathrm{g}$ body weight ( $\mathrm{SD}+/-0.32 \mathrm{~g}$ ) to $5.14 \mathrm{~g} / \mathrm{g}$ body weight (SD $+/-0.47 \mathrm{~g}$ ). The individual variances observed in this experiment were lower than that observed in running endurance test. However, the measured force values slightyly increased over the three experimental days suggesting a possible familiarization of the animals towards the experimental setup. However, as stated for the impairment in running endurance observed in these animals also this phenotype might be caused due to a systemic effect reducing general neuromuscular performance. Thus, to obtain a more direct measurement of the effects of Dlk1 towards neuromuscular performance, it will 


\section{Results}

be necessary to repeat the experiments with animals selectively deficient of Dlk1 in MNs as soon as they become available.
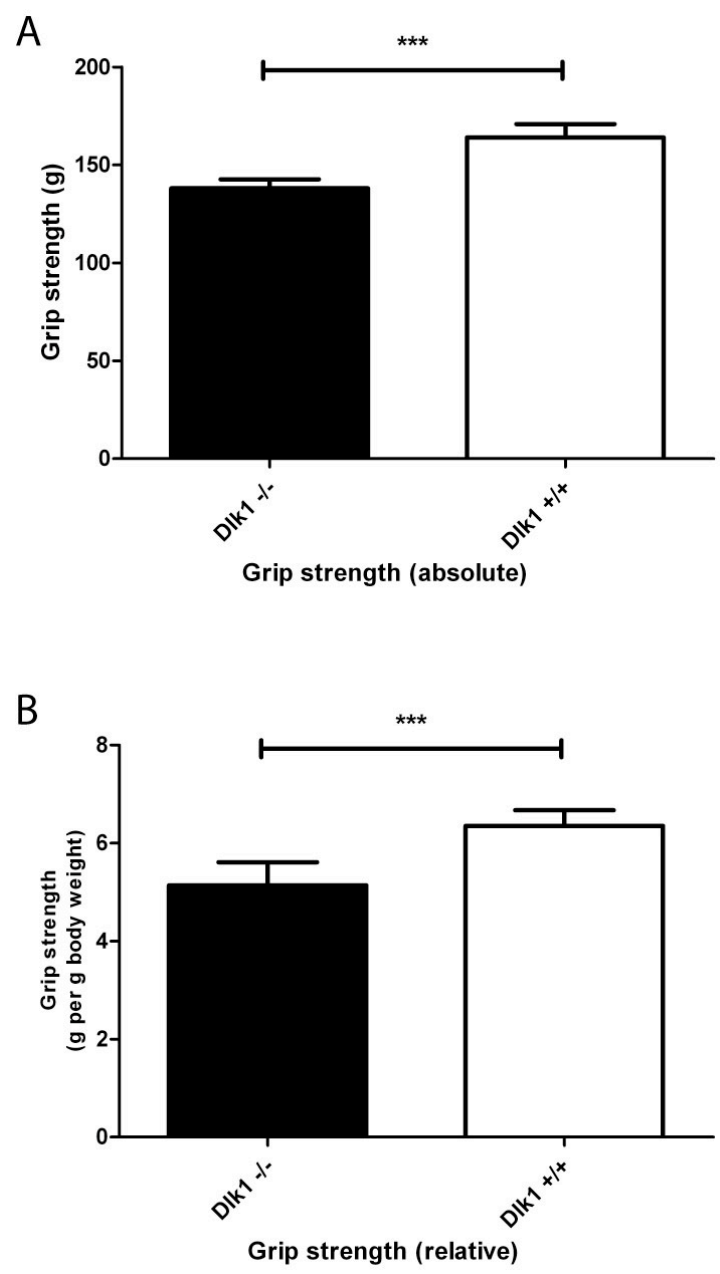

Fig. 5.25 Grip strength measurement of Dlk1 knockout and wild-type mice Ten four month old knockout and six age matched wild-type control animals were tested. Error bars are indicating standard deviation. The absolute grip strength (A) as well as the grip strength normalized to body weight (B) was determined.

\subsubsection{Effects of Dlk1 loss in motor neuron gene signature}

Due to its similarity to the Delta protein family Notch ligands which regulate cell fate it was next addressed whether loss of Dlk1 overall affect the specification of MNs. To address this, a microarray expression analysis of FMNTs in control and knockout animals was performed. The aim was to detect potential molecular targets of Dlk1 function and to obtain initial mechanistic insights into the Dlk1 pathway. The analysis of the Rectus femoris innervating MNs has proven to be technically difficult due to the relatively low number of cells labeled. Thus, for analysis of the fast MNs in Dlk1 


\section{Results}

knockout mice, the motor neurons innervating the quadriceps muscles (Rectus femoris, Vastus lateralis, Vastus medialis and Vastus intermedius), were retrogradly traced. Since the quadriceps muscles predominantly consist of fast muscle fibers. In parallel the Soleus muscle was injected to trace its associated predominantly slow MNs. Subsequently the MNs were extracted by fluorescence-guided microdissection and the transcriptomic profile of the cells was analyzed by microarray hybridization.

\subsubsection{Gene expression analysis of Dlk1-/- motor neurons}

The expression data gained from the microarray experiment was analyzed and the genes that have shown to be significantly differentially regulated were selected. A threshold value of 100 relative units was set in order to exclude genes that are unlikely to be expressed in MNs. The genes that have shown to be expressed at least 1.7 fold differentially in between Dlk1 wild type and knockout MNs in Quadriceps motor pools are displayed in the subsequent figures. 


\section{Results}

Genes upregulted in Dlk1 wild-type Quadriceps MNs

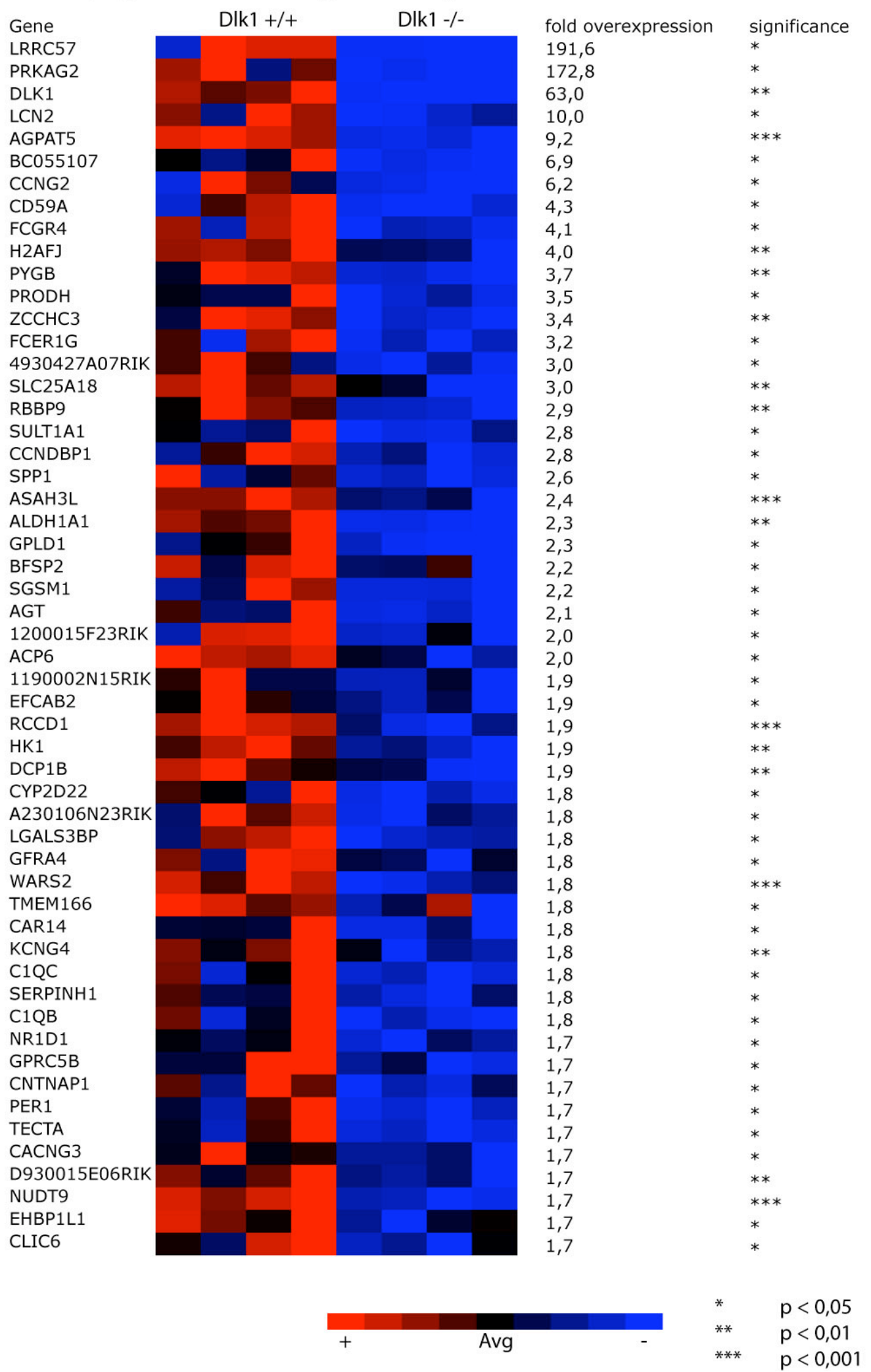

Fig. 5.26 Heatmap of the genes upregulated in Dlk1 wild-type Quadriceps MNs genes The 54 genes were found to be upregulated in the $D l k l$ wild type mice at least 1.7 fold. 


\section{Results}

\section{Genes upregulted in Dlk1 knockout Quadriceps MNs}

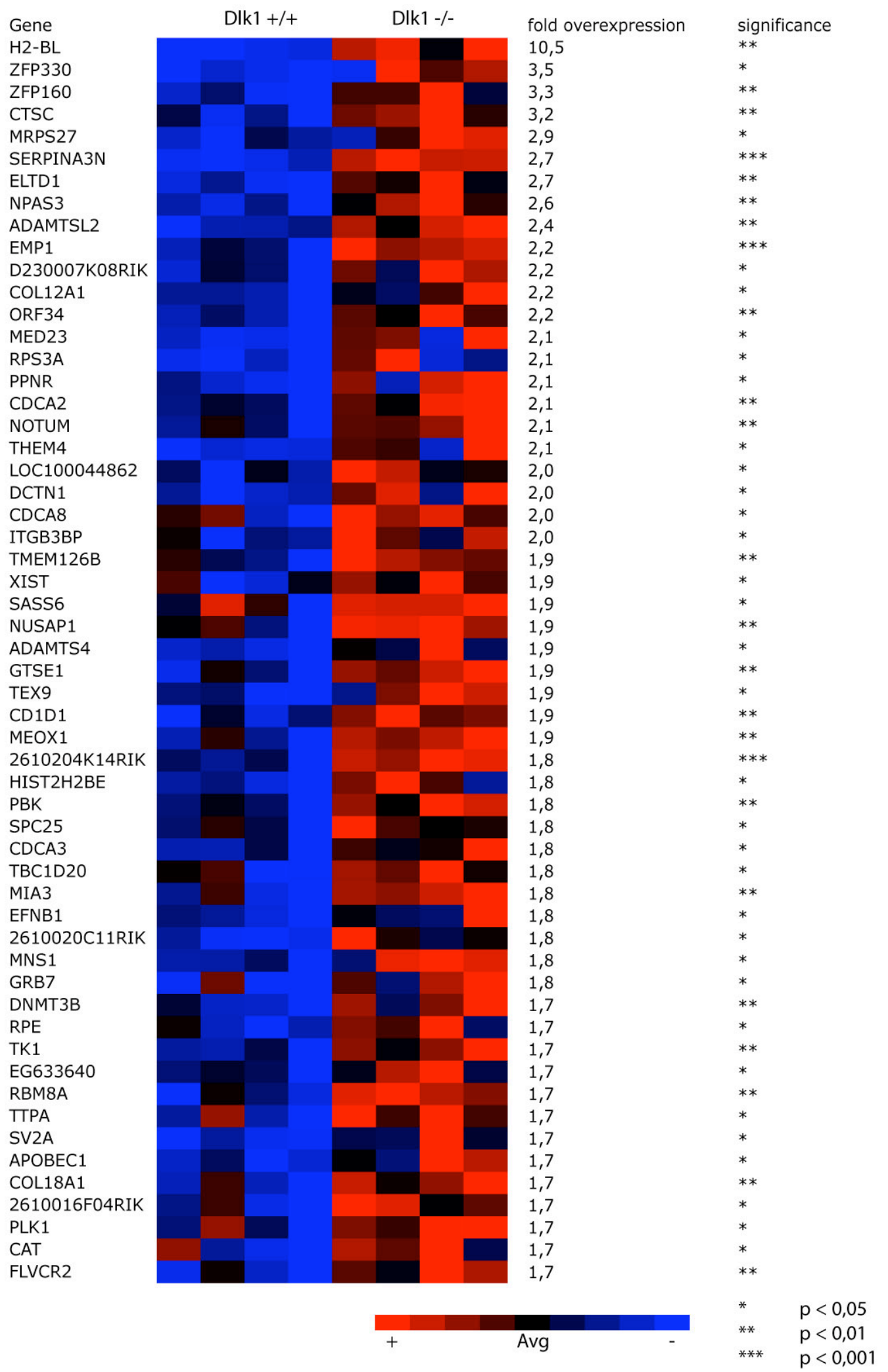

Fig. 5.27 Heatmap of the genes upregulated in Dlk1 knockout Quadriceps MNs 55 genes were upregulated at least 1.7 fold in the knockout MNs. 


\section{Results}

Analysis of the Quadriceps innervating MNs of $D l k I^{+/+}$and $D l k I^{-/-}$mice has yielded a large number of genes putatively linked towards Dlk1 function. However, as Dlk1 is expressed in a broad range of other tissues and cell types besides fast MNs, it has to be elucidated in which way systemic effects contribute to these results. Thus, the initial microarray experiment was repeated for the Soleus innervating MNs in $D l k l^{+/+}$ and $D l k l^{-/-}$animals.
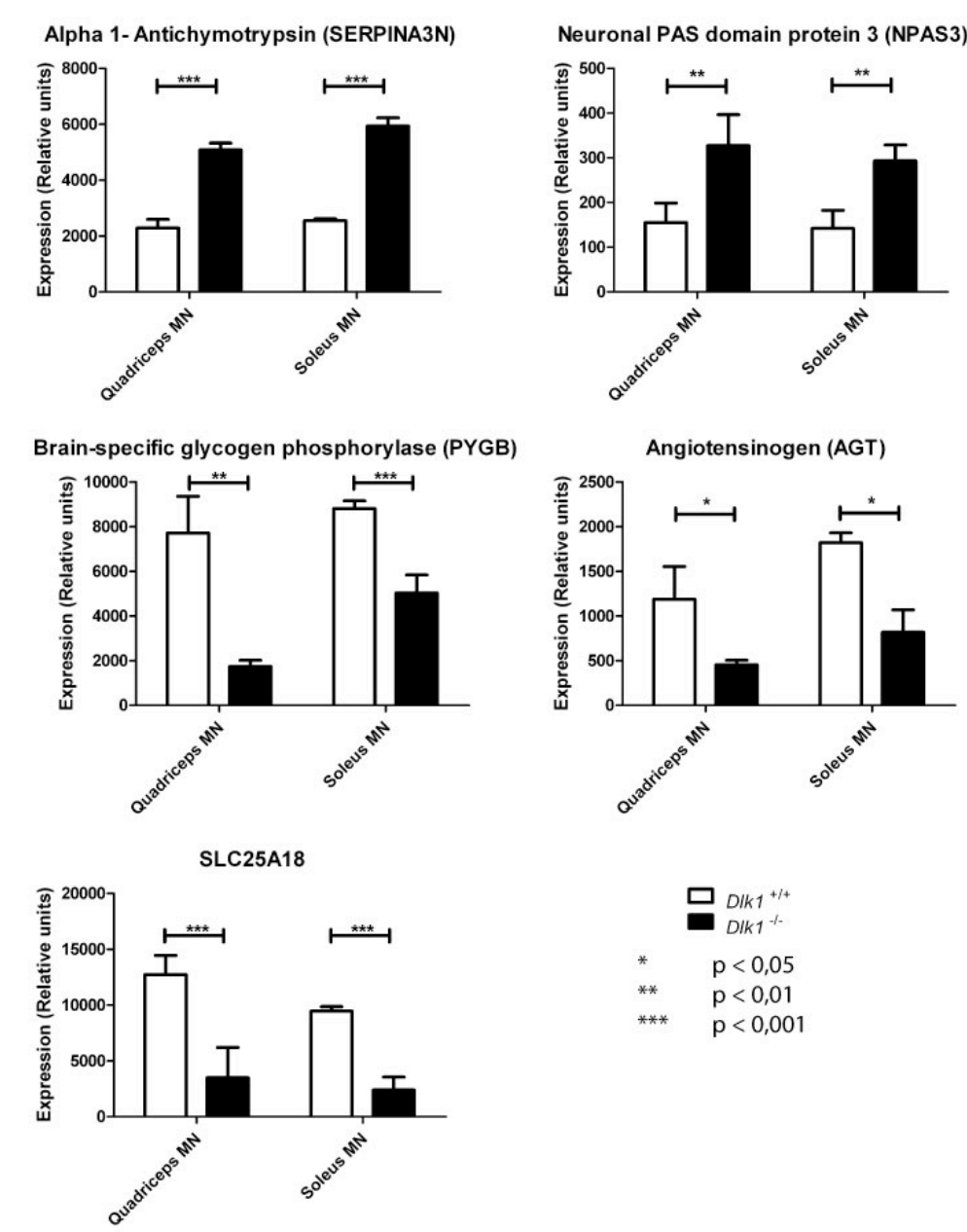

Fig. 5.28 Gene expression changes putatively linked to a systemic loss of Dlk1 function Loss of Dlk1 expression causes gene expression changes in fast as well as in slow MN enriched pools. Thus, these genes might be caused by a systemic effect of Dlk1 deficiency.

Loss of Dlk1 function has affected the expression of several genes in a FMNT independent manner (Fig. 5.28). Among these genes are SERPINA3N, NPAS3 and PYGB. However, it remains to be elucidated if exogenous effects of Dlk1 loss in peripheral tissues cause these gene expression changes, or occur in response to the lack of cell non-autonomous Dlk1 signaling from nearby fast MNs. 


\section{Results}
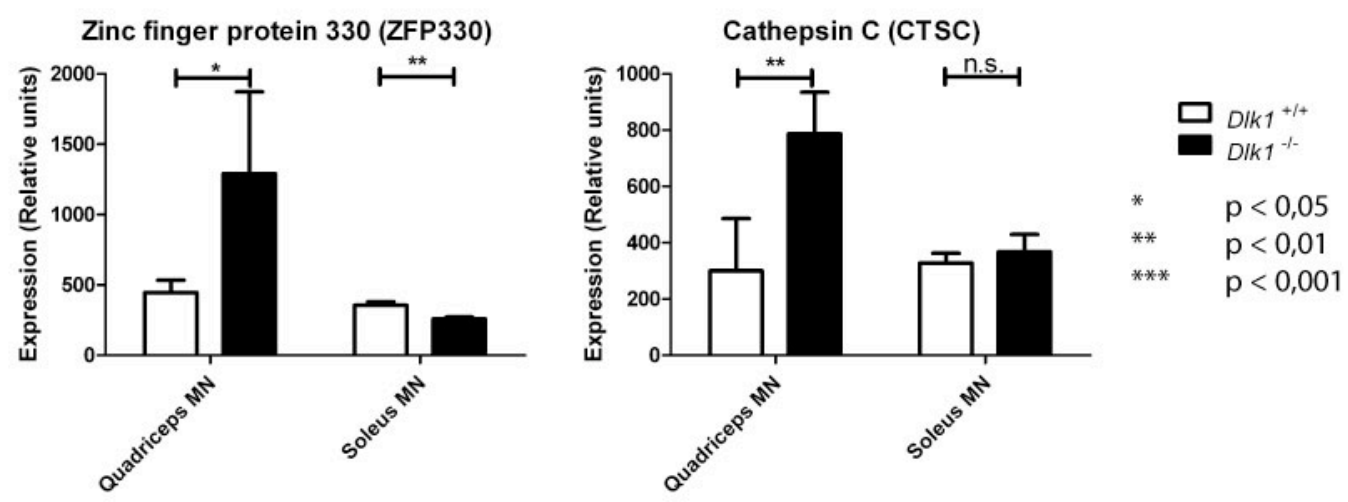

EGF-TM7-latrophilin-related protein (ELTD1)

1-acylglycerol-3-phosphate O-acyltransferase 5 (AGPAT5)
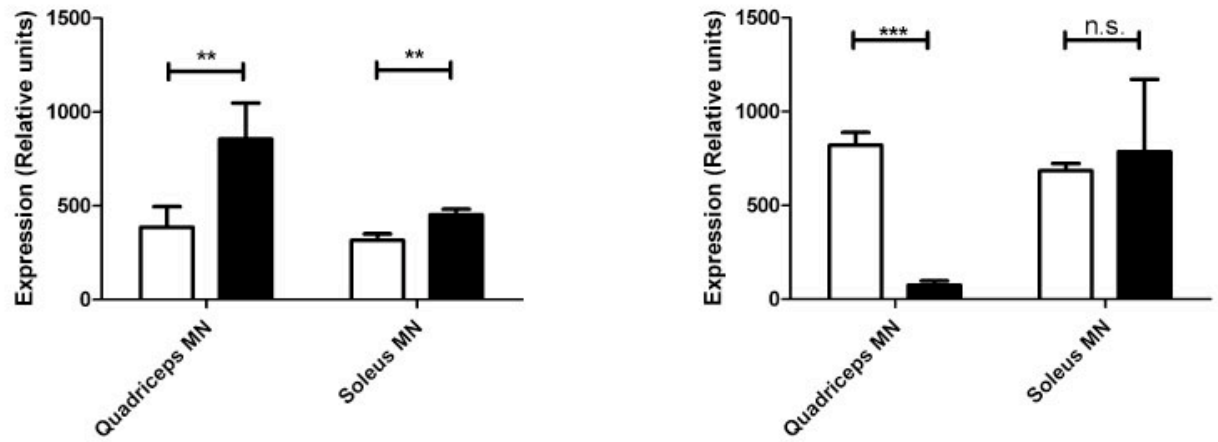

Fig. 5.29 Fast FMNT specific expression changes in $\mathbf{D l k} \boldsymbol{I}^{-/-}$mice Comparison of a selection of genes that are influenced by Dlk1 in a cell autonomous manner.

However, besides the systemic effects of Dlk1 loss, several genes change their expression profile in a FMNT specific manner in Dlk1 knockout mice (Fig. 5.29). These genes seem to be influenced in a cell autonomous fashion by Dlk1.

Another surprising finding was made during analysis of the gene expression profiles. A distinct set of genes like e.g. LRRC57, PRKAG2 and CCNDBP1 revealed a completely differing expression pattern in between Dlkl wild type and knockout animals. The expression that was initially higher in the Quadriceps wild-type MNs has switched and is found to be increased in the Soleus knockout MNs (Fig. 5.30). These results are not consistent with a cell autonomous role of Dlk1, instead they suggest the Dlk1 protein to regulate gene expression in nearby cell populations either in a juxtacrine or paracrine way. 


\section{Results}

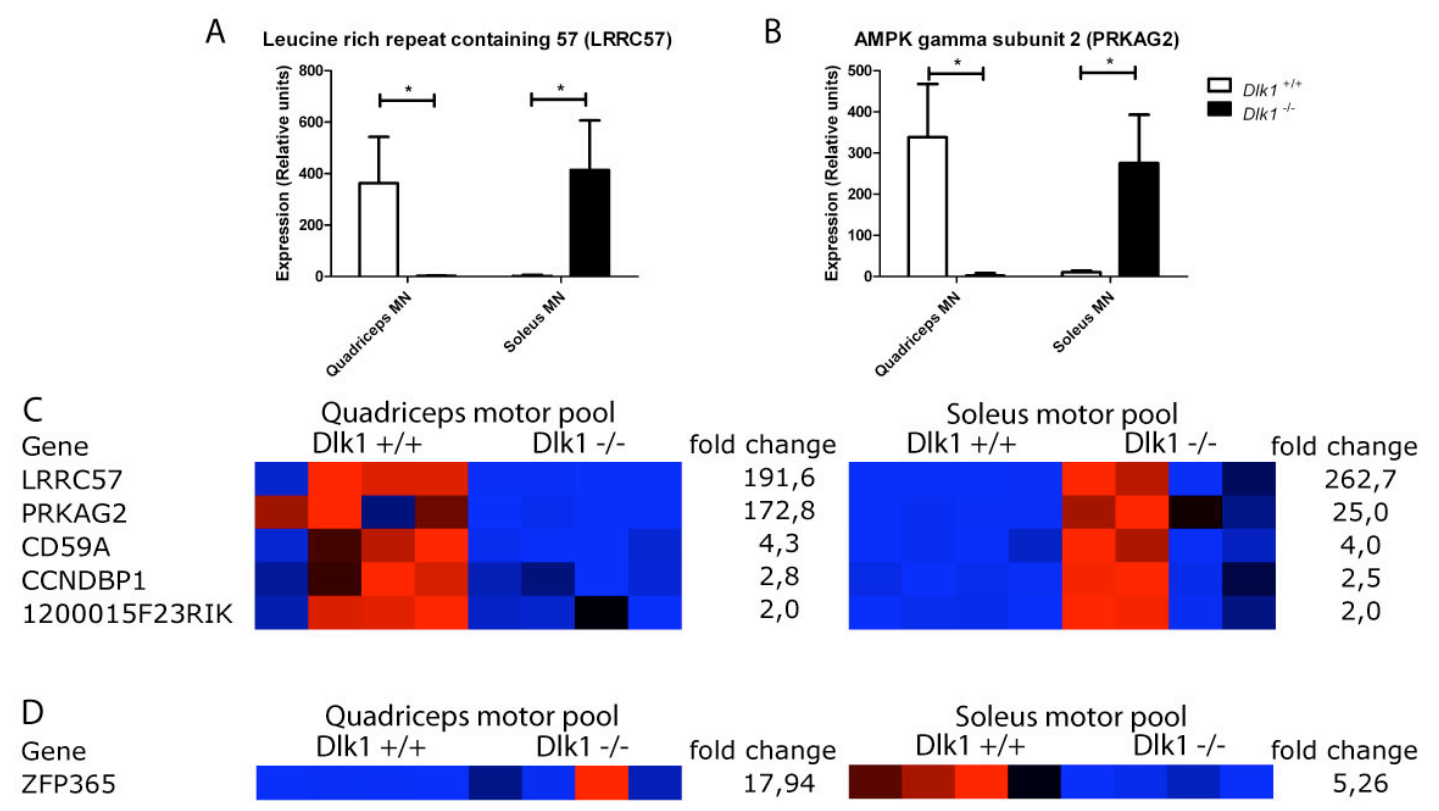

Fig. 5.30 Genes with switching expression in between Dlk1 wild type and knockout MNs Several genes expressed differentially in between fast and slow MNs in Dlk1 wild type animals switched their expression pattern and were expressed specifically in the other FMNTs in the Dlk1 knockout.

Subsuming the results from the microarray gene expression analysis in $D l k 1^{-/-}$fast and slow MNs, lack of Dlk1 expression is affecting expression of glucose metabolism related genes. Changes could be observed for genes like PRKAG2 (Protein kinase, AMP-activated gamma 2) a subunit of the AMP-activated protein kinase, PYBG (Brain-specific glycogen phosphorylase) and HK1 (Hexokinase 1). This could indicate a more general role of Dlk1 in the regulation of the cellular energy metabolism. Especially the strong shift in expression of the PRKAG2 gene is noticeable, as this gene is encoding a regulatory subunit of the AMPK (AMP activated protein kinase) a heterotrimeric protein playing a key role within the maintenance of the cells energy balance (Kemp et al. 1999). Mutations within the PRKAG2 gene are related to a cardiac glycogen storage disease termed WolffParkinson-White syndrome (Gollob et al. 2001 Folmes et al. 2009). Inflammatory response related genes like CTSC (Cathepsin C) and SERPINA3N (serine or cysteine peptidase inhibitor, clade A member $3 \mathrm{~N}$ ) were upregulated in Dlk1 -/- mice while the LCN2 (Lipocalin 2) gene expression was downregulated concurring with a suggested role of Dlk1 in these processes. Another variation considered expression of a gene involved in membrane lipid synthesis. The expression of AGPAT5 (1-acyl-snglycerol-3-phosphate acyltransferase epsilon) expression was significantly reduced in 


\section{Results}

Dlk $1^{-/}$fast but not slow MNs. While additional differences affect genes that may be involved either directly or indirectly in gene regulation like the NPAS3 (Neuronal PAS domain containing protein 3) that is upregulated in the knockout animals or the H2AFJ (H2A histone family J) that is downregulated in the knockout mice in Rectus femoris and Soleus MNs. Interestingly also expression changes in the NPAS3 and the PRODH (proline dehydrogenase) gene both associated with schizophrenia have been found (Pickard et al. 2005, Lang et al. 2007).

\subsubsection{Notch pathway gene expression}

Since the Dlk1 protein is a putative inhibitor of Notch signaling, the expression pattern of Notch pathway and Notch target genes was of particular interest. However, at the examined stage the expression of genes involved in that pathway did not show strong differences. The changes of the expression levels were minor and only significant for Hes1 and Notch1 in the Soleus innervating motor neurons (Fig. 5.31). Several other Notch related genes like Hes7 or Lunatic fringe (Lfng) were expressed in a sub threshold level.

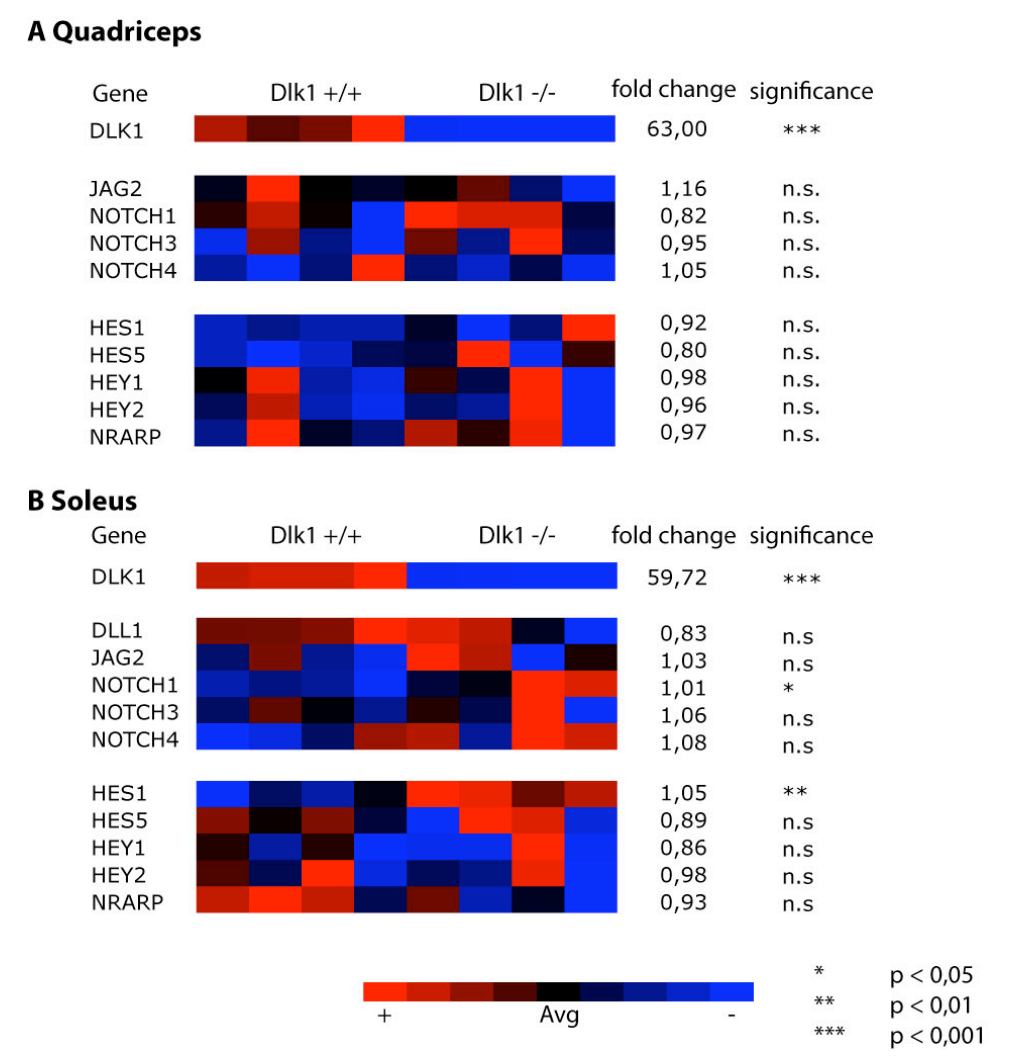

Fig. 5.31 Expression analysis of Notch pathway genes in Dlk1 wild type and knockout MNs Expression changes of Notch pathway genes were only slight and mostly insignificant in both the Quadriceps (A) and the Soleus (B) innervating motor neurons. 


\section{Results}

As there was only little or no change in Notch related gene expression in between Dlkl wildtype and knockout motor neurons it is unlikely that the Dlk1 function in motor neuron development is mediated by ort involved in Notch signaling at least at P4 stage. However, as the Delta/Notch signaling pathway is involved in neurogenesis in the spinal cord an earlier effect cannot be excluded especially regarding the expression of Dlk1 within cells of the pMN domain.

\subsection{Long-term transgene expression in the chick embryo spinal cord}

The chicken embryo has been shown to be a versatile model organism for developmental studies of the spinal cord development in general and the neuromuscular system in particular. A useful feature of the chick as precocial species is that the neuromuscular maturation is almost entirely completed during in-ovo gestation. Thus, we decided to test the ability of Dlk1 to directly influence MN properties upon forced expression in chick MNs in-vivo. However, manipulation of the chick spinal cord so far was limited to the initial stages of development due to loss of transgene expression as introduced DNA is degraded due to the transient nature of the transfection. As electrophysiological properties of different FMNTs are expected to consolidate in the later embryonic development it was necessary to create an expression systems that allows to stably express of a specific transgene in late developmental stages. To achieve this the tol 2 transposase system was employed, which already had been shown to work in chick embryos (Kawakami \& Shima 1999). 


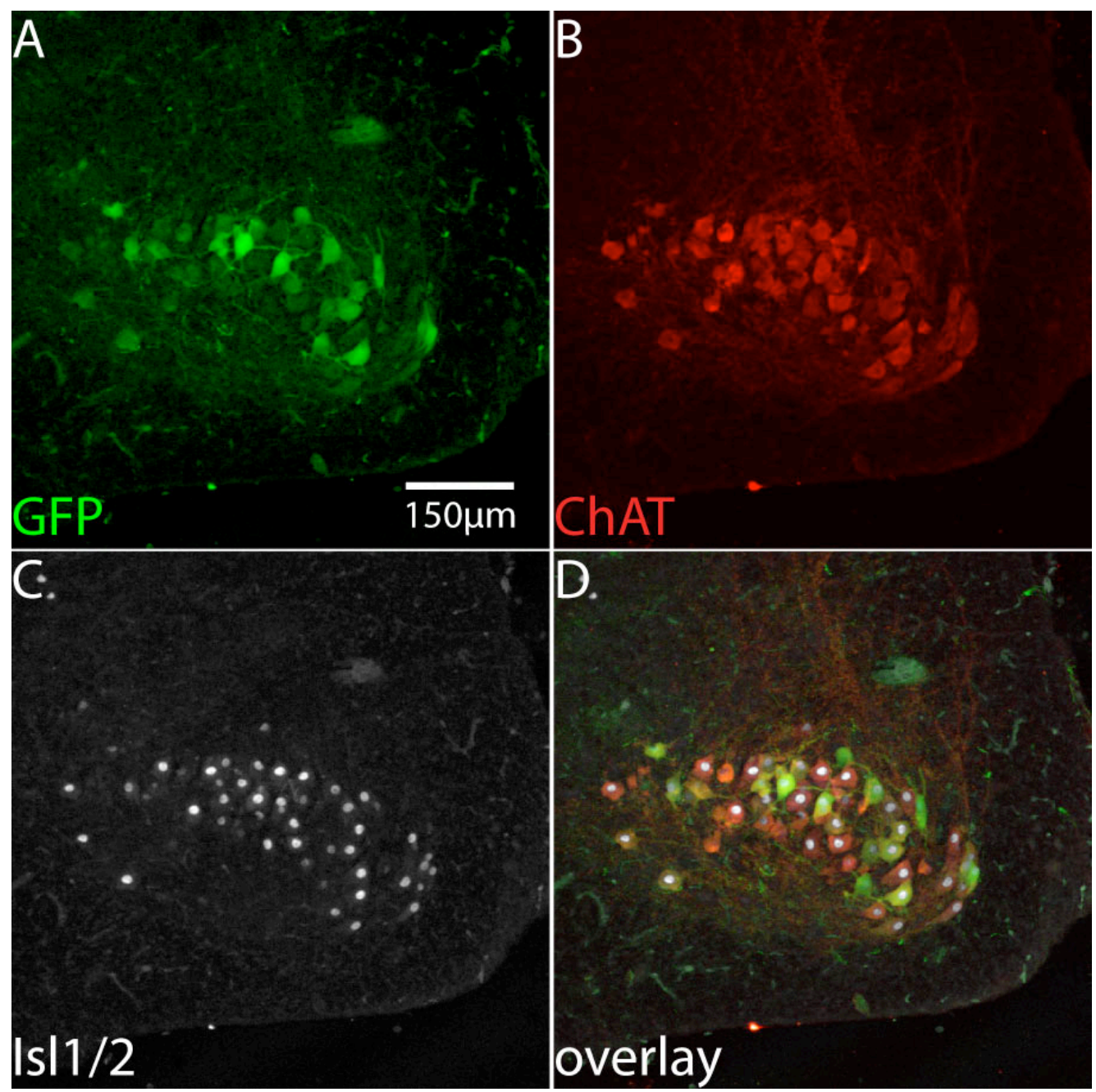

Fig. 5.32 GFP expression in motor neurons in chicken embryos at E18 GFP expression is monitored after electroporation with the long-term expression vector with the motor neuron specific Hb9-CRE driver plasmid and the transposase coding pCAGGS-T2TP plasmid. GFP expression (A), anti-ChAT immunostaining (B), antiIsl1/2 immunostaining (C), overlay picture (D).

The transposase mediated long-term expression vector system (see also Methods 4.8) designed in this work has proven to allow transgene expression until the latest developmental stages of the chick embryo up to embryonic day that equals 15 days post electroporation (Fig. 5.32) due to stable genomic integration. Moreover the combination of the ubiquitous CAGGS promoter together with specific transgene activation mediated by coelectroporation of a Cre driver plasmid makes the system independent of promoter strength or activation (Voigt et al. 2010). Upon activation by Cre the transgene will be continuously expressed at a high level in all cells that have been specifically expressed the Cre driver promoter (Fig. 5.33). Thus, the system allows a lineage tracing of cell that have expressed a specific marker gene at an early stage of development. 


\section{Results}

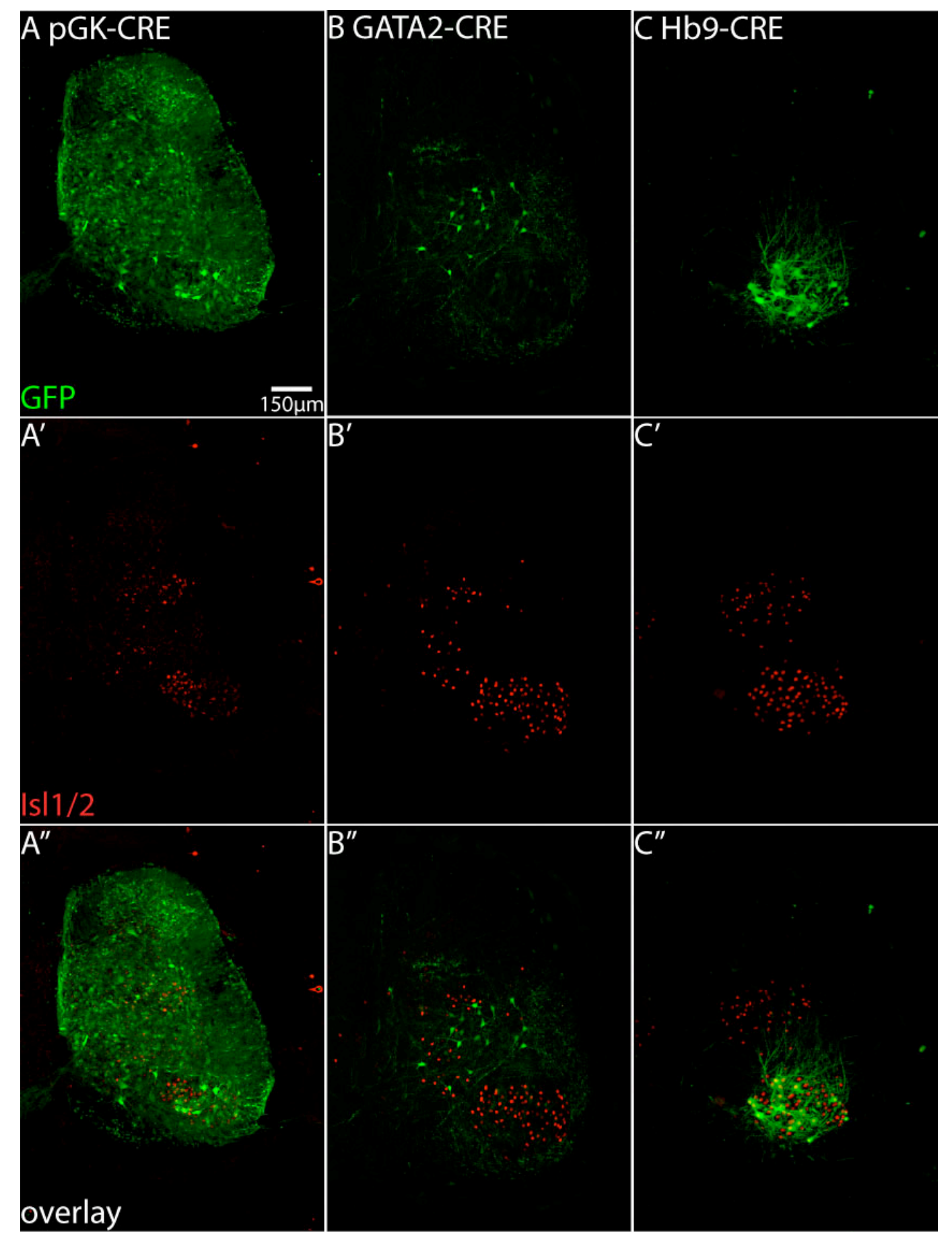

Fig. 5.33 Cell type specific long-term transgene expression GFP Expression after coelectroporation of phosphoglycerate kinase 1 (A), Hb9 or GATA2 promotor driven CRE. Immunostaining with Isl1/2 as topographic marker for $\mathrm{MN}$ and dorsal interneurons (A', B', C'). Overlay picture (A', B', C', ). 


\section{Results}

\subsection{Effects of Dlk1 on the electrophysiological properties of chicken motor neurons}

Among the defining features of FMNTs are their inherent electrophysiological properties that mediate the specific activity outputs matched to the physiological properties of the muscle fibers they innervate. Among these are passive membrane properties, such as input resistance (which is typically low in fast MNs), as well as active properties, such as the rheobase (which is higher in fast compared to slow MNs). The newly designed vector system was applied to express a chicken Dlk1-GFP fusion protein in late chicken embryo motor neurons in order to analyze changes in FMNT specific electrophysiological parameters. Chor Hoon Poh performed these studies in our lab.

Dlk1-GFP expressing cells showed a significant decrease in input resistance and a concomitant albeit borderline non-significant $(p=0.07)$ increase capacitance. In addition, the rheobase was increased, indicating that a stronger stimulation is necessary to evoke activity in these cells. Although the changes for capacitance and rheobase are not significant based on the number of analyzed cells so far these changes suggest that the Dlk1 expressing cells are shifted towards a faster FMNT electrophysiological profile. In order to analyze a potential cell non-autonomous function of Dlk1 also MNs in direct neighborhood of Dlk1 expressing MNs have been analyzed by patch clamp. So far no changes in their electrophysiological characteristics were observed, suggesting that Dlk1 exerted its activity on MN properties in a cell-autonomous manner. Ongoing and future studies will address possible differential impacts on FMNT status when Dlk1 activity is selectively manipulated in MN progenitors or postmitotic MNs. 


\section{Results}

A
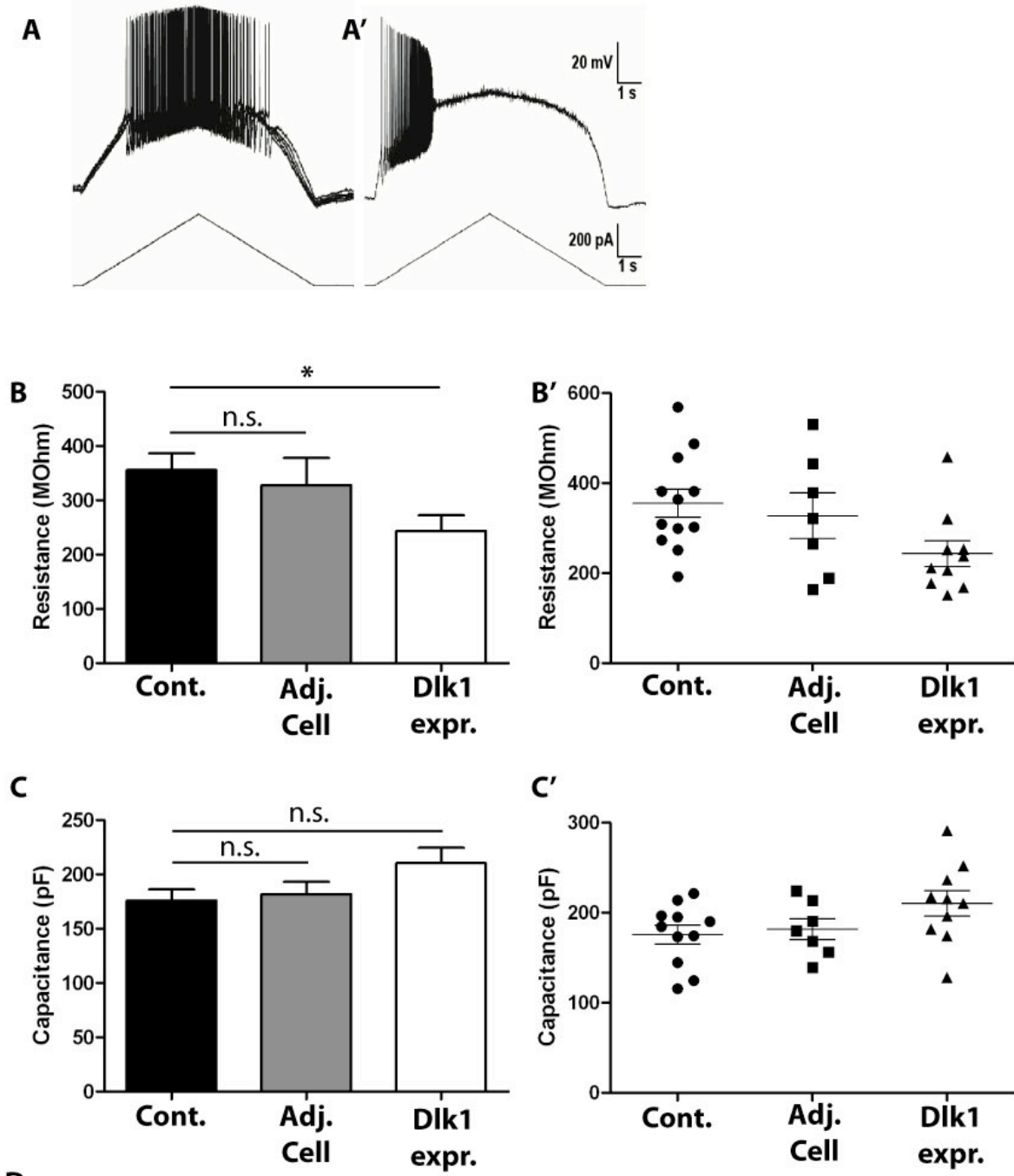

C'
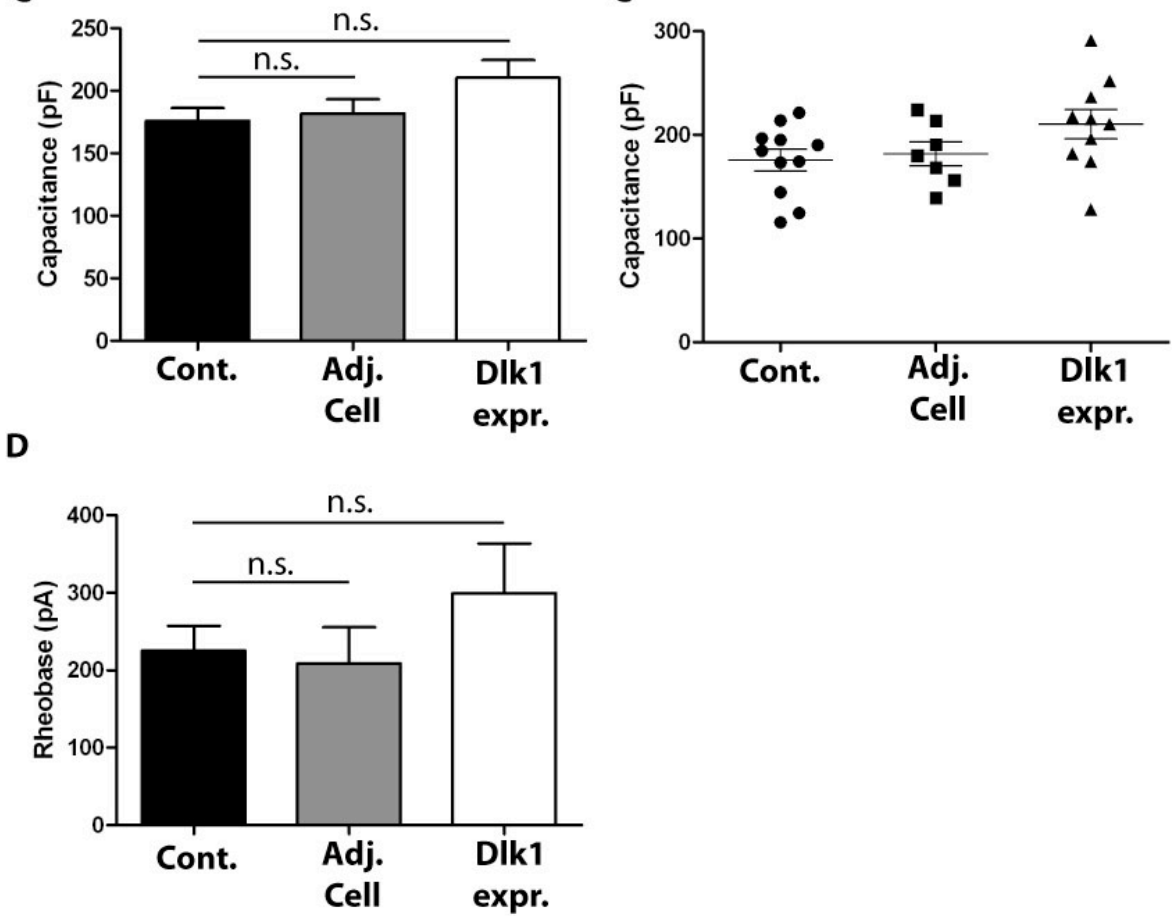

Fig. 5.34 Electrophysiological analysis of chick MNs overexpressing Dlk1 E12 to E14 chicken MNs have been electrophysiologically characterized by patch clamp recordings in order to monitor the effects of Dlk1 expression. Three groups of cells were analyzed control cells (Cont.) were MNs electroporated with a GFP only expressing construct. Dlk1 expressing cells (Dlk1 expr.) were MNs electroporated with the chicken Dlk1-GFP fusion protein and adjacent cells (Adj. Cell) are MNs in direct vicinity of Dlk1-GFP expressing cells. Motor neurons could be discriminated due to their bistable behavior (A) from other neurons (A'). The cells were analyzed for input resistance (B, B'), capacitance (C,C') and the rheobase (D). 



\section{Discussion}

\section{Discussion}

The correct conduction of body posture and locomotion is highly dependent on proper function and integrity of the different FMNTs. Many aspects of the initial steps of MN differentiation have been elucidated over the past years. This has lead to a vast knowledge about factors involved in formation of motor neuron progenitors, as well as postmitotic motor neuron populations (Briscoe \& Ericson 2001, Dasen \& Jessell 2009). However, it remains unknown, how MNs ultimately become specified into different FMNTs. Despite their pivotal functional role and relevance not only within a discrete set of neuromuscular disorders like ALS but also in general ageing processes, our knowledge about FMNTs still relies almost entirely on physiological and morphological observations (Hashizume et al. 1988, Kadhiresan et al. 1996, Frey et al. 2000, Pun et al. 2006, Hegedus et al. 2007, Kanning et al. 2010).

\subsection{General considerations regarding the differential analysis of FMNT populations}

During this work an experimental setup was designed to grant access to different motor neuron populations by using the retrograde in vivo transport of the cholera toxin B subunit (Fujinaga et al. 2003). Based on that approach it was possible to distinguish between the different $\mathrm{MN}$ populations innervating the mouse hind limb muscles Rectus femoris and Soleus, which are predicted to be enriched in either fast or slow MNs. Via analysis of the motor neuron cell soma size distribution it was possible to indicate the predominant presence of the different FMNTs within the two motor pools at postnatal day 4 and 10 (Burke et al. 1977). As the cell size differences were found to be apparent at these early stages it is suggested that molecular differences between the FMNTs were already established. This also concurs with the findings of selective innervation of fast and slow muscle regions in the avian muscles (Rafuse et al. 1996). Indeed, the results obtained in this thesis revealed a differential gene expression in MNs innervating either fast or slow muscle fiber types. In addition to providing the first mechanistic insights into the specification of MNs into different FMNTs, the gene signatures of MNs exhibiting a high degree of resistance towards MNs pathologies may offer novel diagnostic markers or therapeutic targets for MN 


\section{Discussion}

diseases (Kojima et al. 1989, Gizzi et al. 1992, Carvalho et al. 1995, Mannen 2000, Kaminski et al. 2002).

\subsection{Towards the identification of further functional motor neuron type specific genes}

Since the key questions regarding FMNT development and specification are still unanswered the molecular markers evaluated in the present work will provide an essential entry point for gaining deeper insights into these processes. While the present study represents the first systematic and stringent examination of FMNT gene expression signatures, two recent studies independently suggested four gene markers linked to fast and slow MNs (Chakkalakal et al. 2010, Enjin et al. 2010). Apart from SV2a described by Chakkalakal et al., for the three other markers the actual specific expression in slow or fast MNs remains to be confirmed. With available markers not only for FMNTs but also for alpha and gamma class MNs provided by the studies of Friese et al. and Shneider et al. the possibility to monitor formation and development of these cells in health and disease at the molecular level may become available in the near future (Friese et al. 2009, Shneider et al. 2009).

However, up to now it is not possible to pinpoint the varying properties of the different FMNTs towards one of the identified genes. Also, it could not be clarified finally how many different FMNTs actually exist. This considers the group of fast FMNTs, as it has still to be clarified whether fast type FF, FI and FR FMNTs are distinguishable from each other at the molecular level. Hence, there are further studies necessary to not only access novel marker genes but also to define key factors underlying FMNT specification and functional properties. In order to facilitate these examinations a workflow schema is introduced based on the results of this work (Fig. 6.1). It is proposed that new potential FMNT specific candidate genes should be verified according to this workflow protocol and also stringently correlated to already known FMNT markers (Fig. 6.2). 


\section{Discussion}

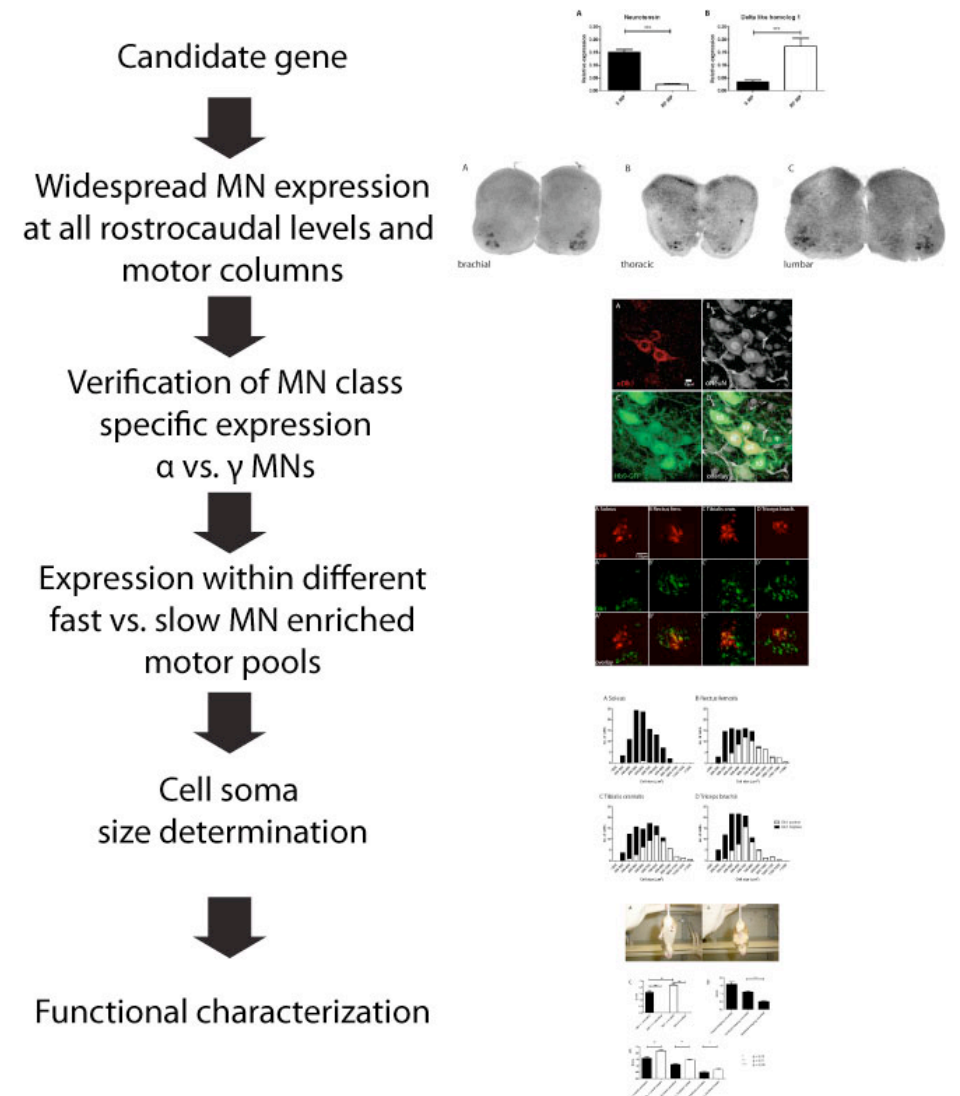

Fig. 6.1 Workflow for the identification of FMNT specific genes

Initially, it is necessary to elucidate if the putative FMNT specific gene is expressed in the expected "salt and pepper" fashion in MNs throughout the spinal cord. The Allen Brain Atlas has proven to be a valuable resource not only for an initial examination of the expression pattern but also for providing workable in-situ hybridization probes (Lein et al. 2007). In the next step it needs to be clarified, whether the candidate gene can be detected within all rostrocaudal levels and motor columns. Thereby, motor pool or region specific expression like observed for the Hox cluster transcription factors, or the cadherin class cell adhesion molecules can be excluded (Price et al. 2002, Dasen et al. 2005, Dalla Torre di Sanguinetto 2008). As gamma MNs make up one third of the overall $\mathrm{MN}$ population and also represent a considerable portion of the small size MNs it is advisable to examine expression within the different MN classes. Therefore, based on the results of this work and the results by Friese et al., this can be achieved by correlation with the alpha MN marker NeuN (Friese et al. 2009). Unfortunately the population of beta MNs is not accessible so far. Thus, it is not clear whether this putative MN population innervating intra- and extrafusal muscle fibers simultaneously, may be assigned towards the alpha or the gamma MN class according to the methods and molecular markers available up to 


\section{Discussion}

now. Also it is still controversial whether this is actually a discrete MN population. Subsequently, correlation of a novel marker with selectively labeled MNs from motor pools enriched in fast or slow MNs based upon muscle fiber composition is a good indicator for assigning gene expression to a distinct FMNT. With this method one has to be cautioned, however that MN proportions obtained in such analyses can only be correlated in a general manner to muscle fiber composition as fast motor units generally contain more muscle fibers compared to slow ones (Burke \& Tsairis 1973). Finally, the expression pattern can be correlated with MN soma size, which is the most laborious part of the verification process due to the large number cells that have to be examined in order to make a qualified statement.

Such an analysis has to account for the fact that quantifications of cell sizes are difficult to compare between different labeling methods or markers. In this work the average soma size of a gamma MNs defined by low or absent expression of NeuN was determined to be $371.4 \mu \mathrm{m}^{2}$ at $\mathrm{P} 4$, while Shneider et al. state a cell soma size of $334 \mu \mathrm{m}^{2}$ for gamma MNs at P20 and Friese et al. propose gamma MNs to have a size of $232.4 \mu \mathrm{m}^{2}$ at P21 (Friese et al. 2009, Shneider et al. 2009). A similar observation was made by comparison of the cell size of Dlk1 positive fast LMC MNs with that of Calca positive fast MNs (Enjin et al. 2010). In this work the Dlk1 positive MNs had an average soma size of $938 \mu \mathrm{m}^{2}$ quantified on the GFP positive area and $744 \mu \mathrm{m}^{2}$ based on the CTxB positive area in retrogradely labeled Rectus femoris MNs in P4 mice. While Enjin et al. claimed the size of a Calca positive fast MN to be $351 \mu \mathrm{m}^{2}$ based on Calca specific in-situ hybridization in P0 mice. It is likely that the different subcellular localization of the molecular markers used for defining the examined MN populations might be the reason for this observation. Also, varying tissue fixation, dehydration and labeling protocols as well as differing methods of size quantification are likely to contribute for such variations. Therefore, cell soma size quantifications should only be used to yield information on relative cell sizes and have to be carried out strictly according to the same standardized protocols to maintain consistency of the results at least as long as they are based on a predetermined marker and labeling technique.

Another more direct way to address FMNT specific gene expression is to link electrophysiological characterization of specific MNs with a subsequent immunohistological analysis. This was attempted by Enjin and colleagues. However, such an analysis is highly elaborate and should thus be restricted to genes that are 


\section{Discussion}

likely to be FMNT specific according to previous analysis. The most stringent method yet to be applied would be to use identified and confirmed FMNT specific markers to genetically "tag" fast or slow MNs, via gene targeting or transgenic expression of a fluorescent protein. This also would allow direct electrophysiological characterization of genetically identified FMNTs.

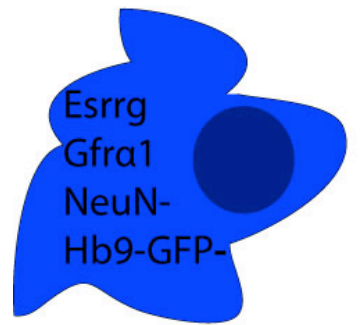

Y MN

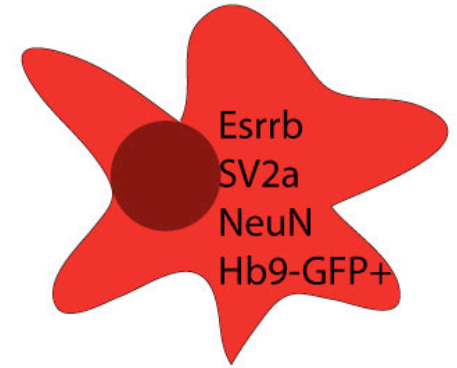

aS MN

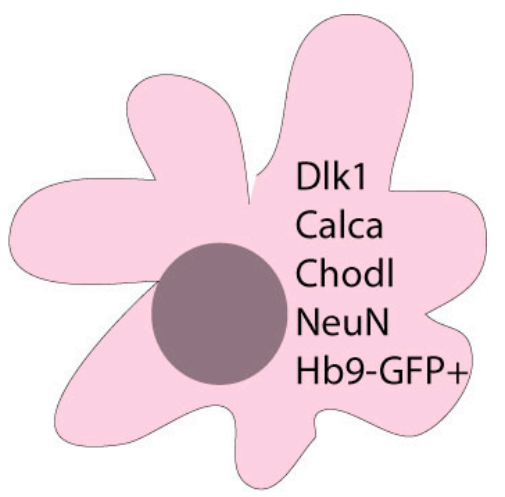

aF MN

Fig. 6.2 The known molecular markers of motor neuron classes and types A general overview about the currently available or proposed MN class and FMNT markers.

\subsection{The expression analysis of the Dlk1 protein in motor neurons and other tissues}

Since the Dlk1 protein was confirmed to be expressed in fast MNs in the frame of this work the expression of Dlk1 in the spinal cord in general and in motor neurons in particular was examined. During this work the Dlk1 expression could be correlated with large soma size MNs innervating predominantly fast muscle fibers. Dlk1 was initially found to play a negative role in the process of adipocyte differentiation by keeping fibroblast cells in an undifferentiated state (Smas \& Sul 1993, Wang et al. 2006). Furthermore, a role in muscle development and regeneration is suggested (Davis 2004, Murphy et al. 2005, Waddell et al. 2010). Also Dlk1 is influencing B cell differentiation and maturation (Raghunanden et al. 2008). Other publications moreover suggest a role within the development of dopamineric neurons (Christophersen et al. 2007, Bauer et al. 2008). These studies commonly propose a role for Dlk1 that is mainly restricted to embryonic development, differentiation and regeneration since expression of the protein was not detectable any more in many cell types after reaching a mature state. 


\section{Discussion}

\subsection{The role of Dlk1 during motor neuron development}

Despite the assumption that muscle fibers are initially selectively innervated by their associated FMNTs, maturation and maintenance of motor neurons and muscle fibers is subjected to be based on a dynamic mutual regulation (Rafuse et al. 1996). So far no specific recognition mechanisms, or molecules for FMNT/muscle fiber type matching and specific for FMNTs proper have been elucidated.

The Dlk1 gene was identified to be present in motor neuron progenitors from E10.5 and in some MNs from mouse E12.5 stage onwards. As there are no detectable levels of Dlk1 in MNs directly after they have exited the cell cycle, it can be speculated that the early Dlk1 expression in MN progenitors is playing an independent role from the subsequent expression in postmitotic MNs. Thus, this makes it necessary to differentiate between an early progenitor-specific and a late FMNT-specific function of Dlk1. So far, it is speculative whether this early function depends on, or is mediated by alternative protein isoforms, or specific protein interaction partners like the Notch receptor proteins.

E12.5 is an embryonic stage in which almost all MNs have already been created although some scattered late born cells can be observed. It remains to be determined whether the prenatal Dlk1 expression in MNs is found within the same cells that have been confirmed as large soma size fast MNs in later stages of development. To clarify this it would be necessary to lineage trace Dlk1 positive cells e.g. by a tamoxifeninducible reporter line expressing CRE-ERT2 under the control of a specific Dlk1 promoter. However, at E12.5 generally MNs have not reached their muscular targets yet, and therefore the expression of a FMNT specific marker would suggest the process of FMNT specification being independent of NMJ formation and retrograde signals provided by muscle fibers (Lin et al. 2008). This observation is consistent with the results of Enjin et al. suggesting Esrrb to be expressed within E11.5 MNs. However, Chakkalakal et al. are claiming that transgenic expression of PGC1 $\alpha$, which is shown to convert muscle fibers towards slower type I characteristics, is able to promote expression of the slow MN marker SV2a. But it has to be clarified if this effect is related to neuronal plasticity rather than the specification of slow MNs. To further investigate the role of motor neuron/muscle fiber interaction in development and neuronal plasticity it would be feasible to examine the expression of Dlk1 and other FMNT specific genes in mouse embryos lacking muscle fibers, or for instance 


\section{Discussion}

by myocytes ablation by selective toxic transgene expression. This can be readily achieved using MyoD-Cre or Myogenin-Cre mice together with ROSA-eGFP-dta mice (Chen et al. 2000, Ivanova et al. 2005, Li et al. 2005).

The results obtained so far raise the question regarding mechanisms or factors that are determining FMNT fate in developing MNs. According to the current state of research it cannot be determined if FMNTs are generated from a certain population of progenitors subdividing the pMN domain into a $\mathrm{pMN} \alpha 1$ and a $\mathrm{pMN} \alpha 2$ region. These regions could form in response to the ventral to dorsal gradient of Shh like it has been shown for the other progenitor domains of the ventral spinal cord (Jessell 2000, Shirasaki \& Pfaff 2002). Another hypothetical possibility is the generation of the different FMNTs in temporally segregated waves. On the other hand, it is known that the $\mathrm{MNs}$ forming the $\mathrm{LMCl}$ are generated later than $\mathrm{LMCm}$ neurons, and both of these LMC subcolumns contain the full spectrum of FMNTs (Hollyday \& Hamburger 1977, Price \& Briscoe 2004). Thus, such a time dependent mechanism seems to be less likely. It is also thinkable that MNs acquire their cell fate due to cell-to-cell interactions, or exogenous signals similar to the Sonic hedgehog gradient postmitotically.

The scattered expression of Dlk1 within MNs is maintained at least until P10 FMNT, but is completely extinguished by postnatal day 20 (Fig. 6.3), while the fast FMNT specific expression is confirmed for the P4 and P10 stage. Thus, Dlk1 is not expressed any more in mature MNs and it will be necessary to elucidate in which ways this transient expression is significant for maturation of FMNTs. Unfortunately, these findings exclude the use of Dlk1 as marker to analyze the properties of fast MNs in later stages especially within neuromuscular diseases. Since Dlk1 is activated as part of the process of muscle regeneration also in adult mice (Waddell et al. 2010) it remains to be elucidated whether motor neuron damage caused by progressive neuromuscular diseases, inflammation or injury might reactivate Dlk1 in mature MNs and if this effect is restricted towards a particular FMNT. 
Developmental stage
E10.5
E12.5
E15.5
E18.5
P4 P10
P20

Motor neuron generation and maturation

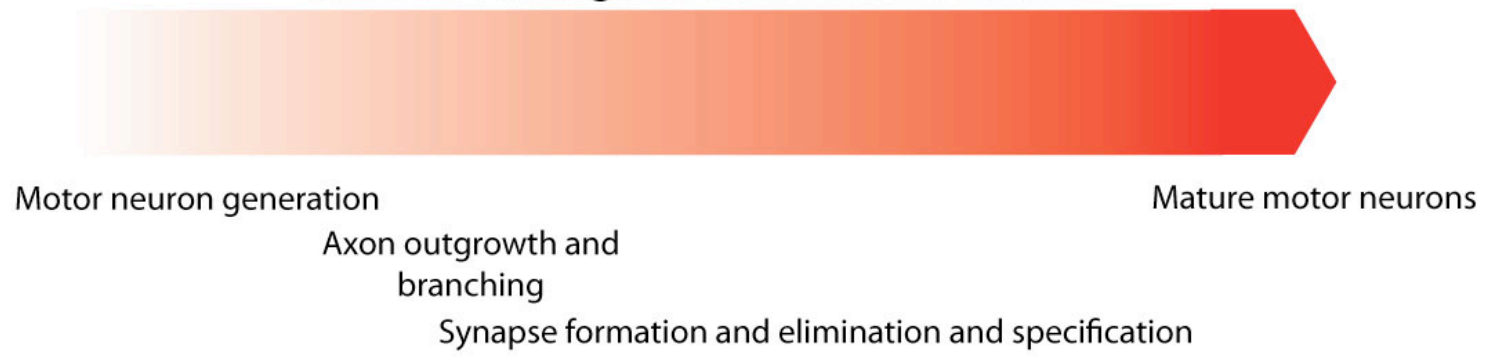

Dlk1 expression

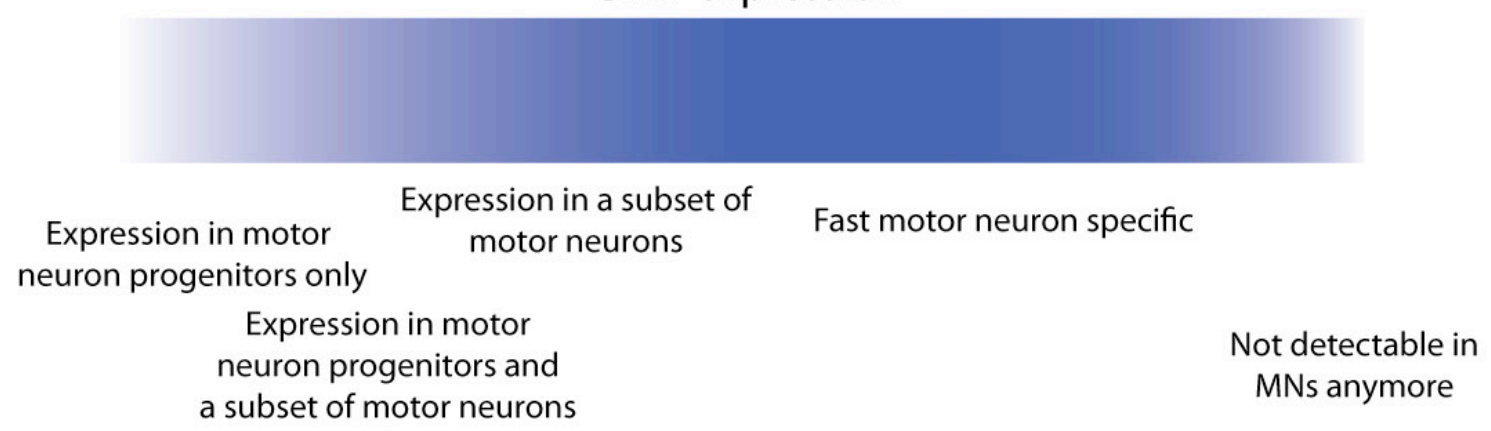

Fig. 6.3 The expression of Dlk1 in different stages of motor neuron development Dlk1 is initially present within motor neuron progenitors but not within postmitotic MNs. However it is expressed in a subset of MNs from E12.5 onwards. The Dlk1 expressing cells could be confirmed as fast MNs at P4 and P10. From P20 onwards Dlk1 was not detectable in MNs anymore.

\subsection{Behavioral observations in the Dlk1 knockout mouse model}

The Dlk1 mouse line used in this work was originally designed by Raghunandan et al. and showed the complete absence of Dlk1 mRNA and protein as verified by in-situ hybridization analysis, immunostainings and microarray expression analysis in this study (Raghunundan et al. 2008). Therefore, potential dominant negative effects or a partial rescue of the phenotype caused by expression of a truncated form of the protein could be excluded. The mice did not show the very prominent defects in eye development or the high early postnatal mortality of 50\% described for the mouse line of Moon et al. (Moon et al 2002). Hence, these previous observations may be related to the genetic background, or to defects carried over from the embryonic stem cells used to generate the mouse line.

In our hands, these animals deficient for the Dlk1 gene have shown several abnormalities putatively linked towards a role of the protein in the neuromuscular development. Most prominent was the fore and hind limb clasping behavior exhibited 


\section{Discussion}

upon tail suspension, which significantly increased after the animals were subjected towards a short high intensity running exercise. The clasping phenotype was found in several mouse model systems of neurological diseases like for Huntingtons disease, ALS or Rett syndrome (Mangiarini et al. 1996, Guy et al. 2001, Hansson et al. 2003, Bruestle et al. 2009). Thus, a certain neuromuscular impact of Dlk1 loss is indicated. The interesting observation of an increased clasping after a short intensity could indicate an impaired ability of Dlk1 knockout mice to cope with neuromuscular stress. This could either be a sign for a defective signal transduction in the neuromuscular system that might have been caused by a dysfunctional energy metabolism or increased oxidative stress. On the other side long-term low intensity treadmill running or voluntary wheel running might be able to reduce clasping behavior as this was reported for Huntington's disease model animals (Pang et al. 2006). Furthermore, $D l k 1^{-/-}$animals exhibited significantly lower performance in running endurance and grip strength experiments compared to wild type controls.

Indeed, if the clasping phenotype or the other impairments observed are related to the Dlk1 function in MNs cannot be finally confirmed, as the Dlk1 knockout examined in this study is systemic. Especially the defects in muscle regeneration reported by Waddell et al. might cause or enhance such a phenotype especially regarding a potential interplay with the role of Dlk1 in inflammation processes (Waddell et al. 2010). Although there were no such observations published in this report for the conditional knockout animals deficient for muscular Dlk1 expression. Moreover, these results are debatable as besides fast motor neurons, motor neuron progenitors and muscle satellite cells Dlk1 is also highly expressed in the developing muscle fibers and lack of Dlk1 enhances fat cell development resulting in a higher body weight (Floridon et al. 2000, Moon et al. 2002, White et al. 2008, Waddell et al. 2010). Therefore, increased body weight, or non-physiological ratios of body fat towards muscle mass resulting in a non-physiological metabolic status might cause or contribute to the observed phenotype. Also, these experiments were performed in mice with an age of four to six month, while Dlk1 is not detectable in MNs at P20 and onwards. This raises the question whether and how the lack of Dlk1 protein function during earlier stages of MN development might cause such long-term effects. Thus, these findings will have to be verified based on a conditional knockout mouse line specifically deficient for Dlk1 expression in MNs or muscle fibers. Hence, the 


\section{Discussion}

recently described conditional knockout mouse line developed by Waddell and colleagues will be useful and has been acquired by our laboratory lately.

\subsection{The effects and mechanisms of Dlk1 at a molecular level}

So far, the molecular mechanisms of Dlk1 mode of action remain controversial. While evidence for an involvement in the Notch signaling pathway was reported by Baladron et al. and Bray et al., such interactions have been ruled out by Wang and colleagues (Baladron et al. 2005, Bray et al. 2008, Wang et al. 2010). A possible mode of action that could also be relevant for Dlk1 was recently suggested for Deltalike 3 protein (D113) in the developing presomitic mesoderm (Chapman et al. 2010). Here D113 appears to intercept Notch1 on the way to the cell membrane, and targets it for lysosomal degradation. This results in Notch being not presented at the cell surface any more, causing it to be unavailable for activation or repression of downstream target genes. These findings suggest that besides the classical posttranslational modifications of Notch involving cleavage of the Notch intracellular domain there might be other mechanisms to fine tune this pathway in a specific tissue, cellular and developmental context. However, the results shown in this thesis work indicate that loss of Dlk1 expression in postnatal MNs has only minor, or insignificant effects towards Notch downstream target genes such as Hes1. This suggests a Notchindependent role of the Dlk1 protein, such as proposed by Wang et al., at least for postmitotic MNs. However, compensatory mechanisms may be thinkable that may overcome the lack of Dlk1 and thereby normalize Notch signaling in the absence of the protein. Also a Notch-mediated role of Dlk1 in MNs cannot be excluded taking into account the early Dlk1 expression in MN progenitors at E10.5 and E12.5. Especially as the Notch pathway is involved in the specification of V2 interneuron populations directly after they have been generated within the ventricular zone at E11.5 (Peng et al. 2007).

Gene expression analysis of Dlk1 knock out mice revealed a set of genes that switch in their expression between the motor pools in absence of Dlk1. Such an unusual behavior might be explainable through a compensatory mechanism mediated by the Dlk2 or EGFL9 gene. Dlk1 and Dlk2 are sharing a high grade of amino acid homology within the N-terminal EGF-like repeat containing extracellular domain, while the C-terminal intracellular region is completely different (Nueda et al. 2007). 


\section{Discussion}

So far the knowledge about Dlk2 has remained rudimentary, however the results by Nueda et al. suggest that Dlk1 and Dlk2 genes are able to influence each others expression. While reduction of Dlk1 expression by siRNA knockdown increases expression of Dlk2, overexpression of Dlk2 decreases Dlk1 expression in cell culture. In MNs of Dlk1 knockout animals however the expression levels of Dlk2 are not increased at P4, therefore such a kind of feedback inhibition might not be present or the mechanism will act at a different stage of development. Dlk2 is expressed at low and similar levels in both fast and slow MNs according to the microarray experiments and the expression is altered in Dlk1 knockout MNs. Therefore, it may be speculated that Dlk2 mediated effects normally suppressed by Dlk1 activity in fast MNs might occur in $D l k 1^{-/-}$MNs and alter the gene expression profile.

Among the genes that showed a switch in their expression between Soleus and Quadriceps MNs in Dlk1 knockout animals, PRKAG2 or AMPK gamma subunit 2 was of particular interest. The expression levels of genes that are expected to be involved in electrophysiological properties such as ion channels are not alter in $\mathrm{Dlk} \mathrm{K}^{-/-}$ MNs. Thus, a different molecular mechanism may account for the activity of Dlk1 in MNs. PRKAG2 is the regulatory unit of the AMPK (AMP-activated protein kinase) protein that is known to be a pivotal energy sensor in the cell. The gamma subunit of the AMPK heterotrimer modulates AMPK activity by activation of the catalytic alpha subunit in presence of high AMP concentrations. AMPK has also been shown to regulate ion channels e.g. the TREK1 and TREK2 potassium channels, or voltage gated sodium channels by phosphorylation (Light et al. 2003, Evans et al. 2009, Kréneisz et al. 2009). Thus, Dlk1 may influence electrophysiological properties of MNs via AMPK signaling. Besides PRKAG2, PRKAG1 is also found in MNs while a third form termed PRKAG3 is not present. The influence of Dlk1 loss not only towards the expression level of PRKAG2, but also on PYGB (brain-specific glycogen phosphorylase) and HK1 (Hexokinase 1) suggests a role for Dlk1 regarding the metabolic regulation within MNs, possibly by centrally regulating AMPK signaling.

The finding of inflammation related genes like SERPINA3N, CTSC or LCN2 to be differentially regulated between $\mathrm{Dlk}^{+/+}$and $\mathrm{Dlk} 1^{-/-}$MNs may be not surprising, as Dlk1 has been implicated in inflammatory processes in other tissues as well. The finding of increased levels of SERPINA3N expression was of particular interest, as increased SERPINA3N or alpha 1 antichymotrypsin levels have been detected in ALS patients (Lim et al. 1996) Also other findings indicate a possible role in the disease 


\section{Discussion}

progression of Alzheimer's disease as SERPINA3N can induce tau phosphorylation, and polymorphisms in the promoter region of the gene have been correlated with age of disease onset and duration of disease progression (Licastro et al. 2005, Padmahaban et al. 2006). Regarding the increased levels of such inflammatory proteins within MNs an analysis of spinal cords of $D l k l^{-/}$animals regarding astrogliosis in order to examine potential neuronal damage is suggested.

\subsection{Effects of Dlk1 on electrophysiological motor neuron properties}

Forced overexpression of chicken Dlk1 seems to alter MN electrophysiological properties towards a faster phenotype, although the findings so far are only preliminary and further control experiments are necessary. The input resistance of Dlk1 overexpressing cells was found to be decreased, which could indicate a shift towards a faster FMNT profile (Zengel et al. 1985, Gardiner 1993). In addition, the rheobase, was found to increase. This would ssuggest such a MN would be recruited later upon stimulation, which would be consistent with a shift towards faster FMNT properties (Zajac \& Faden 1985, Zengel et al. 1985). These findings suggest a direct activity of Dlk1 in FMNT specification. This raises the question how Dlk1 could affect physiological properties of FMNTs. As already mentioned AMPK is able to regulate ion channels so changes in AMPK subunit expression levels might induce alterations in activity of such channels (Light et al. 2003 Kréneisz et al. 2009). Input resistance and capacitance are considered passive membrane properties that are largely independent of ion channel activity, which have been characterized to be differential between fast and slow FMNTs. Alterations in cell size or membrane composition might induce variations in such parameters that might be due to changes in expression levels of proteins relevant for membrane composition like AGPAT5. So far theses findings have occured only in a cell autonomous fashion, implicating a cell autonomous function for Dlk1 in MNs. Certainly, there are further experiments necessary to clarify the precise mode of action of Dlk1 as well as that of potential effectors regulated by Dlk1. Thus, it is suggested to examine the effects of the different isoforms of murine Dlk1 towards electrophysiological MN properties as well as the effects of genetically engineered forms that are mandatory membrane bound or secreted. Also the effects of genes that are differentially expressed within Dlk1 knockout and wild type animals are of particular interest as they might give insights 


\section{Discussion}

towards the actual Dlk1 pathway in MNs. Especially it should be clarified whether PRKAG2 overexpression is able to reproduce effects observed in Dlk1 overexpressing MNs.

\subsection{A possible role of Dlk1 in motor neuronal pathology}

Since Dlk1 gene expression is not present any more in mature motor neurons effects on susceptibility towards $\mathrm{MN}$ diseases would be indirect and will likely concern the specification status of FMNTs. As mentioned before fast MNs are degenerating faster not only in mouse models of ALS but also in human patients (Dengler et al. 1990, Pun et al. 2006). The reasons for these findings are unknown so far as well as for the occurrence of most ALS cases. However it might be possible that there are metabolic differences between the FMNTs reflecting these observed in muscle fiber types. Such a relationship has been proposed by Pearson \& Sickles as Soleus MNs as well as associated muscle fibers have shown a reduction in NADH tetrazolium reductase activity upon ablation of Soleus synergistic muscles (Pearson \& Sickles 1987). Another aspect suggesting a relevance of specific metabolic imposition in the occurrence of ALS is the significantly elevated incidence of the disease amongst soccer players (Chiò et al. 2002, Chiò et al. 2005, Wicks et al. 2007). Besides other possible explanations like the use or abuse of pharmaceutical drugs, microtraumatas or exposure to toxic substances originating from pesticides or fertilizers, the specific requirements of this sport towards the neuromuscular system might lead to a surcharge that promotes $\mathrm{MN}$ damage. Considering these findings a factor like Dlk1 that is influencing metabolic aspects within $\mathrm{MN}$ development might also have a longterm impact on the general metabolic profile of the cells thus influencing disease susceptibility.

\subsection{Outlooks}

The recent results by Enjin et al., Chakalakal et al. and this work have finally proven the assumed molecular variances suggested by morphological and electrophysiological differences between the FMNTs. Therefore they have paved the way to a more profound understanding of the processes that underlie development and functionality of FMNTs as well as the mechanisms involved in their inherent susceptibility or resistance towards motor neuron diseases. 


\section{Discussion}

The ongoing work in our lab includes the generation of bacterial artificial chromosome based transgenic mouse lines to facilitate selective genetic labeling of the different FMNTs by the expression of fluorescent marker proteins. These mouse lines will allow tracking of individual FMNTs during the developmental stages in health and disease and will provide further insights into mechanisms of FMNT generation.

The preliminary results obtained by forced expression of Dlk1 in chick MNs indicate that Dlk1 is capable to promote a transition in some electrophysiological parameters to a faster FMNT phenotype. This will therefore open up the possibility to study the contribution of distinct Dlk1 domains or isoforms towards their specific impact on MN function in the near future. Moreover, initial results from MNs in Dlkl knockout mice suggested a more pronounced bistable behavior as well as an earlier response towards the injected current (Fig. 6.4). Although the number of cells has to be increased these findings are consistent with a shift in electrophysiogical properties towards slower characteristics.

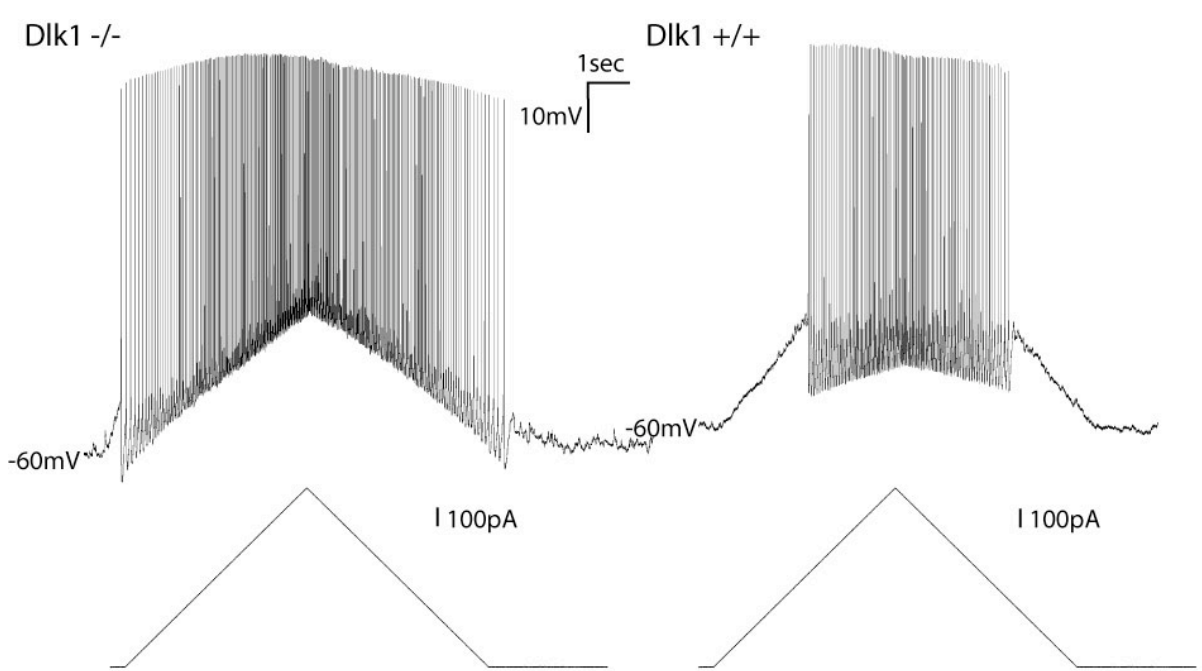

Fig. 6.4 The bistable behavior of MNs is altered in Dlk1 knockout mice Patch clamp recordings of P4 MNs revealed a varying bistable behavior of MNs in Dlk1-/mice. Also the excitability of Dlk1-/- MNs is increased, as a lower current is necessary to evoke action potentials.

If these results can be finally verified the Dlk1 gene will not only be one of the first FMNT specific marker genes but also one the first identified determinant of FMNT fate. Another aim of ongoing research is to elucidate the mechanisms of Dlk1 effects within MNs, for this purpose a screen for potential Dlk1 interaction partners on the proteome level has been prepared and will be performed in the near future to understand how Dlk1 exerts its activity in MNs. In addition also the Dlk2 knockout 


\section{Discussion}

mice will be obtained from a collaborating group in order to examine potential functionally redundant roles of Dlk1 and Dlk2 during $\mathrm{MN}$ development. Future studies will also be performed in conditional Dlk1 and PRKAG2 knockout animals, thereby it will be finally verified if the observed effects of Dlk1 are motor neuron specific and whether can be reproduced within animals lacking the putative downstream target gene PRKAG2. Also the effects of Dlk1 and PRKAG2 towards MN metabolism are going to be further investigated (Fig. 6.5). Moreover, the apparent shift from faster to slower FMNT properties obtained in $D l k 1^{-/}$animals may offer the opportunity to genetically reprogram the FMNT status to a more resistant profile in ALS transgenic mouse models. Hence, Dlk1 knockout mice were mated with SOD G93A transgenic mice to elucidate effects on the survival in this ALS animal model.

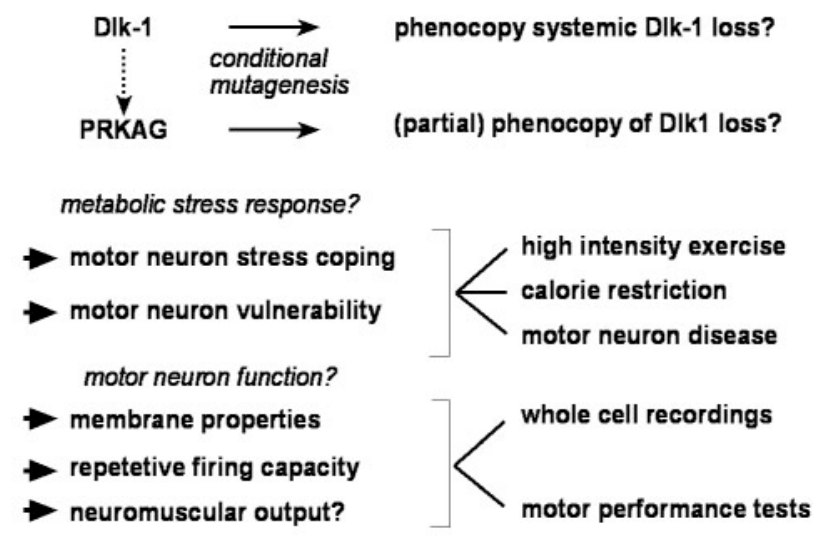

Fig. 6.5 Further studies to elucidate the role of Dlk1 in motor neuron development and energy metabolism Future studies in Dlk1 and PRKAG2 conditional knockout animals will help to examine the role of these proteins in $\mathrm{MN}$ development in general and MN energy metabolism in paricular. 


\section{Summary}

\section{Summary}

In the context of this work, two different populations of motor neurons have been determined based on the muscle fiber composition of the innervated muscles the fast muscle fiber enriched Rectus femoris and the slow muscle fiber enriched Soleus. These populations have been analyzed in terms of their differential gene expression in order to identify molecular markers of different functional motor neuron types.

In this work it was possible to identify the expression of the $D l k 1$ gene as a marker for fast motor neurons. The expression of the $D l k l$ gene was assed in different embryonic and postnatal stages in mice revealing its presence from early postmitotic motor neurons at E12.5 up to almost mature P10 motor neurons. This provided unanticipated evidence that the differentiation of fast and slow FMNTs may already have occurred prior to muscle innervation.

During this work, Dlkl deficient mice were examined on a behavioral and at the molecular level. While the behavioral experiments have revealed general deficits in overall neuromuscular performance in Dlkl knockout animals, the studies of Dlk1 effects on motor neuron gene expression have shown alterations in a set of genes involved in the cellular energy metabolism as well as in inflammation. Intriguingly, this was further accompanied by a switch in the expression of a defined set of genes from fast to slow MNs. A central component of the genes affected by Dlk1 was the AMPK gamma subunit 2, suggesting a central role of Dlk1 in the regulation of the MNs energy metabolism.

Subsequent electrophysiological experiments in chicken embryo motor neurons have shown alterations in properties associated with functional motor neuron types upon Dlk1 overexpression. These preliminary observations suggest Dlk1 to be sufficient to drive motor neuron specification towards fast motor neuron properties, while being required for the acquisition of specific fast $\mathrm{MN}$ properties.

Thus, this thesis project revealed the first evidence for an early molecular marker involved in the specification of fast and slow FMNTs. 


\section{Literature}

\section{Literature}

Abramoff MD., Magelhaes PJ. \& Ram SJ. (2004) Image Processing with ImageJ Biophotonics International, volume 11, issue 7, pp. 36-42

Agalliu D., Takada S., Agalliu I., McMahon AP. \& Jessell TM. (2009) Motor neurons with axial muscle projections specified by Wnt4/5 signaling. Neuron. Mar 12;61(5):708-20.

Astuti D., Latif F., Wagner K., Gentle D., Cooper WN., Catchpoole D., Grundy R., Ferguson-Smith AC. \& Maher ER. (2005) Epigenetic alteration at the DLK1GTL2 imprinted domain in human neoplasia: analysis of neuroblastoma, phaeochromocytoma and Wilms' tumour. Br J Cancer 92: 1574-1580

Bacou F., Rouanet P., Barjot C., Janmot C., Vigneron P. \& d'Albis A. (1996) Expression of myosin isoforms in denervated, cross-reinnervated, and electrically stimulated rabbit muscles. Eur J Biochem. Mar 1;236(2):539-47.

Bakels R. \& Kernell D. (1993) Matching between motoneurone and muscle unit properties in rat medial gastrocnemius. J Physiol. Apr;463:307-24.

Baladrón V., Ruiz-Hidalgo MJ., Nueda ML., Díaz-Guerra MJ., García-Ramírez JJ., Bonvini E., Gubina E. \& Laborda J. (2005) dlk acts as a negative regulator of Notch1 activation through interactions with specific EGF-like repeats. Exp Cell Res. Feb 15;303(2):343-59.

Banks RW. (1994) The motor innervation of mammalian muscle spindles. Prog Neurobiol. Jul-Aug;43(4-5):323-62. 


\section{Literature}

Bauer M., Szulc J., Meyer M., Jensen CH., Terki TA., Meixner A., Kinkl N., Gasser T., Aebischer P. \& Ueffing M. (2008) Delta-like 1 participates in the specification of ventral midbrain progenitor derived dopaminergic neurons. $\mathrm{J}$ Neurochem. Feb;104(4):1101-15.

Betley JN., Wright CV., Kawaguchi Y., Erdélyi F., Szabó G., Jessell TM. \& Kaltschmidt JA. (2009) Stringent specificity in the construction of a GABAergic presynaptic inhibitory circuit. Cell. Oct 2;139(1):161-74.

Bettenhausen B., Hrabĕ de Angelis M., Simon D., Guénet JL. \& Gossler A. (1995) Transient and restricted expression during mouse embryogenesis of Dll1, a murine gene closely related to Drosophila Delta. Development. Aug;121(8):2407-18.

Bowden RE. \& Mahran ZY. (1956) The functional significance of the pattern of innervation of the muscle quadratus labii superioris of the rabbit, cat and rat. J Anat. Apr;90(2):217-27.

Bray SJ., Takada S., Harrison E., Shen SC. \& Ferguson-Smith AC. (2008) The atypical mammalian ligand Delta-like homologue 1 (Dlk1) can regulate Notch signalling in Drosophila. BMC Dev Biol. Jan 31;8:11.

Briscoe J. \& Ericson J. (2001) Specification of neuronal fates in the ventral neural tube. Curr Opin Neurobiol. Feb;11(1):43-9.

Briscoe J., Pierani A., Jessell TM. \& Ericson J. (2000) A homeodomain protein code specifies progenitor cell identity and neuronal fate in the ventral neural tube. Cell. May 12;101(4):435-45.

Bruestle DA., Cutler RG., Telljohann RS. \& Mattson MP. (2009) Decline in daily running distance presages disease onset in a mouse model of ALS. Neuromolecular Med. 11(2):58-62. 


\section{Literature}

Burke RE., Strick PL., Kanda K., Kim CC. \& Walmsley B. (1977) Anatomy of medial gastrocnemius and soleus motor nuclei in cat spinal cord. J Neurophysiol. May;40(3):667-80.

Burke RE. \& Tsairis P. (1973) Anatomy and innervation ratios in motor units of cat gastrocnemius. J Physiol. Nov;234(3):749-65.

Burkholder TJ., Fingado B., Baron S. \& Lieber RL. (1994) Relationship between muscle fiber types and sizes and muscle architectural properties in the mouse hindlimb. J Morphol. Aug;221(2):177-90.

Cai J., Xu X., Yin H., Wu R., Modderman G., Chen Y., Jensen J., Hui CC. \& Qiu M. (2000) Evidence for the differential regulation of Nkx-6.1 expression in the ventral spinal cord and foregut by Shh-dependent and -independent mechanisms. Genesis. May;27(1):6-11.

Carvalho M., Schwartz MS. \& Swash M. (1995) Involvement of the external anal sphincter in amyotrophic lateral sclerosis. Muscle Nerve. Aug;18(8):848-53.

Ceder JA., Jansson L., Helczynski L. \& Abrahamsson PA. (2008) Delta-like 1 (Dlk-1), a novel marker of prostate basal and candidate epithelial stem cells, is downregulated by notch signalling in intermediate/transit amplifying cells of the human prostate. Eur Urol. Dec;54(6):1344-53.

Chacón MR., Miranda M., Jensen CH., Fernández-Real JM., Vilarrasa N., Gutiérrez C., Näf S., Gomez JM. \& Vendrell J. (2008) Human serum levels of fetal antigen 1 (FA1/Dlk1) increase with obesity, are negatively associated with insulin sensitivity and modulate inflammation in vitro. Int J Obes (Lond). Jul;32(7):1122-9.

Chakkalakal JV., Nishimune H., Ruas JL., Spiegelman BM. \& Sanes JR. (2010) Retrograde influence of muscle fibers on their innervation revealed by a novel marker for slow motoneurons. Development. Oct;137(20):3489-99. 


\section{Literature}

Chalfie M., Tu Y., Euskirchen G., Ward WW. \& Prasher DC. (1994) Green fluorescent protein as a marker for gene expression. Science. Feb 11;263(5148):8025.

Chapman G., Sparrow DB., Kremmer E. \& Dunwoodie SL. (2010) Notch inhibition by the ligand Delta-Like 3 defines the mechanism of abnormal vertebral segmentation in spondylocostal dysostosis. Hum Mol Genet. Dec 21.

Chen JC., Mortimer J., Marley J. \& Goldhamer DJ. (2005) MyoD-cre transgenic mice: a model for conditional mutagenesis and lineage tracing of skeletal muscle. Genesis. Mar;41(3):116-21.

Chiò A., Benzi G., Dossena M., Mutani R. \& Mora G. (2005) Severely increased risk of amyotrophic lateral sclerosis among Italian professional football players. Brain. Mar;128(Pt 3):472-6.

Chiò A., Mora G., Leone M., Mazzini L., Cocito D., Giordana MT., Bottacchi E. \& Mutani R. (2002) Early symptom progression rate is related to ALS outcome: a prospective population-based study. Neurology Jul 9;59(1):99-103.

Chitnis A., Henrique D., Lewis J., Ish-Horowicz D. \& Kintner C. (1995) Primary neurogenesis in Xenopus embryos regulated by a homologue of the Drosophila neurogenic gene Delta. Nature 375, 761-766.

Cockett NE., Jackson SP., Shay TL., Farnir F., Berghmans S., Snowder GD., Nielsen DM. \& Georges M. (1996) Polar overdominance at the ovine callipyge locus. Science. Jul 12;273(5272):236-8.

Dalla Torre di Sanguinetto SA., Dasen JS. \& Arber S. (2008) Transcriptional mechanisms controlling motor neuron diversity and connectivity. Curr Opin Neurobiol. Feb;18(1):36-43. 


\section{Literature}

Dasen JS., De Camilli A., Wang B., Tucker PW. \& Jessell TM. (2008) Hox repertoires for motor neuron diversity and connectivity gated by a single accessory factor, FoxP1 Cell. Jul 25;134(2):304-16.

Dasen JS. \& Jessell TM. (2009) Hox networks and the origins of motor neuron diversity. Curr Top Dev Biol.;88:169-200.

Dasen JS., Liu JP. \& Jessell TM. (2003) Motor neuron columnar fate imposed by sequential phases of Hox-c activity. Nature. Oct 30;425(6961):926-33.

Dasen JS., Tice BC., Brenner-Morton S. \& Jessell TM. (2005) A Hox regulatory network establishes motor neuron pool identity and target-muscle connectivity. Cell. Nov 4;123(3):477-91.

Davis E., Jensen CH., Schroder HD., Farnir F., Shay-Hadfield T., Kliem A., Cockett N., Georges M. \& Charlier C. (2004) Ectopic expression of DLK1 protein in skeletal muscle of padumnal heterozygotes causes the callipyge phenotype. Curr Biol. Oct 26;14(20):1858-62.

Dengler R., Konstanzer A., Küther G., Hesse S., Wolf W. \& Struppler A. (1990) Amyotrophic lateral sclerosis: macro-EMG and twitch forces of single motor units. Muscle Nerve. Jun;13(6):545-50.

Enjin A., Rabe N., Nakanishi ST., Vallstedt A., Gezelius H., Memic F., Lind M., Hjalt T., Tourtellotte WG., Bruder C., Eichele G., Whelan PJ. \& Kullander K. (2010) Identification of novel spinal cholinergic genetic subtypes disclose Chodl and Pitx2 as markers for fast motor neurons and partition cells. J Comp Neurol. Jun $15 ; 518(12): 2284-304$.

Ericson J., Rashbass P., Schedl A., Brenner-Morton S., Kawakami A., van Heyningen V., Jessell T.M. \& Briscoe J. (1997) Pax6 controls progenitor cell identity and neuronal fate in response to graded Shh signalling. Cell Vol. 90, 169-180 


\section{Literature}

Espina AG., Mendez-Vidal C., Moreno-Mateos MA., Saez C., Romero-Franco A., Japón MA. \& Pintor-Toro JA. (2009) Induction of Dlk1 by PTTG1 inhibits adipocyte differentiation and correlates with malignant transformation. Mol Biol Cell 20: $3353-3362$.

Evans AM., Hardie DG., Peers C., Wyatt CN., Viollet B., Kumar P., Dallas ML, Ross F., Ikematsu N., Jordan HL., Barr BL., Rafferty JN. \& Ogunbayo O. (2009) Ion channel regulation by AMPK: the route of hypoxia-response coupling in the carotid body and pulmonary artery. Ann N Y Acad Sci. Oct;1177:89-100.

Fay TN., Jacobs I., Teisner B., Poulsen O., Chapman MG., Stabile I., Bohn H., Westergaard JG. \& Grudzinskas JG. (1988) Two fetal antigens (FA-1 and FA-2) and endometrial proteins (PP12 and PP14) isolated from amniotic fluid; preliminary observations in fetal and maternal tissues. Eur J Obstet Gynecol Reprod Biol. Sep; 29(1):73-85.

Fahrenkrug SC., Freking BA., Rexroad CE. 3rd, Leymaster KA., Kappes SM. \& Smith TP. (2000) Comparative mapping of the ovine clpg locus. Mamm Genome. Oct;11(10):871-6.

Falk MJ, Curtis CA, Bass NE, Zinn AB, Schwartz S. (2005) Maternal uniparental disomy chromosome 14: case report and literature review. Pediatr Neurol. Feb;32(2):116-20.

Fetcho JR. (1987) A review of the organization and evolution of motoneurons innervating the axial musculature of vertebrates. Brain Res. Jul;434(3):243-80.

Floridon C., Jensen CH., Thorsen P., Nielsen O., Sunde L., Westergaard JG., Thomsen SG. \& Teisner B. (2000) Does fetal antigen 1 (FA1) identify cells with regenerative, endocrine and neuroendocrine potentials? A study of FA1 in embryonic, fetal, and placental tissue and in maternal circulation. Differentiation. Aug;66(1):4959. 


\section{Literature}

Frey D., Schneider C., Xu L., Borg J., Spooren W. \& Caroni P. (2000) Early and selective loss of neuromuscular synapse subtypes with low sprouting competence in motoneuron diseases. J Neurosci. Apr 1;20(7):2534-42.

Friese A., Kaltschmidt JA., Ladle DR., Sigrist M., Jessell TM. \& Arber S. (2009) Gamma and alpha motor neurons distinguished by expression of transcription factor Err3. Proc Natl Acad Sci U S A. Aug 11;106(32):13588-93.

Folmes KD., Chan AY., Koonen DP., Pulinilkunnil TC., Baczkó I., Hunter BE., Thorn S., Allard MF., Roberts R., Gollob MH., Light PE. \& Dyck JR. (2009) Distinct early signaling events resulting from the expression of the PRKAG2 R302Q mutant of AMPK contribute to increased myocardial glycogen. Circ Cardiovasc Genet. Oct;2(5):457-66.

Fournier le Ray C., Renaud D. \& Le Douarin GH. (1989) Change in motor neurone activity modifies the differentiation of a slow muscle in chick embryo. Development Jun;106(2):295-302.

Fuentes I., Cobos AR. \& Segade LA. (1998) Muscle fibre types and their distribution in the biceps and triceps brachii of the rat and rabbit. J Anat. Feb;192 ( Pt 2):203-10.

Fujinaga Y., Wolf AA., Rodighiero C., Wheeler H., Tsai B., Allen L., Jobling MG., Rapoport T., Holmes RK. \& Lencer WI. (2003) Gangliosides that associate with lipid rafts mediate transport of cholera and related toxins from the plasma membrane to endoplasmic reticulm. Mol Biol Cell. Dec;14(12):4783-93.

Gardiner PF. (1993) Physiological properties of motoneurons innervating different muscle unit types in rat gastrocnemius. J Neurophysiol. Apr;69(4):1160-70.

Gizzi M., DiRocco A., Sivak M. \& Cohen B. (1992) Ocular motor function in motor neuron disease. Neurology. May;42(5):1037-46. 


\section{Literature}

Gollob MH., Green MS., Tang AS., Gollob T., Karibe A., Ali Hassan AS., Ahmad F., Lozado R., Shah G., Fananapazir L., Bachinski LL. \& Roberts R. (2001) Identification of a gene responsible for familial Wolff-Parkinson-White syndrome. N Engl J Med. Jun 14;344(24):1823-31.

Gordon WR., Arnett KL. \& Blacklow SC. (2008) The molecular logic of Notch signaling--a structural and biochemical perspective. J Cell Sci. Oct 1;121(Pt 19):310919.

Green J., Erdmann G. \& Wellhöner HH. (1977) Is there retrograde axonal transport of tetanus toxin in both alpha and gamma fibres? Nature. Jan 27;265(5592):370.

Gu H., Zou YR. \& Rajewsky K. (1993) Independent control of immunoglobulin switch recombination at individual switch regions evidenced through Cre-loxPmediated gene targeting. Cell. 73:1155-1164

Gutman CR., Ajmera MK. \& Hollyday, M. (1993) Organization of motor pools supplying axial muscles in the chicken. Brain Res. 609, 129-136.

Gubina E., Ruiz-Hidalgo MJ., Baladrón V. \& Laborda J. (1999) Assignment of DLK1 to human chromosome band $14 \mathrm{q} 32$ by in situ hybridization. Cytogenet Cell Genet. 84(3-4):206-7.

Gubina E., Ruiz-Hidalgo MJ., Baladrón V. \& Laborda J. (2000) Assignment of dlk (Dlk1) to mouse chromosome band 12E-F1 by in situ hybridization. Cytogenet Cell Genet. 88(3-4):322-3.

Gurney ME., Pu H., Chiu AY., Dal Canto MC., Polchow CY., Alexander DD., Caliendo J., Hentati A., Kwon YW., Deng HX. et al. (1994) Motor neuron degeneration in mice that express a human $\mathrm{Cu}, \mathrm{Zn}$ superoxide dismutase mutation. Science. Jun 17;264(5166):1772-5. 


\section{Literature}

Guy J., Hendrich B., Holmes M., Martin JE. \& Bird A. (2001) A mouse Mecp2null mutation causes neurological symptoms that mimic Rett syndrome. Nat Genet. Mar;27(3):322-6.

Hall TA. (1999) BioEdit: a user-friendly biological sequence alignment editor and analysis program for Windows 95/98/NT. Nucl. Acids. Symp. Ser. 41:95-98.

Hansson O., Nylandsted J., Castilho RF., Leist M., Jäättelä M. \& Brundin P. (2003) Overexpression of heat shock protein 70 in R6/2 Huntington's disease mice has only modest effects on disease progression. Brain Res. Apr 25;970(1-2):47-57.

Harburger DS. \& Calderwood DA. (2009) Integrin signalling at a glance. J Cell Sci. Jan 15;122(Pt 2):159-63.

Hashizume K., Kanda K. \& Burke RE. (1988) Medial gastrocnemius motor nucleus in the rat: age-related changes in the number and size of motoneurons. J Comp Neurol. Mar 15;269(3):425-30.

Hegedus J., Putman CT. \& Gordon T. (2007) Time course of preferential motor unit loss in the SOD1 G93A mouse model of amyotrophic lateral sclerosis. Neurobiol Dis. Nov;28(2):154-64.

Henrique D., Adam J., Myat A., Chitnis A., Lewis J. \& Ish-Horowicz D. (1995) Expression of a Delta homologue in prospective neurons in the chick. Nature 375, 787-790.

Henriques A., Pitzer C. \& Schneider A. (2010) Characterization of a novel SOD1(G93A) transgenic mouse line with very decelerated disease development. PLoS One. Nov 11;5(11):e15445.

Hoess RH., Ziese M. \& Sternberg N. (1982) P1 site-specific recombination: nucleotide sequence of the recombining sites. Proc Natl Acad Sci U S A. Jun;79(11):3398-402. 


\section{Literature}

Hollyday M. \& Hamburger V. (1997) An autoradiographic study of the formation of the lateral motor column in the chick embryo. Brain Res. Aug 26;132(2):197-208.

Honig MG., Frase PA. \& Camilli SJ. (1998) The spatial relationships among cutaneous, muscle sensory and motoneuron axons during development of the chick hindlimb. Development. Mar;125(6):995-1004.

Honig MG. \& Hume RI. (1986) Fluorescent carbocyanine dyes allow living neurons of identified origin to be studied in long-term cultures. J Cell Biol. Jul;103(1):171-87.

Honig MG. \& Hume RI. (1989) Dil and DiO: versatile fluorescent dyes for neuronal labelling and pathway tracing. Trends Neurosci. Sep;12(9):333-5, 340-1.

Hunt CC. \& Kuffler SW. (1951) Further study of efferent small-nerve fibers to mammalian muscle spindles; multiple spindle innervation and activity during contraction. J Physiol. Apr;113(2-3):283-97.

Inoue H., Nojima H. \& Okayama H. (1990) High efficiency transformation of Escherichia coli with plasmids. Gene. Nov 30;96(1):23-8.

Ishihara A., Naitoh H., Araki H. \& Nishihira Y. (1988) Soma size and oxidative enzyme activity of motoneurones supplying the fast twitch and slow twitch muscles in the rat. Brain Res. Apr 12;446(1):195-8.

Ivanova A., Signore M., Caro N., Greene ND., Copp AJ. \& Martinez-Barbera JP. (2005) In vivo genetic ablation by Cre-mediated expression of diphtheria toxin fragment A. Genesis. Nov;43(3):129-35.

Jansson E. \& Sylvén C. (1983) Myoglobin concentration in single type I and type II muscle fibres in man. Histochemistry. 78(1):121-4.

Jensen, CH., Meyer M., Schroder HD., Kliem A., Zimmer J. \& Teisner B. (2001) Neurons in the monoaminergic nuclei of the rat and human central nervous system express FA1/dlk. NeuroReport 12, 3959-3963. 


\section{Literature}

Jessell TM. (2000) Neuronal specification in the spinal cord: inductive signals and transcriptional codes. Nat. Rev. Genet. Oct;1(1):20-9.

Kaminski HJ., Richmonds CR., Kusner LL. \& Mitsumoto H. (2002) Differential susceptibility of the ocular motor system to disease. Ann N Y Acad Sci. Apr; 956:4254.

Kaneta M., Osawa M., Sudo K., Nakauchi H., Farr AG. \& Takahama Y. (2000) A role for pref-1 and HES-1 in thymocyte development. J Immunol. Jan $1 ; 164(1): 256-64$.

Kanning KC., Kaplan A. \& Henderson CE. (2010) Motor neuron diversity in development and disease. Annu Rev Neurosci. 33:409-40.

Kato K., Shimizu A. \& Totsuka T. (1988) Developmental changes in fibre type-type related proteins in soleus, rectus femoris and heart muscles of normal and dystrophic mice. J. Neurol. Sci. Jun; 85(2): 161-171.

Kawakami K. \& Shima A. (1999) Identification of the Tol2 transposase of the medaka fish Oryzias latipes that catalyzes excision of a nonautonomous Tol 2 element in zebrafish Danio rerio. Gene 240, 239-244

Kawakami T., Chano T., Minami K., Okabe H., Okada Y. \& Okamoto K. (2006) Imprinted DLK1 is a putative tumor suppressor gene and inactivated by epimutation at the region upstream of GTL2 in human renal cell carcinoma. Hum Mol Genet 15: $821-830$.

Kemm RE. \& Westbury DR. (1978) Some properties of spinal gammamotoneurones in the cat, determined by micro-electrode recording. J Physiol. Sep;282:59-71. 


\section{Literature}

Kemp BE., Mitchelhill KI., Stapleton D., Michell BJ., Chen ZP. \& Witters LA. (1999) Dealing with energy demand: the AMP-activated protein kinase. Trends Biochem Sci. Jan;24(1):22-5.

Kernell D. (2003) Principles of force gradation in skeletal muscles. Neural Plast. 10(1-2):69-76.

Kernell D. \& Zwaagstra B. (1981) Input conductance axonal conduction velocity and cell size among hindlimb motoneurones of the cat. Brain Res. Jan 12;204(2):31126.

Kim KA., Kim JH., Wang Y. \& Sul HS. (2007) Pref-1 (preadipocyte factor 1) activates the MEK/extracellular signal-regulated kinase pathway to inhibit adipocyte differentiation. Mol Cell Biol. Mar;27(6):2294-308.

Kojima H., Furuta Y., Fujita M., Fujioka Y. \& Nagashima K. (1989) Onuf's motoneuron is resistant to poliovirus. J Neurol Sci. Oct;93(1):85-92.

Komatsu H., Chao MY., Larkins-Ford J., Corkins ME., Somers GA., Tucey T., Dionne HM., White JQ., Wani K., Boxem M. \& Hart AC. (2008) OSM-11 facilitates LIN-12 Notch signaling during Caenorhabditis elegans vulval development. PLoS Biol. Aug 12;6(8):e196.

Kréneisz O., Benoit JP., Bayliss DA. \& Mulkey DK. (2009) AMP-activated protein kinase inhibits TREK channels. J Physiol. Dec 15;587(Pt 24):5819-30.

Kristensson K. \& Olsson Y. (1971) Retrograde axonal transport of protein. Brain Res. Jun 18;29(2):363-5.

Kuffler SW., Hunt CC. \& Quilliam P. (1951) Function of medullated small-nerve fibers in mammalian ventral roots; efferent muscle spindle innervation. J Neurophysiol. Jan;14(1):29-54. 


\section{Literature}

Kwiatkowski TJ. Jr., Bosco DA., Leclerc AL., Tamrazian E., Vanderburg CR., Russ C., Davis A., Gilchrist J., Kasarskis EJ., Munsat T., Valdmanis P., Rouleau GA., Hosler BA., Cortelli P., de Jong PJ., Yoshinaga Y., Haines JL., PericakVance MA., Yan J., Ticozzi N., Siddique T., McKenna-Yasek D., Sapp PC., Horvitz HR., Landers JE. \& Brown RH. Jr. (2009) Mutations in the FUS/TLS gene on chromosome 16 cause familial amyotrophic lateral sclerosis. Science. Feb 27;323(5918):1205-8.

Laborda J., Sausville EA., Hoffman T. \& Notario V. (1993) dlk, a putative mammalian homeotic gene differentially expressed in small cell lung carcinoma and neuroendocrine tumor cell line. J Biol Chem. Feb 25;268(6):3817-20.

Landmesser L. (1978) The distribution of motoneurones supplying chick hind limb muscles. J Physiol. Nov;284:371-89.

Lang UE., Puls I., Muller DJ., Strutz-Seebohm N. \& Gallinat J. (2007) Molecular mechanisms of schizophrenia. Cell Physiol Biochem. 20(6):687-702.

Larsson L., Edström L., Lindegren B., Gorza L. \& Schiaffino S. (1991) MHC composition and enzyme-histochemical and physiological properties of a novel fasttwitch motor unit type. Am J Physiol. Jul;261(1 Pt 1):C93-101.

Lee K., Villena JA., Moon YS., Kim KH., Lee S., Kang C. \& Sul HS. (2003) Inhibition of adipogenesis and development of glucose intolerance by soluble preadipocyte factor-1 (Pref-1). J Clin Invest. Feb;111(4):453-61.

Lee SK., Jurata LW., Funahashi J., Ruiz EC. \& Pfaff SL. (2004) Analysis of embryonic motoneuron gene regulation: derepression of general activators function in concert with enhancer factors. Development. Jul;131(14):3295-306.

Lee SK., Lee B., Ruiz EC. \& Pfaff SL. (2005) Olig2 and Ngn2 function in opposition to modulate gene expression in motor neuron progenitor cells. Genes Dev. Jan 15;19(2):282-94. 
Lein ES., Hawrylycz MJ., Ao N., Ayres M., Bensinger A., Bernard A., Boe AF., Boguski MS., Brockway KS., Byrnes EJ., Chen L., Chen L., Chen TM., Chin MC., Chong J., Crook BE., Czaplinska A., Dang CN., Datta S., Dee NR., Desaki AL., Desta T., Diep E., Dolbeare TA., Donelan MJ., Dong HW., Dougherty JG., Duncan BJ., Ebbert AJ., Eichele G., Estin LK., Faber C., Facer BA., Fields R., Fischer SR., Fliss TP., Frensley C., Gates SN., Glattfelder KJ., Halverson KR., Hart MR., Hohmann JG., Howell MP., Jeung DP., Johnson RA., Karr PT., Kawal R., Kidney JM., Knapik RH., Kuan CL., Lake JH., Laramee AR., Larsen KD., Lau C., Lemon TA., Liang AJ., Liu Y., Luong LT., Michaels J., Morgan JJ., Morgan RJ., Mortrud MT., Mosqueda NF., Ng LL., Ng R., Orta GJ., Overly CC., Pak TH., Parry SE., Pathak SD., Pearson OC., Puchalski RB., Riley ZL., Rockett HR., Rowland SA., Royall JJ., Ruiz MJ., Sarno NR., Schaffnit K., Shapovalova NV., Sivisay T., Slaughterbeck CR., Smith SC., Smith KA., Smith BI., Sodt AJ., Stewart NN., Stumpf KR., Sunkin SM., Sutram M., Tam A., Teemer CD., Thaller C., Thompson CL., Varnam LR., Visel A., Whitlock RM., Wohnoutka PE., Wolkey CK., Wong VY., Wood M., Yaylaoglu MB., Young RC., Youngstrom BL., Yuan XF., Zhang B., Zwingman TA. \& Jones AR. (2007) Genome-wide atlas of gene expression in the adult mouse brain. Nature. Jan $11 ; 445(7124): 168-76$.

Li S., Czubryt MP., McAnally J., Bassel-Duby R., Richardson JA., Wiebel FF., Nordheim A. \& Olson EN. (2005) Requirement for serum response factor for skeletal muscle growth and maturation revealed by tissue-specific gene deletion in mice. Proc Natl Acad Sci U S A. Jan 25;102(4):1082-7.

Li Z. \& Murthy VN. (2001) Visualizing postendocytic traffic of synaptic vesicles at hippocampal synapses. Neuron. Aug 30;31(4):593-605.

Licastro F., Chiappelli M., Grimaldi LM., Morgan K., Kalsheker N., Calabrese E., Ritchie A., Porcellini E., Salani G., Franceschi M. \& Canal N. (2005) A new promoter polymorphism in the alpha-1-antichymotrypsin gene is a disease modifier of Alzheimer's disease. Neurobiol Aging. Apr;26(4):449-53. 


\section{Literature}

Light PE., Wallace CH. \& Dyck JR. (2003) Constitutively active adenosine monophosphate-activated protein kinase regulates voltage-gated sodium channels in ventricular myocytes. Circulation. Apr 22;107(15):1962-5.

Lim GP., Backstrom JR., Cullen MJ., Miller CA., Atkinson RD. \& Tökés ZA. (1996) Matrix metalloproteinases in the neocortex and spinal cord of amyotrophic lateral sclerosis patients. J Neurochem. Jul;67(1):251-9.

Lin S., Landmann L., Ruegg MA. \& Brenner HR. (2008) The role of nerve- versus muscle-derived factors in mammalian neuromuscular junction formation. J Neurosci. Mar 26;28(13):3333-40.

Lu BD., Allen DL., Leinwand LA. \& Lyons GE. (1999) Spatial and temporal changes in myosin heavy chain gene expression in skeletal muscle development. Dev. Biol. Dec 1;216(1):312-326.

Lu QR., Sun T., Zhu Z., Ma N., Garcia M., Stiles CD. \& Rowitch DH. (2002) Common developmental requirement for Olig function indicates a motor neuron/oligodendrocyte connection. Cell. Apr 5;109(1):75-86.

Mangiarini L., Sathasivam K., Seller M., Cozens B., Harper A., Hetherington C., Lawton M., Trottier Y., Lehrach H., Davies SW. \& Bates GP. (1996) Exon 1 of the HD gene with an expanded CAG repeat is sufficient to cause a progressive neurological phenotype in transgenic mice. Cell. Nov 1;87(3):493-506.

Mannen T. (2000) Neuropathological findings of Onuf's nucleus and its significance. Neuropathology. Sep;20 Suppl:S30-3.

Mantilla CB. \& Sieck G.C. (2003) Mechanisms underlying motor unit plasticity in the respiratory system. J. Appl. Physiol. Mar;94(3):1230-41.

Martí E., Bumcrot DA., Takada R. \& McMahon AP. (1995) Requirement of 19K form of Sonic hedgehog for induction of distinct ventral cell types in CNS explants. Nature. May 25;375(6529):322-5. 


\section{Literature}

Matsuda T. \& Cepko CL. (2004) Electroporation and RNA interference in the rodent retina in vivo and in vitro. Proc Natl Acad Sci U S A. Jan 6;101(1):16-22.

Matsumoto A., Nagatomo F., Mori A, Ohira Y. \& Ishihara A. (2007) Cell size and oxidative enzyme activity of rat biceps brachii and triceps brachii muscles. J Physiol Sci. Oct;57(5):311-6.

Mei B., Zhao L., Chen L. \& Sul HS. (2002) Only the large soluble form of preadipocyte factor-1 (Pref-1), but not the small soluble and membrane forms, inhibits adipocyte differentiation: role of alternative splicing. Biochem J. May 15;364(Pt $1): 137-44$

Moon YS., Smas CM., Lee K., Villena JA., Kim KH., Yun EJ. \& Sul HS. (2002) Mice lacking paternally expressed Pref-1/Dlk1 display growth retardation and accelerated adiposity. Mol Cell Biol. Aug;22(15):5585-92.

Murphy SK., Freking BA., Smith TP., Leymaster K., Nolan CM., Wylie AA., Evans HK. \& Jirtle RL. (2005) Abnormal postnatal maintenance of elevated DLK1 transcript levels in callipyge sheep. Mamm Genome. Mar;16(3):171-83.

Novitch BG., Chen AI. \& Jessell TM. (2001) Coordinate regulation of motor neuron subtype identity and pan-neuronal properties by the bHLH repressor Olig2. Neuron. Sep 13;31(5):773-89.

Nueda ML., Baladrón V., García-Ramírez JJ., Sánchez-Solana B., Ruvira MD., Rivero S., Ballesteros MA., Monsalve EM., Díaz-Guerra MJ., Ruiz-Hidalgo MJ. \& Laborda J. (2007) The novel gene EGFL9/Dlk2, highly homologous to Dlk1, functions as a modulator of adipogenesis. J Mol Biol. Apr 13;367(5):1270-80.

Nueda ML., García-Ramírez JJ., Laborda J. \& Baladrón V. (2008) dlk1 specifically interacts with insulin-like growth factor binding protein 1 to modulate adipogenesis of 3T3-L1 cells. J Mol Biol. Jun 6;379(3):428-42. 


\section{Literature}

Okada A., Lansford R., Weimann JM., Fraser SE. \& McConnell SK. (1999) Imaging cells in the developing nervous system with retrovirus expressing modified green fluorescent protein. Exp Neurol. Apr;156(2):394-406.

Okamoto M., Takemori H., Halder SK., Nonaka Y. \& Hatano O. (1998) Implication of ZOG protein (zona glomerulosa-specific protein) in zone development of the adrenal cortex. Endocr Res. Aug-Nov; 24(3-4):515-20.

Oppenheim RW. (1991) Cell death during development of the nervous system. Annu Rev Neurosci. 14:453-501.

Padmanabhan J., Levy M., Dickson DW. \& Potter H. (2006) Alpha1antichymotrypsin, an inflammatory protein overexpressed in Alzheimer's disease brain, induces tau phosphorylation in neurons. Brain. Nov;129(Pt 11):3020-34.

Pang TY., Stam NC., Nithianantharajah J., Howard ML. \& Hannan AJ. (2006) Differential effects of voluntary physical exercise on behavioral and brain-derived neurotrophic factor expression deficits in Huntington's disease transgenic mice. Neuroscience. Aug 25;141(2):569-84.

Pankov R. \& Yamada KM. (2002) Fibronectin at a glance. J Cell Sci. Oct 15;115(Pt 20):3861-3 .

Pearson JK. \& Sickles DW. (1987) Enzyme activity changes in rat soleus motoneurons and muscle after synergist ablation. J Appl Physiol. Dec;63(6):2301-8.

Peng CY, Yajima H, Burns CE, Zon LI, Sisodia SS, Pfaff SL, Sharma K. (2007) Notch and MAML signaling drives Scl-dependent interneuron diversity in the spinal cord. Neuron. Mar 15;53(6):813-27.

Pette D. \& Vrbová G. (1992) Adaptation of mammalian skeletal muscle fibers to chronic electrical stimulation. Rev Physiol Biochem Pharmacol. 120:115-202 
Pfaff SL., Mendelsohn M., Stewart CL., Edlund T. \& Jessell TM. (1996) Requirement for LIM homeobox gene Is11 in motor neuron generation reveals a motor neuron-dependent step in interneuron differentiation. Cell. Jan 26;84(2):309-20.

Pickard BS., Malloy MP., Porteous DJ., Blackwood DH. \& Muir WJ. (2005) Disruption of a brain transcription factor, NPAS3, is associated with schizophrenia and learning disability. Am J Med Genet B Neuropsychiatr Genet. Jul 5;136B(1):2632.

Price SR. \& Briscoe J. (2004) The generation and diversification of spinal motor neurons: signals and responses. Mech Dev. Sep;121(9):1103-15.

Price SR., De Marco Garcia NV., Ranscht B. \& Jessell TM. (2002) Regulation of motor neuron pool sorting by differential expression of type II cadherins. Cell. Apr 19;109(2):205-16.

Pun S., Santos AF., Saxena S., Xu L. \& Caroni P. (2006) Selective vulnerability and pruning of phasic motoneuron axons in motoneuron disease alleviated by CNTF. Nat Neurosci. Mar;9(3):408-19.

Rafuse VF., Milner LD. \& Landmesser LT. (1996) Selective innervation of fast and slow muscle regions during early chick neuromuscular development. J. Neurosci. Nov 1;16(21):6864-77.

Raghunandan R., Ruiz-Hidalgo M., Jia Y., Ettinger R., Rudikoff E., Riggins P., Farnsworth R., Tesfaye A., Laborda J. \& Bauer SR. Dlk1 influences differentiation and function of B lymphocytes. Stem Cells Dev. 2008 Jun;17(3):495507.

Rajakumar N., Elisevich K. \& Flumerfelt BA. (1993) Biotinylated dextran: a versatile anterograde and retrograde neuronal tracer. Brain Res. Apr 2;607(1-2):47-53.

Richardson WD., Smith HK., Sun T., Pringle NP., Hall A. \& Woodruff R. (2000) Oligodendrocyte lineage and the motor neuron connection. Glia. Jan 15;29(2):136-42. 


\section{Literature}

Robertson B. \& Grant G. (1985) A comparison between wheat germ agglutinin-and choleragenoid-horseradish peroxidase as anterogradely transported markers in central branches of primary sensory neurones in the rat with some observations in the cat. Neuroscience. Mar;14(3):895-905.

Romanes GJ. (1951) The motor cell columns of the lumbo-sacral spinal cord of the cat. J Comp Neurol. Apr;94(2):313-63.

Rosen DR., Siddique T., Patterson D., Figlewicz DA., Sapp P., Hentati A., Donaldson D., Goto J., O'Regan JP., Deng HX. et al. (1993) Mutations in Cu/Zn superoxide dismutase gene are associated with familial amyotrophic lateral sclerosis. Nature. Mar 4;362(6415):59-62.

Ross DA., Rao PK. \& Kadesch T. (2004) Dual roles for the Notch target gene Hes-1 in the differentiation of 3T3-L1 preadipocytes. Mol Cell Biol. Apr;24(8):3505-13.

Saiki RK., Gelfand DH., Stoffel S., Scharf SJ., Higuchi R., Horn GT., Mullis KB \& Erlich HA. (1988) Primer-directed enzymatic amplification of DNA with a thermostable DNA polymerase. Science. Jan 29;239(4839):487-91.

Sambrook J., Fritsch EF. \& Maniatis T. (1989) Molecular cloning. A Laboratory Manual $2^{\text {nd }}$ Ed. CSHL Press, Cold Spring Harbor Laboratory.

Sato N., Wang S., Li L., Okabe K., Hashimoto M., Yaginuma H., Mikoshiba K., Uchiyama Y., Uetsuki T., Yoshikawa K., Milligan CE. \& Oppenheim RW. (1998) A novel strategy for introducing exogenous bcl-2 into neuronal cells: the Cre/loxP system-mediated activation of bcl-2 for preventing programmed cell death using recombinant adenoviruses. Mol Cell Neurosci. Sep;12(1-2):65-78.

Sato Y., Kasai T., Nakagawa S., Tanabe K., Watanabe T., Kawakami K. \& Takahashi Y. (2007) Stable integration and conditional expression of electroporated transgenes in chicken embryos. Dev Biol. May 15;305(2):616-24. 


\section{Literature}

Schiaffino S., Gorza L., Sartore S., Saggin L., Ausoni S., Vianello M., Gundersen K. \& Lomo T. (1989) Three myosin heavy chain isoforms in type 2 skeletal muscle fibres. J Muscle Res Cell Motil. Jun;10(3):197-205.

Schiaffino S. \& Reggiani C. (1994) Myosin isoforms in mammalian skeletal muscle. J. Appl. Physiol. Aug; 77(2):493-501

Schmidt JV., Matteson PG., Jones BK., Guan XJ. \& Tilghman SM. (2000) The Dlk1 and Gtl2 genes are linked and reciprocally imprinted. Genes Dev. Aug $15 ; 14(16): 1997-2002$.

Shah V., Drill E. \& Lance-Jones C. (2004) Ectopic expression of Hoxd10 in thoracic spinal segments induces motoneurons with a lumbosacral molecular profile and axon projections to the limb. Dev Dyn. Sep;231(1):43-56.

Shaner NC., Campbell RE., Steinbach PA., Giepmans BN., Palmer AE. \& Tsien RY. (2004) Improved monomeric red, orange and yellow fluorescent proteins derived from Discosoma sp. red fluorescent protein. Nat Biotechnol. Dec;22(12):1567-72.

Shirasaki R. \& Pfaff SL. (2002) Transcriptional codes and the control of neuronal identity. Annu Rev Neurosci. 25:251-81.

Shneider NA., Brown MN., Smith CA., Pickel J. \& Alvarez FJ. (2009) Gamma motor neurons express distinct genetic markers at birth and require muscle spindlederived GDNF for postnatal survival. Neural Dev. Dec 2;4:42.

Sieck GC. \& Prakash YS. (1997) Morphological adaptations of neuromuscular junctions depend on fiber type. Can J Appl Physiol. Jun;22(3):197-230

Smas CM., Chen L., \& Sul HS. (1997) Cleavage of membrane-associated pref-1 generates a soluble inhibitor of adipocyte differentiation. Mol Cell Biol. Feb;17(2):977-88. 


\section{Literature}

Smas CM. \& Sul HS. (1993) Pref-1, a protein containing EGF-like repeats, inhibits adipocyte differentiation. Cell. May 21;73(4):725-34.

Snow PJ., Rose PK. \& Brown AG. (1976) Tracing axons and axon collaterals of spinal neurons using intracellular injection of horseradish peroxidase. Science. Jan 23;191(4224):312-3.

Soriano P., Montgomery C., Geske R. \& Bradley A. (1991) Targeted disruption of the c-src proto-oncogene leads to osteopetrosis in mice. Cell. Feb 22;64(4):693-702.

Sreedharan J., Blair IP., Tripathi VB., Hu X., Vance C., Rogelj B., Ackerley S., Durnall JC., Williams KL., Buratti E., Baralle F., de Belleroche J., Mitchell JD., Leigh PN., Al-Chalabi A., Miller CC., Nicholson G. \& Shaw CE. (2008) TDP-43 mutations in familial and sporadic amyotrophic lateral sclerosis. Science. Mar 21;319(5870):1668-72

Stoeckel K., Schwab M. \& Thoenen H. (1977) Role of gangliosides in the uptake and retrograde axonal transport of cholera and tetanus toxin as compared to nerve growth factor and wheat germ agglutinin. Brain Res. Aug 26;132(2):273-85.

Takahashi Y., Watanabe T., Nakagawa S., Kawakami K. \& Sato Y. (2008) Transposon-mediated stable integration and tetracycline-inducible expression of electroporated transgenes in chicken embryos. Methods Cell Biol. 87:271-80.

Tax FE., Yeargers JJ. \& Thomas JH. (1994) Sequence of C. elegans lag-2 reveals a cell-signalling domain shared with Delta and Serrate of Drosophila. Nature. Mar $10 ; 368(6467): 150-4$.

Thaler J., Harrison K., Sharma K., Lettieri K., Kehrl J. \& Pfaff SL. (1999) Active suppression of interneuron programs within developing motor neurons revealed by analysis of homeodomain factor HB9. Neuron. Aug;23(4):675-87.

Ulfhake B. \& Kellerth JO. (1982) Does alpha-motoneurone size correlate with motor unit type in cat triceps surae? Brain Res. Nov 18;251(2):201-9. 


\section{Literature}

Valdmanis PN. \& Rouleau GA. (2008) Genetics of familial amyotrophic lateral sclerosis. Neurology Jan 8;70(2):144-52.

Vallstedt A., Muhr J., Pattyn A., Pierani A., Mendelsohn M., Sander M., Jessell TM. \& Ericson J. (2001) Different levels of repressor activity assign redundant and specific roles to Nkx6 genes in motor neuron and interneuron specification. Neuron. Sep 13;31(5):743-55.

Vanderhorst VG. \& Holstege G. (1997) Organization of lumbosacral motoneuronal cell groups innervating hindlimb, pelvic floor, and axial muscles in the cat. J Comp Neurol. May 26;382(1):46-76.

Villena JA., Choi CS., Wang Y., Kim S., Hwang YJ., Kim YB., Cline G., Shulman GI. \& Sul HS. (2008) Resistance to high-fat diet-induced obesity but exacerbated insulin resistance in mice overexpressing preadipocyte factor-1 (Pref-1): a new model of partial lipodystrophy. Diabetes. Dec;57(12):3258-66.

Waddell JN., Zhang P., Wen Y., Gupta SK., Yevtodiyenko A., Schmidt JV., Bidwell CA., Kumar A. \& Kuang S. (2010) Dlk1 is necessary for proper skeletal muscle development and regeneration. PLoS One. Nov 29;5(11):e15055.

Wang Y., Kim KA., Kim JH. \& Sul HS. (2006) Pref-1, a preadipocyte secreted factor that inhibits adipogenesis J Nutr. Dec;136(12):2953-6.

Wang Y. \& Sul HS. (2006) Ectodomain shedding of preadipocyte factor 1 (Pref-1) by tumor necrosis factor alpha converting enzyme (TACE) and inhibition of adipocyte differentiation. Mol Cell Biol. Jul;26(14):5421-35.

Wang Y., Zhao L., Smas C. \& Sul HS. (2010) Pref-1 interacts with fibronectin to inhibit adipocyte differentiation. Mol Cell Biol. Jul;30(14):3480-92.

Webber CL. Jr. \& Pleschka K. (1976) Structural and functional characteristics of individual phrenic motoneurons. Pflugers Arch. Jul 30;364(2):113-21. 


\section{Literature}

Westbury DR. (1982) A comparison of the structures of alpha and gamma-spinal motoneurones of the cat. J Physiol. Apr;325:79-91.

Wichterle H., Lieberam I., Porter JA. \& Jessell TM. (2002) Directed differentiation of embryonic stem cells into motor neurons. Cell. Aug 9;110(3):38597.

Wicks P., Ganesalingham J., Collin C., Prevett M., Leigh NP. \& Al-Chalabi A. (2007) Three soccer playing friends with simultaneous amyotrophic lateral sclerosis. Amyotroph Lateral Scler. Jun;8(3):177-9.

Wigmore PM. \& Evans DJ. (2002) Molecular and cellular mechanisms involved in the generation of fiber diversity during myogenesis. Int. Rev. Cytol. 216:175-232.

Wigston DJ. \& English AW. (1992) Fiber-type proportions in mammalian soleus muscle during postnatal development. J. Neurobiol. Feb; 23(1):61-70.

White JD., Vuocolo T., McDonagh M., Grounds MD., Harper GS., Cockett NE. \& Tellam R. (2008) Analysis of the callipyge phenotype through skeletal muscle development; association of Dlk1 with muscle precursor cells. Differentiation. Mar;76(3):283-98.

Whitehead J., Keller-Peck C., Kucera J. \& Tourtellotte WG. (2005) Glial cell-line derived neurotrophic factor-dependent fusimotor neuron survival during development. Mech Dev. Jan;122(1):27-41.

Wu Y., Wang G., Scott SA. \& Capecchi MR. (2008) Hoxc10 and Hoxd10 regulate mouse columnar, divisional and motor pool identity of lumbar motoneurons. Development. Jan;135(1):171-82.

Wylie AA., Murphy SK., Orton TC. \& Jirtle RL. (2000) Novel imprinted DLK1/GTL2 domain on human chromosome 14 contains motifs that mimic those implicated in Igf2/H19 regulation. Genome Res. 10:1711-1718 


\section{Literature}

Yanai H., Nakamura K., Hijioka S., Kamei A., Ikari T., Ishikawa Y., Shinozaki E., Mizunuma N., Hatake K. \& Miyajima A. (2010) Dlk-1, a cell surface antigen on fetal hepatic stem/progenitor cells, is expressed in hepatocellular, colon, pancreas and breast carcinomas at a high frequency. J Biochem. Jul;148(1):85-92.

Yin D., Xie D., Sakajiri S., Miller CW., Zhu H., Popoviciu ML., Said JW., Black KL. \& Koeffler HP. (2006) DLK1: increased expression in gliomas and associated with oncogenic activities. Oncogene. Mar 23;25(13):1852-61.

Zajac FE. \& Faden JS. (1985) Relationship among recruitment order, axonal conduction velocity, and muscle-unit properties of type-identified motor units in cat plantaris muscle. J Neurophysiol. May;53(5):1303-22.

Zengel JE., Reid SA., Sypert GW. \& Munson JB. (1985) Membrane electrical properties and prediction of motor-unit type of medial gastrocnemius motoneurons in the cat. J Neurophysiol. May; 53(5):1323-44.

Zhou Y., Yamamoto M. \& Engel JD. (2000) GATA2 is required for the generation of V2 interneurons. Development. Sep;127(17):3829-38. 


\section{Publications}

\section{Publications}

Gallarda BW.*, Bonanomi D.*, Müller D.*, Brown A., Alaynick WA., Andrews SE., Lemke G., Pfaff SL. \& Marquardt T. (2008) Segregation of axial motor and sensory pathways via heterotypic trans-axonal signaling. Science. Apr 11;320(5873):233-6.

Execution of motor behaviors relies on circuitries effectively integrating immediate sensory feedback to efferent pathways controlling muscle activity. It remains unclear how, during neuromuscular circuit assembly, sensory and motor projections become incorporated into tightly coordinated, yet functionally separate pathways. We report that, within axial nerves, establishment of discrete afferent and efferent pathways depends on coordinate signaling between coextending sensory and motor projections. These heterotypic axon-axon interactions require motor axonal EphA3/EphA4 receptor tyrosine kinases activated by cognate sensory axonal ephrin-A ligands. Genetic elimination of trans-axonal ephrin-A --> EphA signaling in mice triggers drastic motor-sensory miswiring, culminating in functional efferents within proximal afferent pathways. Effective assembly of a key circuit underlying motor behaviors thus critically depends on trans-axonal signaling interactions resolving motor and sensory projections into discrete pathways.

Voigt A.*, Herholz D.*, Fiesel FC., Kaur K., Müller D., Karsten P., Weber SS., Kahle PJ., Marquardt T. \& Schulz JB. (2010) TDP-43-mediated neuron loss in vivo requires RNA-binding activity. PLoS One. Aug 18;5(8):e12247.

Alteration and/or mutations of the ribonucleoprotein TDP-43 have been firmly linked to human neurodegenerative diseases, including amyotrophic lateral sclerosis (ALS) and frontotemporal lobar degeneration (FTLD). The relative impacts of TDP-43 alteration, mutation, or inherent protein function on neural integrity, however, remain less clear--a situation confounded by conflicting reports based on transient and/or random-insertion transgenic expression. We therefore performed a stringent comparative investigation of impacts of these TDP-43 modifications on neural integrity in vivo. To achieve this, we systematically screened ALS/FTLD-associated and synthetic TDP-43 isoforms via same-site gene insertion and neural expression in Drosophila; followed by transposon-based motor neuron-specific transgenesis in a chick vertebrate system. Using this bi-systemic approach we uncovered a requirement of inherent TDP-43 RNA-binding function--but not ALS/FTLD-linked mutation, mislocalization, or truncation--for TDP-43-mediated neurotoxicity in vivo.

Müller D.*, Cyganek L.*, Herholz D.*, Poh CH., Lee T., Kawakami K. \& Marquardt T. Stable genetic tagging and manipulation of spinal neuron subtypes in chick (working title, in preparation)

*Those authors have contributed equally to this work 


\section{Acknowledgements}

\section{Acknowledgements}

Initially I want to thank my supervisor Dr. Till Marquardt, who had to suffer from me now for a very long time. But finally it is done now.

Also want to express my gratitude to Prof. Dr. Tomas Pieler and Prof. Dr. Andreas Wodarz who have agreed to join my thesis committee.

Also a big thank you to David Herholz for being a true friend through hard times (always remember: "There are no cats in America").

I want to thank my colleagues from the Marquardt lab especially Alexandra Klusowski for her helpful support and Chor Hoon Poh and Pitchaiah Cherukuri who have performed the electrophysiological studies in chicken embryo and mouse.

I also thank Dr. Lars Wittler and Dr. Phillip Grote from the MPI for Molecular Genetics for their help with the microarray processing and analysis.

Furthermore I thank Dr. Steve Bauer for providing the Dlk1 knockout mice and Dr. Venkatesh N. Murthy for the Synaptophysin-GFP plasmid.

Also I want to express my gratitude towards Anders Enjin who provided the in-situ hybridization protocol and the Esrrb, Calca and Chodl probes.

And last but not least I want to thank my family. 


\section{Résumé}

Name:

Given Name:

Date of birth:

Place of birth:

Nationality:

\section{School education}

$07 / 88-08 / 90$

$09 / 90-07 / 92$

$08 / 92-08 / 98$

09/98 - 06/01

\section{Higher education}

$10 / 01-11 / 06$

$07 / 05-04 / 06$

\section{Scientific career}

12/06-03/07

Since $04 / 07$
Müller

Daniel Andreas

December 16th 1981

Heilbronn

German

Ittlingen Elementary school

Bretzfeld Elementary school

Hohenlohe Gymnasium Öhringen

Agrarwissenschaftliches Gymnasium Öhringen, completed university entrance diploma with a final grade of 1.3

Studies in biology at the Georg-August University Göttingen, Final examinations passed with the grade ,sehr gut"

Diploma thesis at the department of Virology and Immunology of the German Primate Centre Goettingen on the topic: „Generierung rekombinanter Antikörper zum Nachweis des Prionproteins“, rated with the mark ,gut"

Scientific assistant at the University hospital, Göttingen

$\mathrm{PhD}$. thesis at the European Neuroscience Institute Göttingen on the topic: „Identification and characterization of the molecular mechanisms driving the specification of motor neurons" 


\section{Declaration}

\section{Declaration}

I, Daniel Müller herewith declare that I have written this thesis independently and without any aids or resources other than contemplated.

Göttingen January $31^{\text {st }} 2011$

Daniel Müller 